TELEPHONE: (510) 527-9876

FAX: (510) 527-8164

E-MAIL: MW@GEOTHERMEX.COM

\title{
ASSESSMENT OF THE STATE-OF-THE-ART \\ OF NUMERICAL SIMULATION OF \\ ENHANCED GEOTHERMAL SYSTEMS
}

\author{
for \\ U.S. Department of Energy \\ Washington, DC
}

via

Princeton Energy Resources International

Rockville, MD

by

GeothermEx, Inc.

Richmond, CA

with

Thunderhead Engineering Consultants

Manhattan, KS

and

Golder Associates

Redmond, WA

NOVEMBER 1999 


\section{DISCLAIMER}

This report was prepared as an account of work sponsored by an agency of the United States Government. Neither the United States Government nor any agency Thereof, nor any of their employees, makes any warranty, express or implied, or assumes any legal liability or responsibility for the accuracy, completeness, or usefulness of any information, apparatus, product, or process disclosed, or represents that its use would not infringe privately owned rights. Reference herein to any specific commercial product, process, or service by trade name, trademark, manufacturer, or otherwise does not necessarily constitute or imply its endorsement, recommendation, or favoring by the United States Government or any agency thereof. The views and opinions of authors expressed herein do not necessarily state or reflect those of the United States Government or any agency thereof. 


\section{DISCLAIMER}

Portions of this document may be illegible in electronic image products. Images are produced from the best available original document. 
TELEPHONE: (510) 527-9876

FAX: (510) 527-8164

E-MAIL: MW@GEOTHERMEX.COM

\section{CONTENTS}

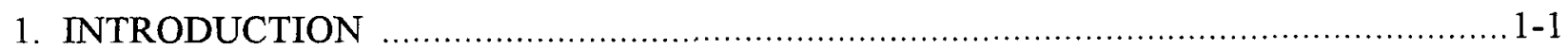

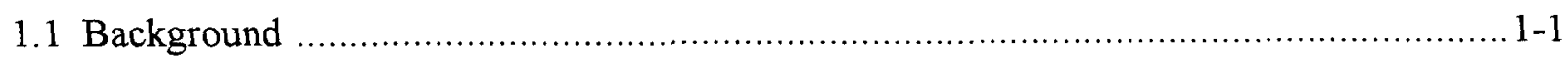

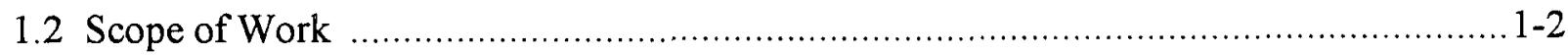

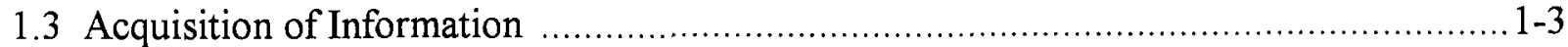

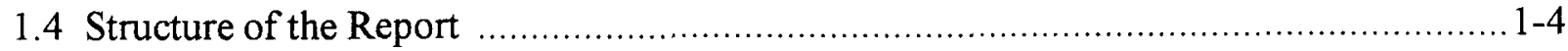

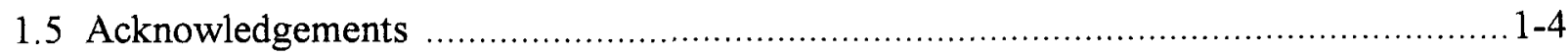

2. FEATURES OF SIMULATORS FOR ARTIFICIALLY FRACTURED SYSTEMS .........2-1

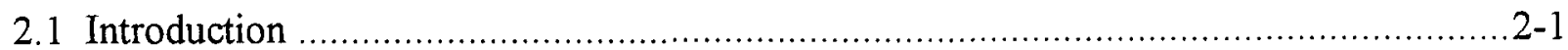

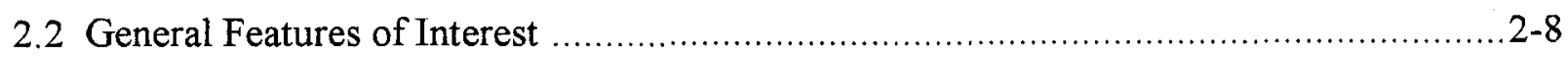

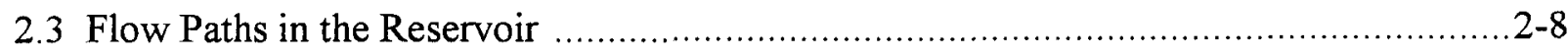

2.4 Change in Fracture Aperture Due to Effective Stress and Shear ...............................2-9

2.5 Flow Regime in the Fractures ..............................................................................

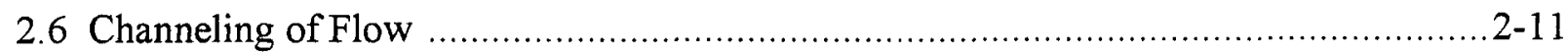

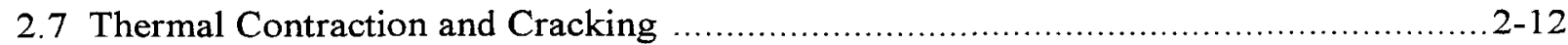

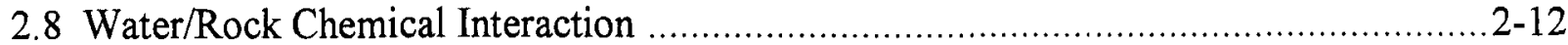

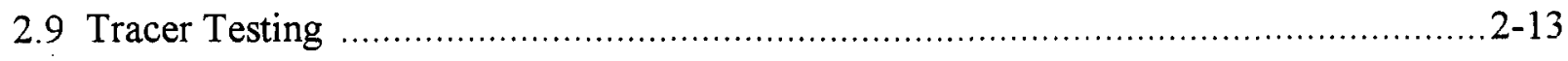

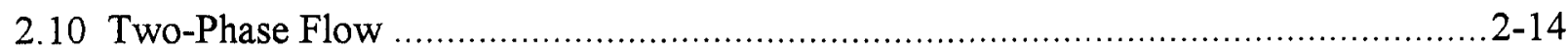

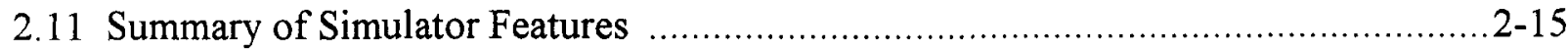

3. HDR GEOTHERMAL RESERVOIR SIMULATORS ….............................................

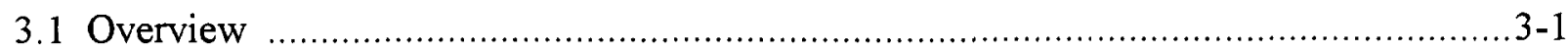

3.2 FRACTure

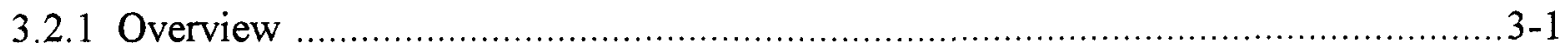

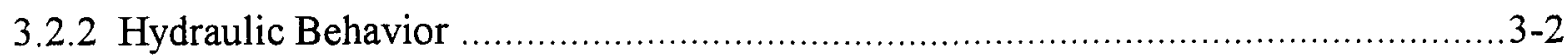


E-MAIL:MW@GEOTHERMEX.COM

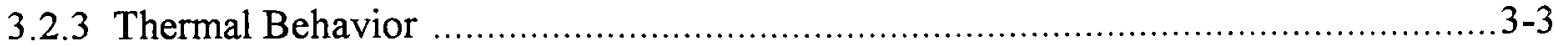

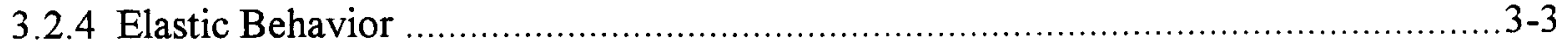

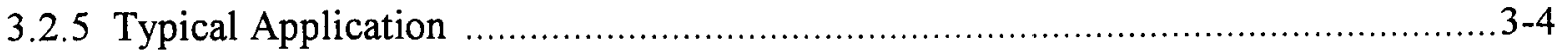

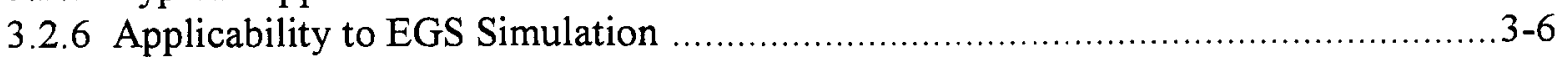

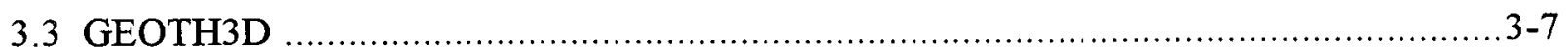

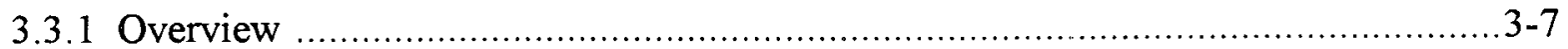

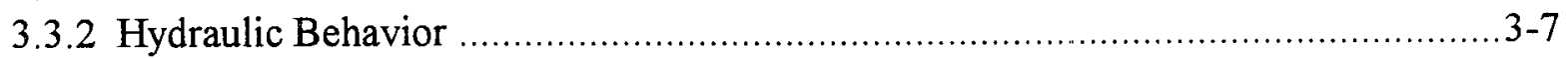

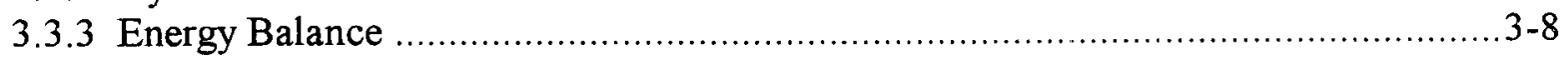

3.3.4 Assignment of Permeability Using Acoustic Emission Data ................................3-9

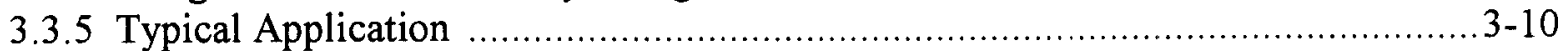

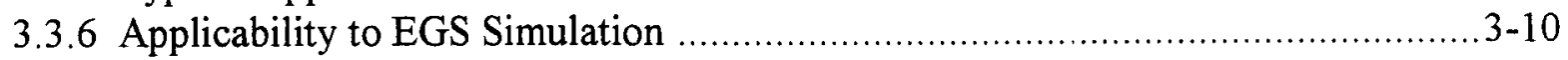

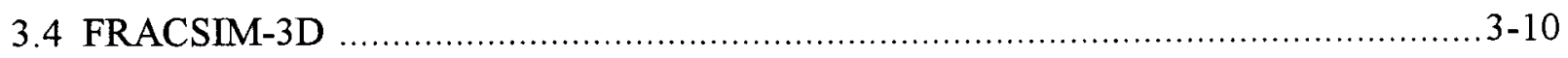

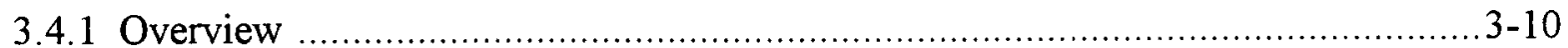

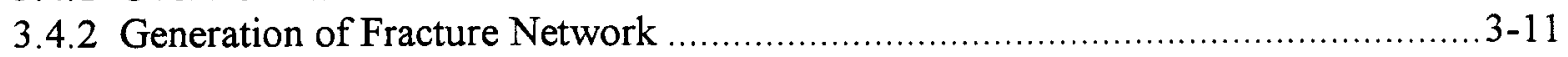

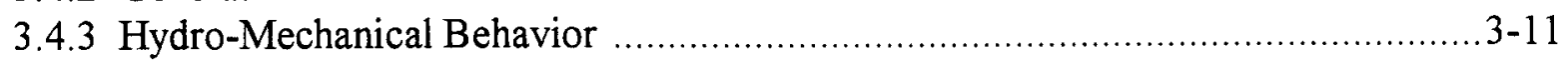

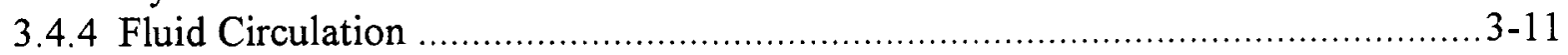

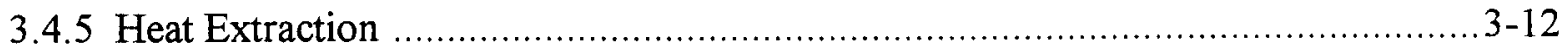

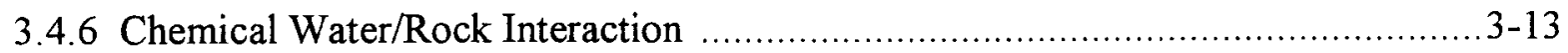

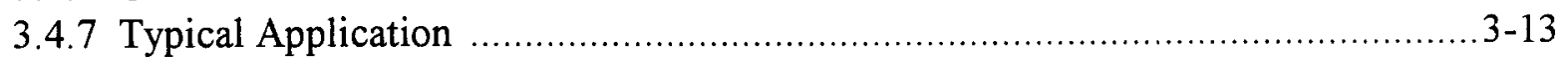

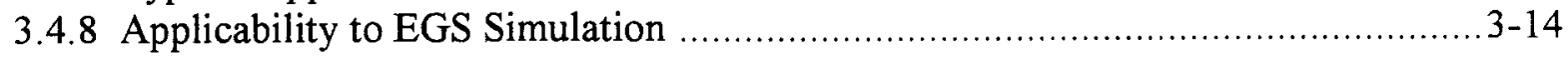

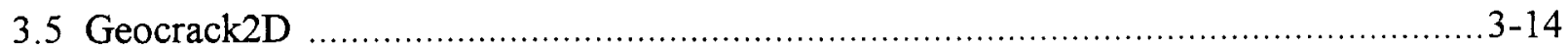

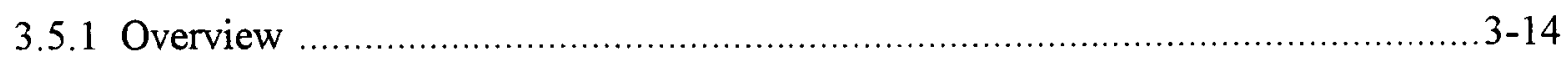

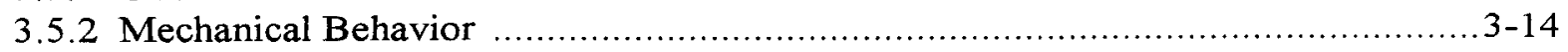

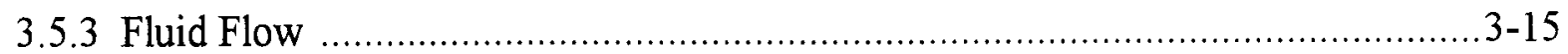

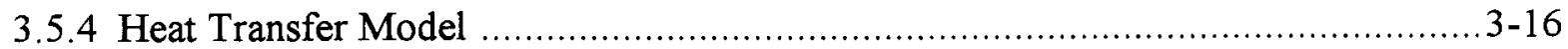

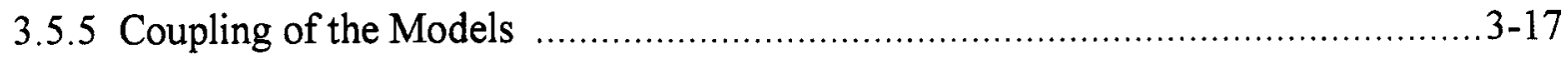

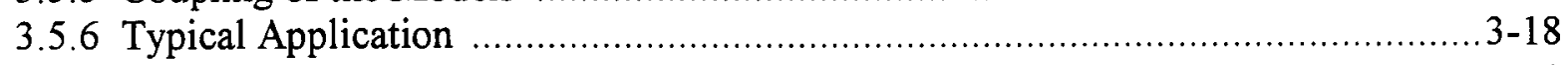

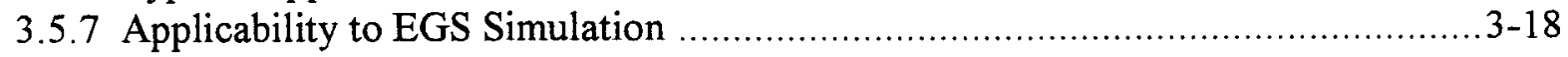

4. HYDROTHERMAL GEOTHERMAL RESERVOIR SIMULATORS ….......................4-1

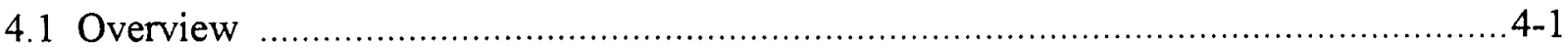

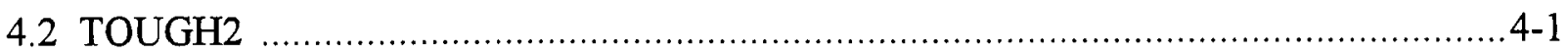

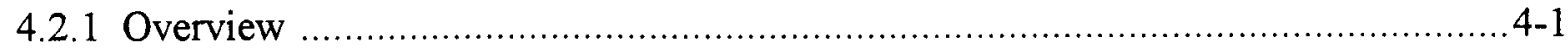

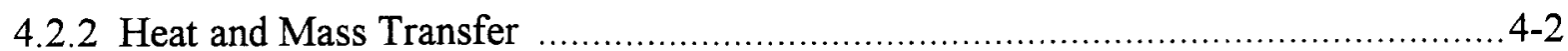

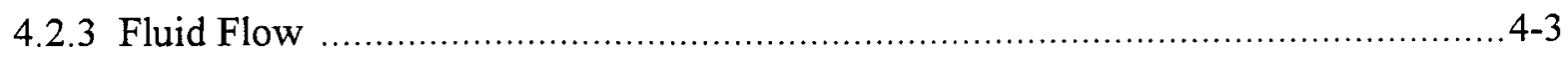


TELEPHONE: (510) 527-9876

FAX: (510) 527-8164

E-MAIL: MW@GEOTHERMEX.COM

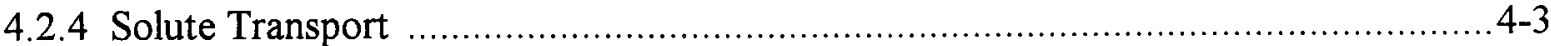

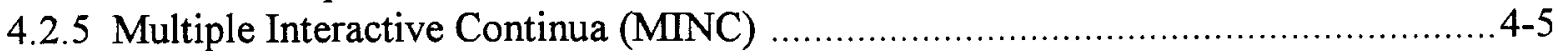

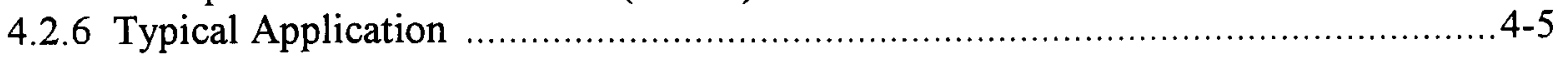

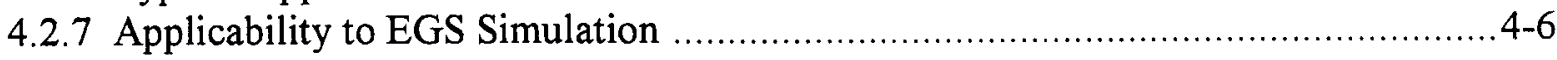

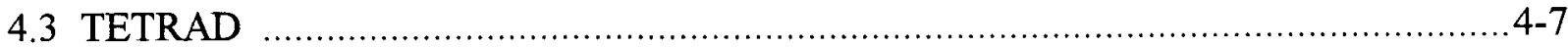

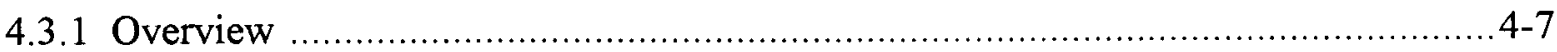

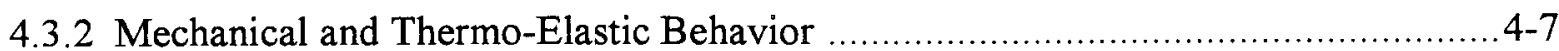

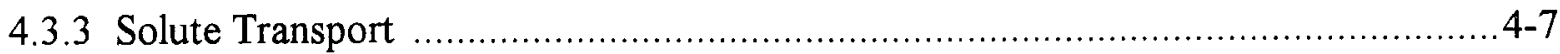

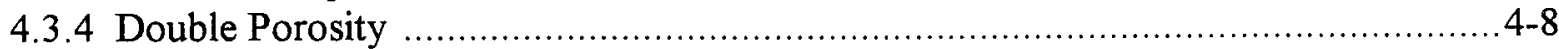

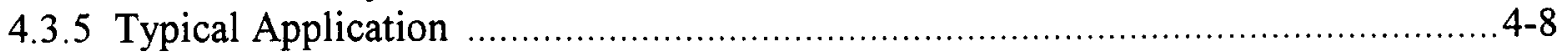

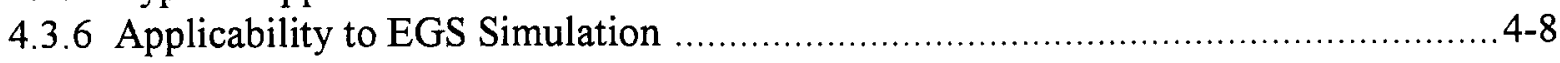

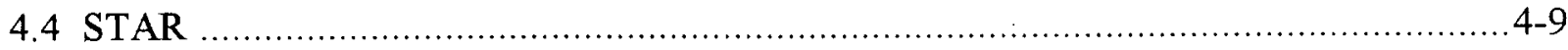

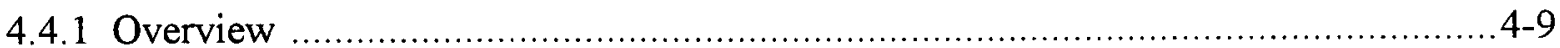

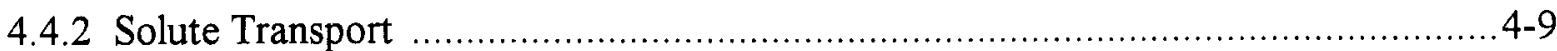

4.4.3 Permeable Matrix ........................................................................................

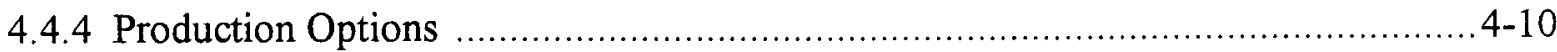

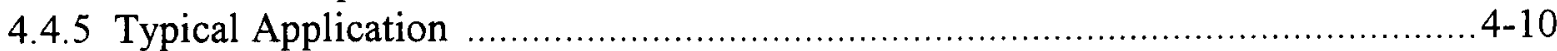

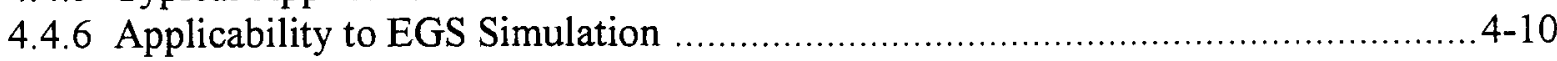

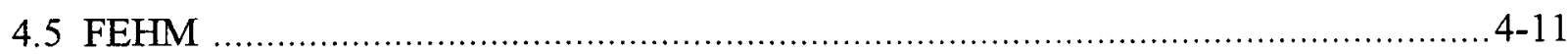

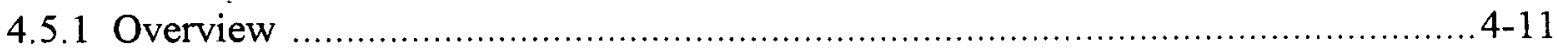

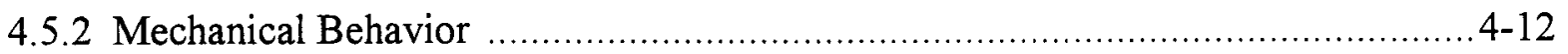

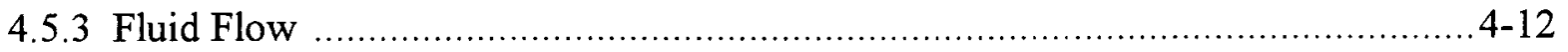

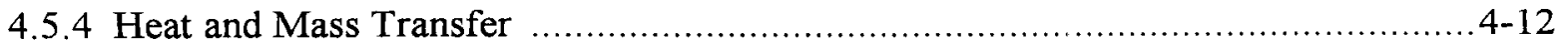

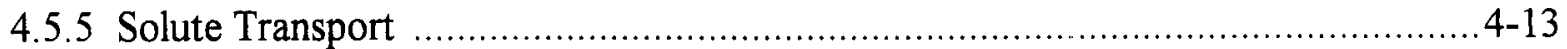

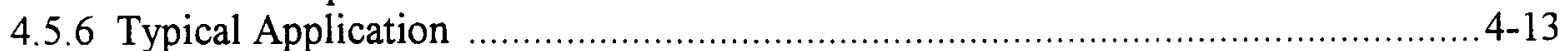

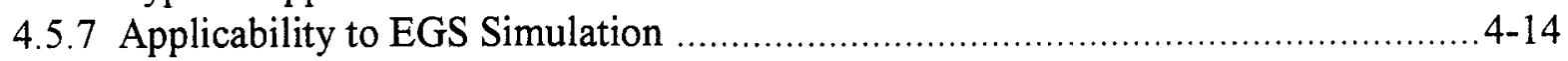

5. NUCLEAR-WASTE-ISOLATION SIMULATORS …........................................... $5-1$

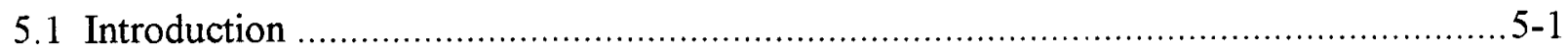

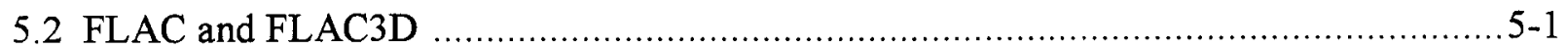

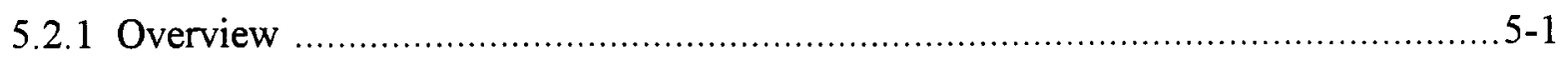

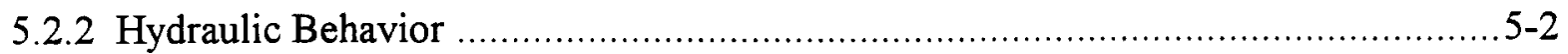

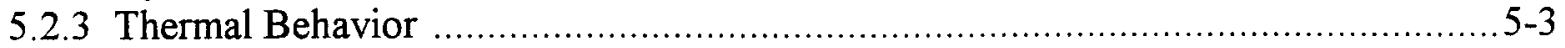

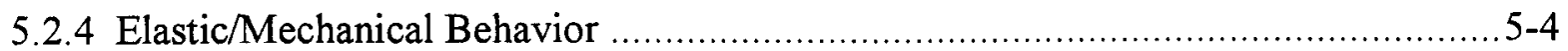

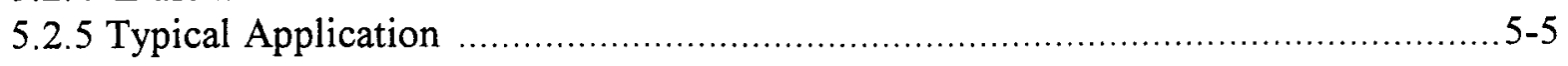


TELEPHONE: $(510)$ 527-9876

FAX: (510) 527-8164

E-MAIL: MW@GEOTHERMEX.COM

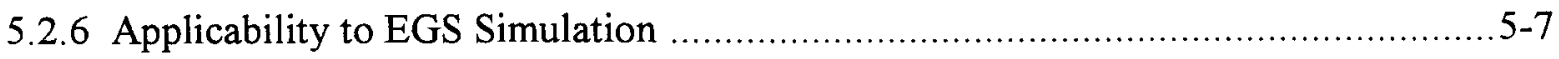

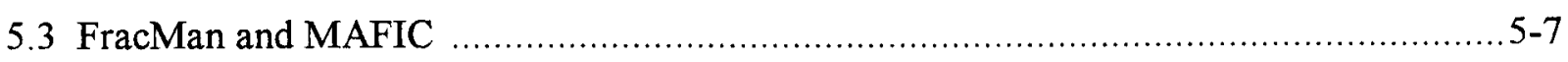

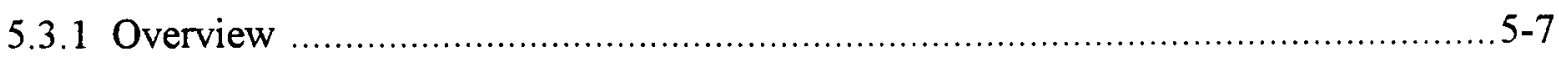

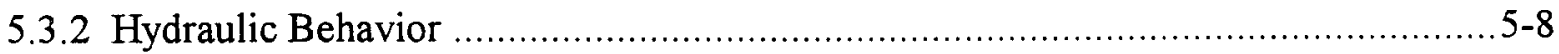

5.3.3 Elastic/Mechanical Behavior ................................................................ $5-9$

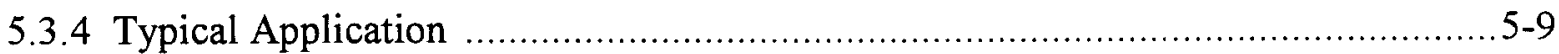

5.3.5 Applicability to EGS Simulation ...................................................... $5-10$

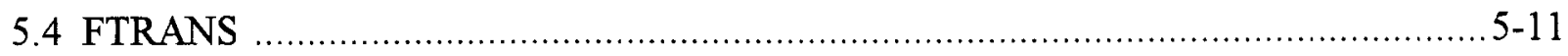

5.4 .1 Overview ............................................................................................. $5-11$

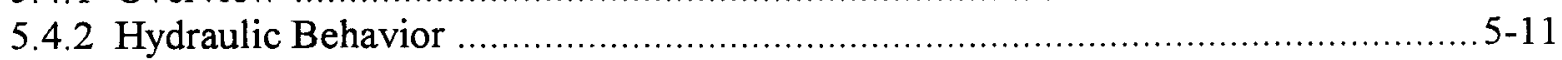

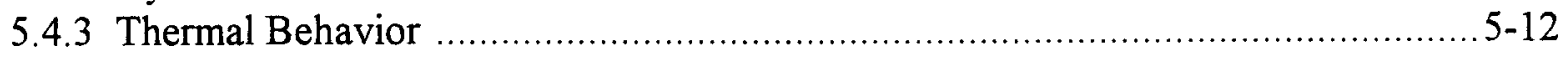

5.4.4 Elastic/Mechanical Behavior ........................................................... 5-12

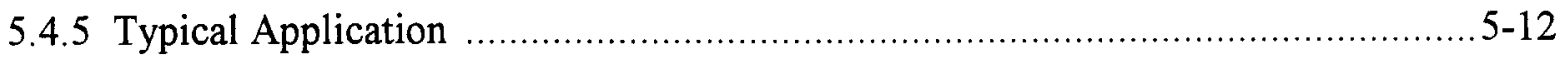

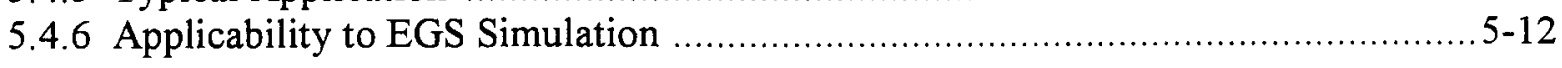

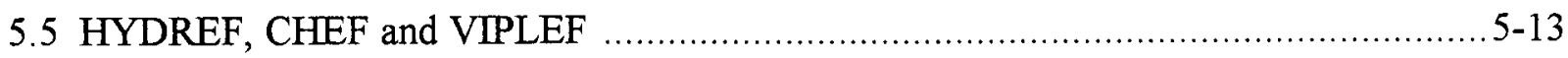

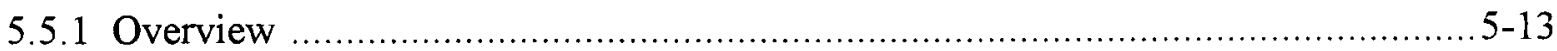

5.5.2 Hydraulic, Thermal and Elastic/Mechanical Behavior .................................. 5-14

5.5.3 Typical Application ....................................................................... 5-15

5.5.4 Applicability to EGS Simulation ...................................................... $5-16$

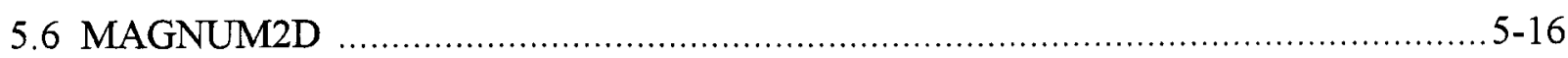

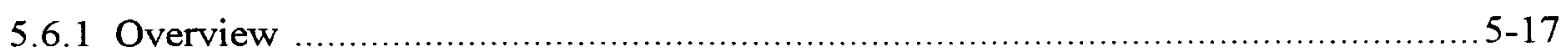

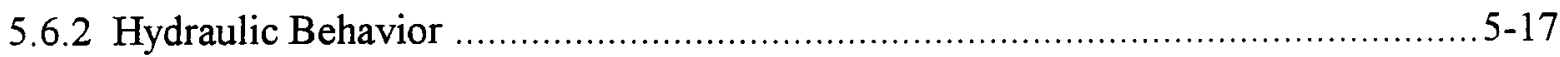

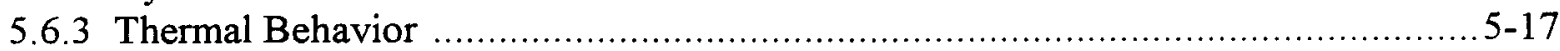

5.6.4 Elastic/Mechanical Behavior ............................................................... 5-18

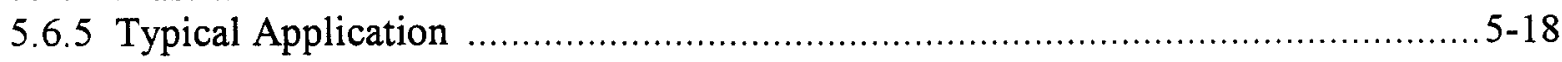

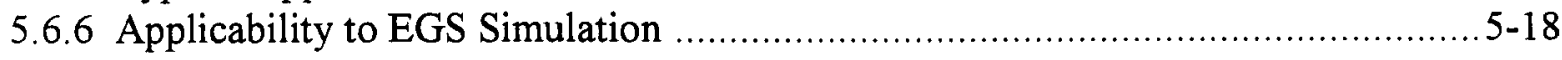

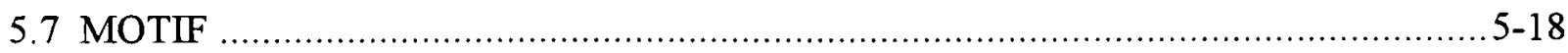

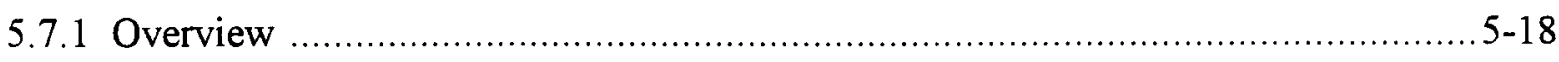

5.7.2 Hydraulic Behavior ..................................................................... $5-19$

5.7.3 Thermal/Mechanical Behavior ............................................................ 5-19

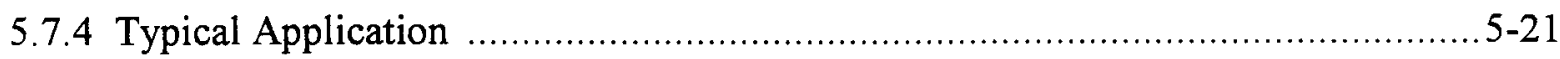

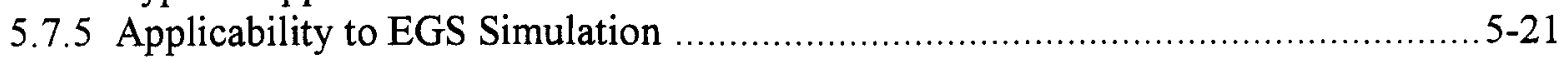

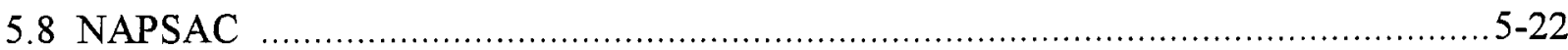




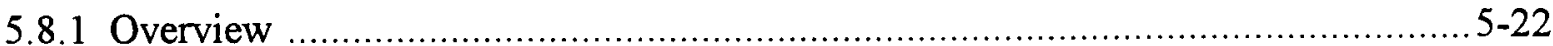

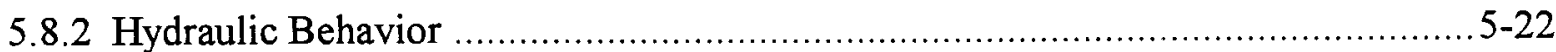

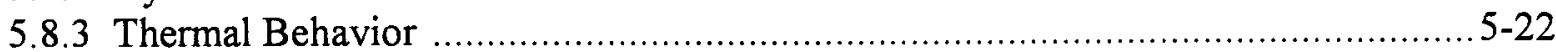

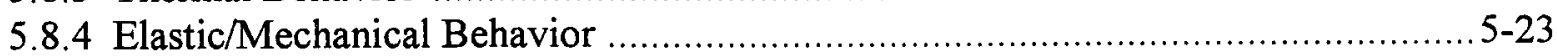

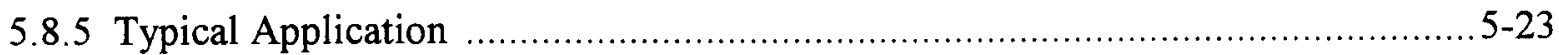

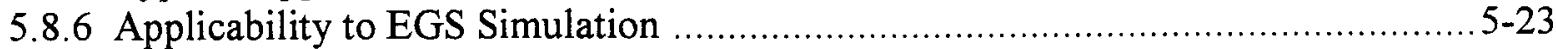

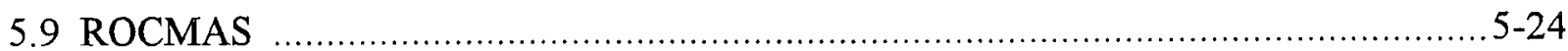

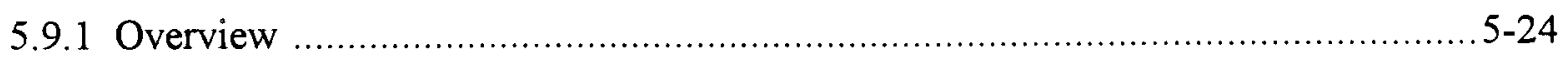

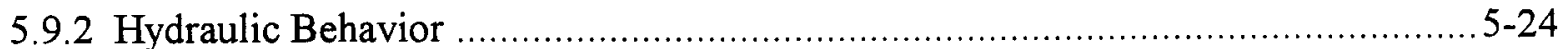

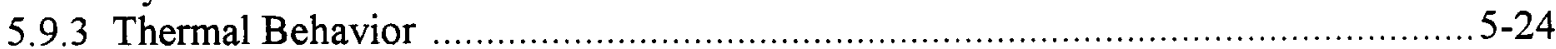

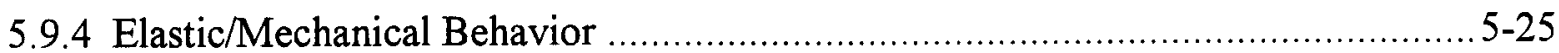

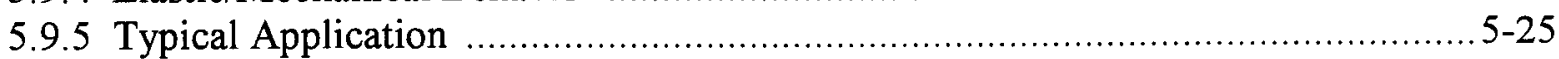

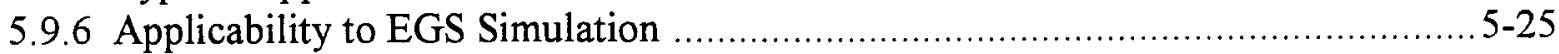

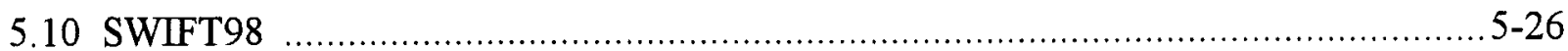

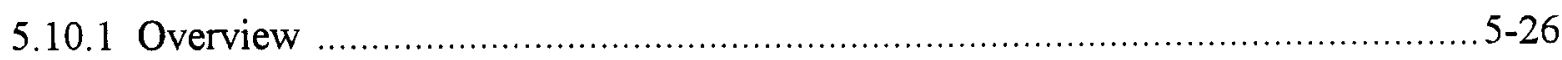

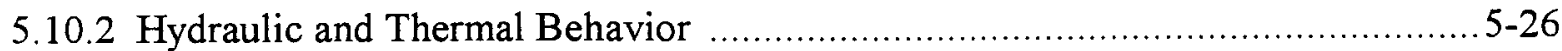

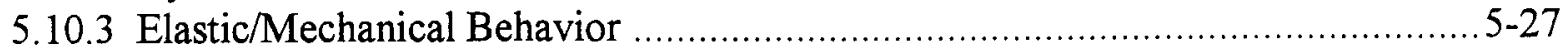

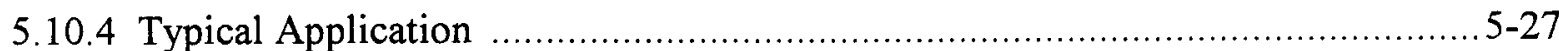

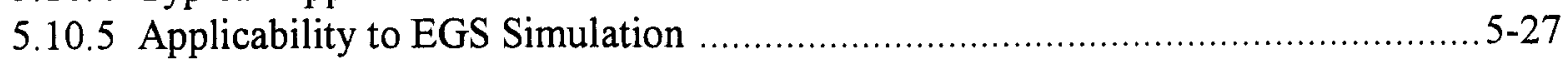

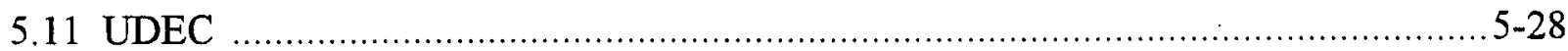

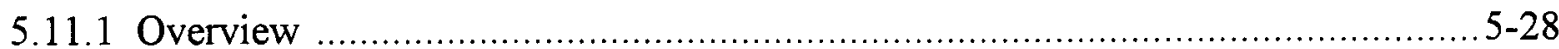

5.11.2 Hydraulic, Thermal and Elastic/Mechanical Behavior ...................................5-29

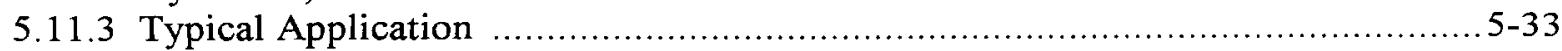

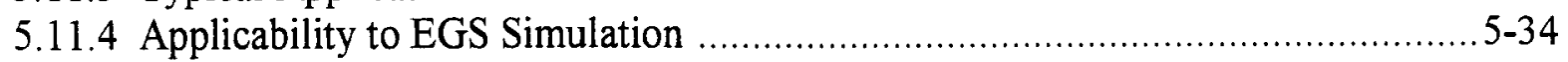

6. FEATURES OF SIMULATORS FOR ARTIFICIALLY FRACTURED SYSTEMS ..........6-1

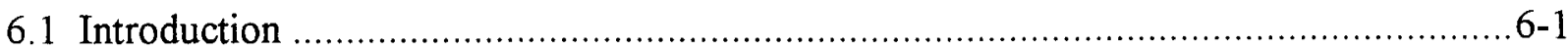

6.2 General Purpose Finite-Element Programs ............................................................ 6-1

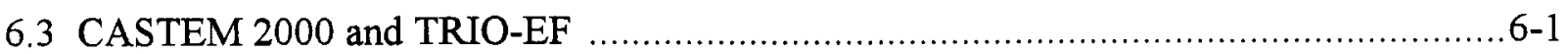

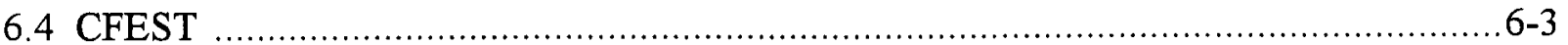

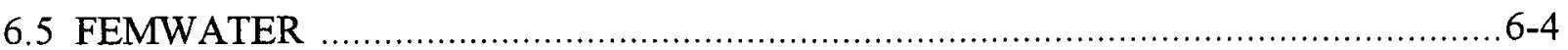

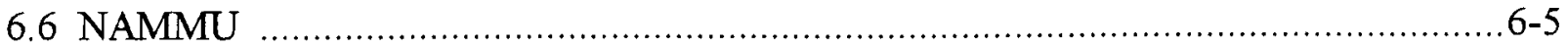

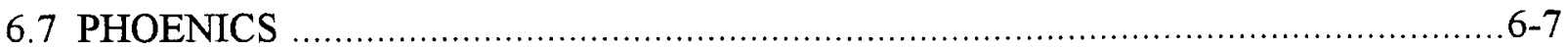


E-MAIL: MW@GEOTHERMEX.COM

6.8 PORFLOW W

6.9 SUTRA 6-11

6.10 THAMES $6-13$

6.11 TRACR3D $6-14$

7. OBSERVATIONS AND DISCUSSION $7-1$

7.1 Catergorization $7-1$

7.2 Current Capabilities Relative to Desired EGS Features ................................... 7-2

7.2.1 Explicit Representation of Fractures .................................................. $7-2$

7.2.2 Fracture Opening as a Function of Effective Stress ........................................ $7-3$

7.2.3 Shear Deformation and Associated Jacking of Fractures ............................... 7-3

7.2.4 Relationship Between Fracture Aperture and Conductivity ........................... 7-3

7.2.5 Channeling in Fractures ..................................................................... $7-3$

7.2.6 Thermo-Elastic Effects .................................................................... 7-4

7.2.7 Mineral Deposition and Dissolution ........................................................ 7-4

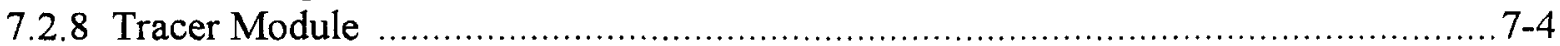

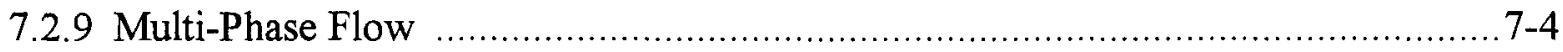

7.3 Current Capabilities Relative to Desired EGS Features ...................................... 7-4

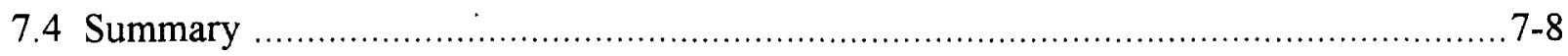

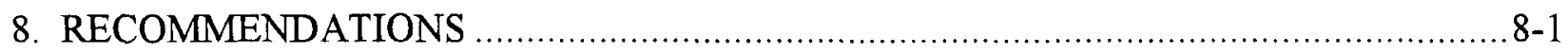

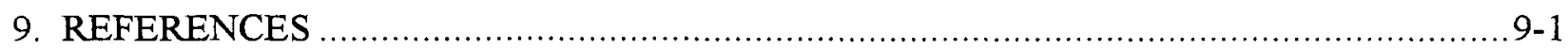

APPENDIX A: Contractual Information

APPENDIX B: Comments on the Draft Report 


\section{ILLUSTRATIONS}

Tables

1 Features of HDR Simulators Potentially Applicable to EGS

2 Features of Hydrothermal Simulators Potentially Applicable to EGS

3 Features of Nuclear-Waste-Isolation Simulators Potentially Applicable to EGS

\section{Figures}

1 Cooling on surface of fracture

2 Cooling at production well as a result of fracture surface cooling

3 Vertical section of Hijiori reservoir looking north

4 Flow rates and temperature measurement in HDR-2a (from GERD, 1997)

5 Flow rates and temperature measurement in HDR-3 (from GERD, 1997)

6 Results of the 2-D single fracture geometry run

7 Results of the 3-D multiple fracture geometry run

8 Example showing permeability distribution

9 Resulting pressure contour and flow vectors at $3430 \mathrm{~m}$ depth

10 Streamline originating from the two injection open hole intervals

11 Time histories of the computed and measured flow rates

12 Generated 3D fracture network

13 Relationship of fracture opening to shear displacement

14 Calculation of equivalent permeability 
E-MAIL: MW@GEOTHERMEX.COM

15 Comparison of measured acoustic emission pattern and predicted stimulation volume at Hijiori reservoir

16 Long-term thermal drawdown

17 Geocrack2D model of Hijiori reservoir

18 Temperatures in reservoir after 720 days of operation $\left(\min =70^{\circ} \mathrm{C}, \max =278^{\circ} \mathrm{C}\right)$

19 Calculated LTCT production temperatures from HDR-2a

20 HDR-2a 1995 PTS data showing cooling at lower fracture

21 Schematic representation of MINC partitioning using TOUGH 2

22 Three-dimensional view of the simulation model for Cerro Prieto

23 Example of modeling with an irregular grid

24 An example of grid block refinement using TOUGH

25 Grid layout for a numerical model of The Geysers geothermal field

26 Example of pressure history matching

27 Initial-state model of the Beowawe hydrothermal field, developed using TETRAD

28 Problem definition for FEHM example

29 Comparison of FEHM production well temperatures with other solutions

30 Comparison of FEHM production and observation well pressure drops with other solutions 
E-MAIL:MW@GEOTHERMEX.COM

\section{EXECUTIVE SUMMARY}

To determine if the lack of certain simulation capabilities is hindering the development of Enhanced Geothermal Systems (EGS), a review of numerical simulators used for Hot Dry Rock (HDR), hydrothermal and nuclear waste isolation applications was made. Required and desirable features for developing numerical models of EGS reservoirs were summarized, and codes in each of the three categories were reviewed to determine their capabilities. Four HDR codes (FRACTure, GEOTH3D, FRACSIM-3D and Geocrack2D), four hydrothermal codes (TOUGH2, TETRAD, STAR and FEHM) and 19 simulators used in nuclear waste isolation applications were evaluated. The need for including the desirable features was evaluated, and recommendations were made for improving the state-of-the-art relative to EGS simulation.

Enhanced Geothermal Systems is a term that has been adopted to describe geothermal systems that require enhancement to render them commercially feasible for development or continued exploitation. These differ from currently developed hydrothermal systems in that permeability may be too low for commercial exploitation, natural fluid may be absent because of a lack of fractures, or the reservoir may have become fluid-depleted as a result of over-production. However, a vast amount of heat energy remains in such systems; through artificial enhancement of the system, some of this heat can be recovered. In the near term in the United States, EGS are likely to be developed in low-permeability and/or fluid-deficient areas within or around existing hydrothermal developments. This will advance the state of EGS knowledge while providing needed improvements in energy recovery for existing generating facilities, thus achieving both the short- and long-term goals of DOE's EGS program.

It is important to establish a framework for discussion and to identify the common characteristics of EGS reservoirs that are important to successful operation. The general concept is a reservoir system consisting of a porous medium, generally with a natural fracture network, perhaps intersected by highly conductive, hydraulically induced artificial fractures. The flow occurs primarily in the fractures and is dependent on the fracture apertures, which in turn may be 
TELEPHONE: (510) 527-9876

FAX: (510) 527-8164

E-MAIL: MW@GEOTHERMEX.COM

functions of the fluid pressure and thermal contraction of adjacent rock. In EGS systems, the main challenges are improving permeability through enhancement of natural fractures or creation of artificial fractures, and optimizing heat recovery by the sweep of injection fluid through the fracture system.

Considering that EGS development in the near term will occur in or near geothermal fields that have been developed for power generation, it is likely that the simulator will have to have the basic capabilities required of a conventional hydrothermal reservoir simulator (the ability to handle multi-phase fluid flow, heat transfer and tracer transport in porous or fractured media in three dimensions). In addition, there are desirable special characteristics of an EGS reservoir simulator, including:

- explicit representation of fractures;

- the ability to change fracture opening as a function of effective stress;

- the ability to handle shear deformations and associated jacking of the fractures;

- a relationship between fracture aperture and fracture conductivity;

- channeling of flow in fractures;

- the ability to handle certain thermo-elastic effects; and

- the ability to handle mineral deposition and dissolution.

While not all are needed for a given simulation effort, a complete simulation tool would have all of these features. The inclusion (or lack thereof) of many of these features depends in some cases upon the way fractures are handled in the simulators. The codes reviewed herein use four approaches:

Discrete representation of the fractures and rock matrix, which attempts to directly model each significant fracture and to directly model the rock matrix. This approach assumes a detailed 
TELEPHONE: (510) 527-9876

FAX: (510) 527-8164

E-MAIL:MW@GEOTHERMEX.COM

knowledge of the reservoir; if that is the case, the model should provide realistic simulations with fewer approximations. The challenges are to develop methods to easily create such models and then to obtain solutions. In addition, the conceptual model (on which the numerical model is based) must be detailed enough to specify which fractures control fluid flow, and their individual characteristics.

Focus on the rock matrix ${ }_{2}$ using approximations for fracture flow. Blocks of fractured rock are modeled by specifying fracture spacing and the hydraulic properties of both matrix and fractures in a dual porosity/dual permeability approach, thus yielding effective hydraulic properties for the block. The advantage is that such an approximation allows relatively simple representation of a reservoir, providing rapid solutions in situations where the rock matrix is highly fractured or porous. Information about specific fractures is not required. However, the detail lost in making the porous flow approximation can lead to more optimistic reservoir predictions than warranted, although the MINC method of TOUGH2 circumvents some of these problems.

Using a porous-medium simulator to model large-scale, discrete fractures. Geothermal systems with such features can be represented in a numerical model using an appropriately arranged set of long and narrow gridblocks with very high porosity and permeability. This approach has proven satisfactory for several hydrothermal projects.

Focus on the fractures, using approximations for the matrix. These simulators typically use a stochastic approach to develop a fracture network model, sometimes with thousands of fractures. The fractures provide the connections in the global model, and each fracture is associated with a local rock matrix (similar to the dual-porosity approach). The advantage is that complex fracture networks can be modeled, the disadvantage is the approximation to the matrix.

Explicit Representation of Fractures. Nearly all of the reviewed simulators can be used to model fractures at some level, with the mathematical formulation that describes the fractures and the ease with which fractures can be represented differing as described above. Two of the HDR simulators (FRACTure and Geocrack2D) can represent fractures discretely; FRACSIM-3D does 
TELEPHONE: (510) 527-9876

FAX: (510) 527-8164

E-MAIL: MW@GEOTHERMEX.COM

so in simulating hydraulic fracturing operations only. Several of the nuclear-waste-isolation codes (FracMan, HYDREF, MAGNUM2D, MOTIF, PHOENICS, ROCMAS and UDEC) allow discrete fractures to be represented. Like all four hydrothermal codes, GEOTH3D uses a porous medium approach; FRACSIM-3D also uses this method to simulate normal production and injection (as opposed to stimulation).

All of the porous-medium simulators allow approximate representation of large-scale discrete fractures using long and narrow gridblocks with high porosity and permeability. Although this approach has been used to model systems in which flow is dominated by a few, major fractures, it is cumbersome to implement in systems with many fractures. At least one fracture mesh generator (Golder Associates' FracMan) has been adapted to two of the hydrothermal codes (TOUGH2 and FEHM), enabling them to represent fractures explicitly as a series of 2-D, triangular elements. This has not been used extensively as far as we know; however, this type of approach holds promise for easing the development of hydrothermal models with many discrete fractures.

Fracture Opening as a Function of Effective Stress. This feature enables a more accurate representation of reservoirs with low natural permeability or when permeability enhancements are being modeled. Three of the four HDR simulators and many of the nuclear-waste-isolation simulators include approximations of this, either through permeabilities that are a function of stress or by discrete-fracture modeling. None of the hydrothermal simulators have this feature because they do not incorporate deformation of the rock matrix, which is needed to calculate aperture changes. A significant effort would be required to implement deformation in these codes, and it may not be possible in all codes.

Shear Deformation and Associated Jacking of Fractures. Of the HDR simulators, FRACTure and FRACSIM-3D include this feature, which is particularly important as fractures grow during stimulation operations. As in the case of fracture opening in response to changes in fluid 
TELEPHONE: (510) 527-9876

FAX: (510) 527-8164

E-MAIL:MW@GEOTHERMEX.COM

pressure, none of the hydrothermal simulators can model this, nor can any of the nuclear-wasteisolation simulators.

Relationship Between Fracture Aperture and Conductivity. Three of the four HDR simulators and several of the nuclear-waste-isolation simulators use the cubic law to define the relationship between fracture aperture and conductivity; however, the cubic law cannot be used for two-phase flow. In multi-phase porous-flow models, ignoring capillary pressure, fluid flow in a fracture can be expressed a modified (multi-phase) form of Darcy's Law. However, this approach only allows the fracture characteristics to be assigned as a hydraulic property of an appropriately arranged gridblock set; it would not be a dynamic feature as in the simulators that use the cubic law. In theory, it is possible (with suitable experimentation) to develop empirical relationships for differing amounts of steam and water that would allow the cubic law to be adapted for twophase flow. However, considering the level of uncertainty that continues to be associated with the relative permeabilities of water and steam in porous media after many years of work, it is anticipated that such research would be a long-term effort.

Channeling in Fractures. Only one HDR simulator (FRACTure) handles this feature, and does so approximately by manually adjusting fracture element properties. None of the hydrothermal simulators take account of channeling. In two nuclear-waste-isolation simulators (FracMan and HYDREF), channeling is accounted for by using pipe-like elements, often located at the intersection of two fractures. There are two difficulties associates with representing channeled flow in a numerical simulation. First, one must define where channeling is occurring from field data. Although certain pressure transient analysis methods can indicate channel-like (i.e., onedimensional) flow, the location and orientation of the channel can only be inferred. Obtaining sufficient detailed knowledge to successfully identify when channeling is occurring will require input from other fracture detection and characterization technologies, which are under development but may not be useful in the near future. Second, the simulation mesh must be fine enough to capture the sharp gradients associated with flow in a small channel, and the inclusion 
TELEPHONE: (510) 527-9876

FAX: (510) 527-8164

E-MAIL:MW@GEOTHERMEX.COM

of small, cylindrical elements with random orientations presents difficulties in regard to both designing the grid and accurately computing the results.

Thermo-Elastic Effects. All of the HDR simulators except GEOTH3D include this feature; FRACSIM-3D handles thermo-elastic effects using a global stress rather than a local elasticity solution. All of the conventional hydrothermal simulators can approximate this effect by varying bulk porosity and permeability with pressure and temperature. However, they cannot simulate, for example, the thermal contraction of impermeable rock, which changes the aperture (and therefore, possibly, the conductivity) of a fracture. Many of the nuclear-waste-isolation simulators handle thermo-elastic effects.

The stress imposed by thermal effects, in addition to that imposed by fluid pressure, can alter the fracture aperture, which changes the fluid flow in the fracture. Since aperture cannot be measured directly, it must be inferred through the transient and steady-state flow simulation and by comparison with tracer data. In simulators that handle discrete fractures, the thermo-elastic effect on fracture aperture can and has been implemented. In the conventional hydrothermal simulators, bulk porosity and permeability can vary with pressure and temperature, but impermeable blocks would not be subject to any such effects. As in the case of allowing fracture apertures to change with effective stress, deformation would have to be incorporated into the hydrothermal simulators to enable them to effectively represent the thermal contraction of hot matrix blocks (and the resulting change in fracture aperture between the matrix blocks) in response to injection of cold water.

Mineral Deposition and Dissolution. Only one of the HDR simulators (FRACSIM-3D) includes a simple mineral deposition and dissolution with user-specified temperature-dependent reaction rate constants and saturation concentrations. One nuclear-waste-isolation simulator (PORFLOW W) has this capacity also. Of the hydrothermal simulators, a reactive chemical transport model has been developed to work with TOUGH2. This augmented simulator (TOUGHREACT) permits a wide range of chemical processes to be modeled, including mineral deposition and 
dissolution. After further evaluation and testing, it will be available for use with TOUGH2 and will operate in an iteratively coupled mode. Mineral precipitation and dissolution is calculated on a gridblock-by-gridblock basis; porosity and permeability are not affected. However, estimates of the effect on porosity could be made fairly easily. Implementing a full set of chemical equilibria and reaction kinetics within TOUGH2 was investigated and found to be too cumbersome for practical use.

The difficulty encountered in trying to solve chemical reactions within a numerical model of a geothermal system suggests that a de-coupled approach would be preferable if such a feature is to be implemented. However, the lack of this feature is not a hindrance to EGS development. In fact, in more than 40 years of operating hydrothermal systems, which are much more likely to have scaling problems than HDR reservoirs owing to the higher enthalpy and fluid chemistry, scaling is an operational consideration but never a serious impediment to development. The most detrimental mineral precipitation occurs upon boiling in the wellbore or reservoir. There are no hydrothermal reservoirs we know of where mineral deposition has had a significant effect on permeability, except in the region immediately adjacent to the wellbore. Even at Cerro Prieto, which is the most extreme documented case where the boiling front has clearly moved into the reservoir, scale is removed from the near-wellbore region by acid jobs and other techniques; the permeability of the reservoir has not been affected.

Tracer Module. All the reviewed simulators handle tracers fairly effectively; therefore, no further discussion is provided here.

Multi-Phase Flow. All the conventional hydrothermal simulators and a few of the nuclear-wasteisolation simulators provide multi-phase flow capability. None of the HDR simulators have this ability, because it has not been necessary in evaluating HDR reservoirs to date. However, this is likely to become a limitation if HDR simulators are to be considered for evaluating EGS projects adjacent to existing hydrothermal systems with extensive two-phase conditions. New governing 
TELEPHONE: $(510) 527-9876$

FAX: (510) 527-8164

E-MAIL:MW@GEOTHERMEX.COM

equations for two-phase flow could be incorporated into HDR simulators, but would require a significant effort.

It is apparent from the above discussion that, while each of the simulators has many of the capabilities listed above, none has all of them; each simulator has its strengths and weaknesses. Furthermore, the ease of implementing features varies with simulator type. Fortunately, a "perfect" simulator that incorporates all of the above features is not needed for most EGS projects or at every stage of a given project. For example, in the early development stage of an EGS project, when available information is limited and the primary need is for reserves estimation and project planning, porous-media or fracture-network simulators would be more practical to use. In a more mature stage of the same project, when reliable information on fractures becomes available, discrete-fracture models may become preferable for optimizing the injection/production strategy.

It is our opinion that at this time, further development of existing simulators is more useful than developing a single, all-purpose simulator for EGS applications. This is particularly true considering that near-term EGS development in the US is likely to take place in hot, lowpermeability areas in or around existing hydrothermal fields. Here, a field operator will need to use numerical simulation to predict the effect of the EGS development on conditions in the main part of the reservoir. Considering that nearly all reservoirs developed for geothermal power production have two-phase conditions, a conventional hydrothermal simulator must be used for the present. Such simulators have the proven capability and robustness to handle perhaps thousands of gridblocks and hundreds of wells, and there is already a level of familiarity with their use by the field operators. Although such simulators will have to handle the fracture-related features in an approximate way and cannot represent the dynamic aspects of fractures, this should not hinder the advancement of EGS developments collocated with hydrothermal projects.

In the longer term, a stand-alone EGS project might require a dedicated EGS simulator that combines the capabilities of HDR and hydrothermal simulators, and possibly some of the 
features of the more complex nuclear-waste-isolation simulators. If hydraulically active fractures could be identified and characterized, then it would be appropriate to pursue including some of the fracture-related features (fracture opening as a function of effective stress, the relationship between fracture aperture and conductivity, shear deformation and channeling). As research into the identification and characterization of hydraulically active fractures continues, these simulator features will become more important than they are now, because there will be a practical basis for their application.

No EGS reservoir has operated for sufficient time to validate any numerical model, fracturebased or otherwise. Therefore, at the present time, whether or not a particular simulator has the ability to model discrete fractures or to vary the characteristics of those fractures is not hindering the development of EGS. Developing an EGS simulation experience base should be the highest priority at this time. We strongly believe that meaningful reservoir modeling and simulator development cannot done in the abstract. Meaningful modeling is only done as an active participant in the development and operation of a reservoir. Only through interaction with realistic problems can the appropriate simulation needs be identified and the skills developed to apply them to other reservoirs.

Therefore, our primary recommendation is that DOE support active simulation of real EGS reservoirs. This could be done either as part of ongoing international projects, such as those in Japan and Europe, or as part of future EGS development in the US or elsewhere. The active participation of the field developer/operator would be required. We envisage a situation where, for a specific, active project, experts familiar with each of the categories of simulators (porousmedium, discrete-fracture, and fracture-network) would develop models of an EGS, applying their technologies to simulation of reservoir operation and prediction of future reservoir behavior. The teams would meet regularly to exchange data and concepts. This "hands-on" experience will provide the background necessary to demonstrate what simulators are appropriate at different stages of reservoir development and what features need to be added to 
TELEPHONE: (510) 527-9876

FAX: (510) 527-8164

E-MAIL: MW@GEOTHERMEX.COM

improve the accuracy of the simulation effort. Funding could then be provided to develop these additional features.

At the end of such a project, the field operator(s) would have knowledge of the capabilities of the different simulators. The simulators that perform the best would be favored for similar projects in the future; thus, a combination of real-world experience and market forces would decide which approaches are most valuable.

The mathematical basis of fracture-network and discrete-fracture simulators allows the detailed specification of the fracture geometry, which is useful and appropriate when detailed knowledge of in-situ fractures is available. Therefore, we also recommend that further research should be funded for improving both fracture-network simulators and discrete-fracture simulators for EGS use. Potential areas of improvement include the ability to: 1) handle two-phase flow (including experimentation to adapt the cubic law for two-phase conditions); 2) simulate the formation of a hydraulically stimulated fracture network, given appropriate stress information; and 3) modify fracture aperture as a function of both effective and shear stress.

For hydrothermal simulators, the ability to handle rock deformation could be added, which would enable them to be used to model the effect on fracture apertures of : 1) thermal contraction of matrix blocks; 2) changes in effective stress; and 3) changes in shear stress. Furthermore, the use of hydrothermal codes to represent discrete, hydraulically active fractures could be investigated further, perhaps by adapting a fracture network generator to the porousmedium solver. The link already developed between FracMan (fracture network mesh generator) and TOUGH2 or FEHM (solvers) could be investigated to determine its utility in EGS evaluations. 
TELEPHONE: (510) 527-9876

FAX: (510) 527-8164

E-MAIL:MW@GEOTHERMEX.COM

\section{INTRODUCTION}

\subsection{Background}

This report reviews existing reservoir geothermal simulation technology applicable to the analysis of Enhanced Geothermal Systems (EGS), a term that has been adopted to describe geothermal systems which require enhancement to render them commercially feasible for development or continued exploitation. These differ from currently developed hydrothermal systems in that permeability may be too low for commercial exploitation, natural fluid may be absent because of a lack of fractures, or the reservoir may have become fluid-depleted as a result of over-production. However, a vast amount of heat energy remains in such systems; through artificial enhancement of the system, some of this heat can be recovered.

The goal of the proposed work is to recommend research needed either to: 1) develop a new numerical simulator; or 2) modify one or more of the existing reservoir simulators, so that enhanced geothermal systems can be modeled accurately. Details of the scope of work are provided in Section 1.2. Existing hydrothermal simulators cannot accurately model dynamic fracture characteristics; existing Hot Dry Rock (HDR) simulators are better at handling fracture systems, but lack certain other critical features.

Until a sufficient level of simulation accuracy is achieved, commercial development of many enhanced geothermal systems, particularly those at the low-permeability end of the EGS spectrum, will not be realized. Commercial geothermal companies are unwilling to invest in such EGS resources because there is no reliable way to predict their future performance. Accurate prediction is hindered by inadequate simulation technology, and commercial entities are unwilling to develop specialized software for EGS without the justification of a reasonable payoff, which can only be estimated by accurate prediction of resource behavior. DOE can break this cycle by undertaking needed research in the field of numerical simulation. This will enable a reliable, early evaluation of EGS projects, thus stimulating interest from development 
TELEPHONE: (510) 527-9876

FAX: (510) 527-8164

E-MAIL: MWQGEOTHERMEX.COM

companies and setting the stage for their commercialization. However, it must also be recognized that each reservoir is unique and therefore, each simulation is an iterative process that develops as more is learned about a reservoir. Simulation and operational experience must be used together to obtain reliable models of any particular reservoir.

For conventional geothermal resources (hot water, steam and two-phase reservoirs in porous or fractured rock), reservoir simulation is a routine activity. This report reviews the applicability of such conventional simulators to EGS developments. For fluid-deficient EGS developments, conventional simulators have been used to design and manage injection of water from outside sources. Conventional simulators have also been used to model complex fracture systems with certain idealization; that is, in an approximate way.

However, conventional simulators have not been used extensively for modeling artificially fractured systems, and/or those whose hydraulic characteristics vary with different operating conditions. Much can be learned about modeling EGS reservoirs from the experience gained in modeling of artificially fractured systems in connection with HDR projects, which lie at one extreme of the EGS spectrum. Some of the simulation methods developed for HDR systems have application in the broader area of EGS reservoirs. Numerical simulation is also a routine activity in nuclear waste isolation projects. Models of EGS and nuclear waste isolation systems have much in common; for example, the latter also deals with low permeability and fluiddeficient systems. Therefore, this report includes a review of simulators developed for nuclear waste isolation problems.

\subsection{Scope of Work}

The work for this study has consisted of six tasks:

Task 1: Conduct a literature search to identify the existing reservoir simulation technology for hydrothermal and HDR systems, and interview experts who are now (or have been) involved in numerical modeling of both hydrothermal and HDR systems in the U.S. and abroad. 
TELEPHONE: (510) 527-9876

FAX: (510) 527-8164

E-MAIL: MW @GEOTHERMEX.COM

Task 2: Determine the utility and limitations of each of the hydrothermal and HDR simulators developed to date, particularly as regards their handling of fracture definition and fluid flow and heat transfer in artificial fractures.

Task 3: Prepare a report documenting the results of Tasks 1 and 2.

Task 4: Determine if there are simulators developed anywhere in the world (either publicly available or proprietary) for contaminant transport or nuclear-waste isolation that would have application to simulation of enhanced geothermal systems. Possible areas of application include accurate geometric representation of fracture networks, geometric representation of in-plane fracture heterogeneity, and analogs for heat transfer in multi-rate matrix diffusion.

Task 5: Recommend specific enhancements to existing simulators and/or development of new simulators which would enable better modeling of the entire spectrum of enhanced geothermal systems.

Task 6: Prepare a complete report documenting the results of Tasks 1 through 5.

\subsection{Acquisition of Information}

The authors have varying levels of familiarity with the codes reviewed. GeothermEx has performed numerous simulations of geothermal reservoirs using both TETRAD and TOUGH2 (and its predecessors), and therefore used a combination of practical experience and information in the user's manuals for this review. Daniel Swenson and Brian Hardeman of Thunderhead Engineering Consultants are authors of Geocrack 2D, one of the HDR codes reviewed. Golder Associates developed the FracMan and MAFIC codes, and used their extensive experience with many of the nuclear-waste-isolation simulators in preparing this report. For the other simulators, we contacted the authors directly; some authors provided extensive information, while others did not. We supplemented these sources with published information from journals and websites. 
Because of limits in budget and scope, we could not purchase the user's manual for each simulator. Therefore, for the less familiar simulators, we relied upon what was provided by the authors of the codes or obtained from public sources. The result of this is that more details are given for some codes and less for others. This is not meant to imply any bias towards or against any particular simulator, but reflects the information available to us combined with the knowledge and experience of the authors.

\subsection{Structure of the Report}

The reservoir features of importance in the operation of enhanced geothermal systems are described first (Section 2). We then review existing reservoir simulators developed for application to HDR reservoirs (Section 3), hydrothermal systems (Section 4), and nuclear waste isolation (Section 5), highlighting capabilities relevant to the evaluation and assessment of EGS. We focus on simulators that include some representation of flow in fractures, only mentioning other simulators, such as general-purpose programs or groundwater models (Section 6). Following these detailed descriptions, we summarize and comment on the simulators (Section 7), and recommend a course of action for further development (Section 8). References are included in Section 9.

Appendix A contains contractual information, including a description of the original and revised scope of work for this study. Appendix B presents comments on the draft report from DOE reviewer(s) and the replies of the authors to those comments.

\subsection{Acknowledgements}

We thank the authors of each of the codes for sharing details of their simulators with us and for providing some of the illustrations included herein. In particular, we thank Thomas Kohl (FRACTure), Takeshi Yamamoto (GEOTH3D), Z. Jing and T. Hashida (FRACSIM-3D), George Zyvoloski (FEHM), Karsten Pruess (TOUGH2) and John Pritchett and Sabodh Garg (STAR). 
GeothermEX, InC. $\begin{aligned} & 5221 \text { CENTPAL AVENUE, SUITE } 201 \\ & \text { RICHMOND, CALFORNAA } 98004-5829\end{aligned}$

TELEPHONE: (510) 527-9876

FAX: (510) 527-8164

E-MAIL: MW@GEOTHERMEX.COM

The data on Hijiori was supplied by the New Energy and Industrial Technology Development Organization, Japan. 


\section{FEATURES OF SIMULATORS FOR ARTIFICIALLY FRACTURED SYSTEMS}

\subsection{Introduction}

Before we review the simulation tools available, it is important to establish a framework for discussion and to identify the common characteristics of EGS reservoirs that are important to successful operation. The general concept is a reservoir system consisting of a porous medium, generally with a natural fracture network, perhaps intersected by highly conductive, hydraulically induced artificial fractures. The flow occurs primarily in the fractures and is dependent on the fracture apertures, which in turn may be functions of the fluid pressure and thermal contraction of adjacent rock. In EGS systems, the main challenges are improving permeability through enhancement of natural fractures or creation of artificial fractures, and optimizing heat recovery through injection. Heat is removed by the sweep of injection fluid through the fracture system.

Because flow in fractures is central to the discussion of EGS reservoirs, it is useful to illustrate this effect. Figure 1 shows a single 100-meter-long fracture with cold-water injection on the left and production on the right. Instead of uniform cooling of the rock, the surface of the fracture cools rapidly, leading to significant cooling of the produced water (figure 2). The strong temperature gradients that develop in the rock (figure 1) must be represented accurately in the model in order to correctly forecast the production temperature. The analytic solution for this heat-transfer problem is given in Carslaw and Jaeger (1959).

The importance of flow in fractures can be further illustrated using data from the Hijiori HDR reservoir in Japan (GERD, 1996; GERD 1997). The wells intersect fractures that are part of the ring structure around the southern boundary of the Hijiori caldera. The fractures strike approximately east-west and dip steeply to the north, at an angle of about 70 degrees from the horizontal. The intersections of these fractures with the wells are shown in Figure 3. HDR-2a 
TELEPHONE: (510) 527-9876

FAX: (510) 527-8164

E-MAIL: MW@GEOTHERMEX.COM

and HDR-3 are production wells that are open (not cased) below about 1,500 meters $(1,500 \mathrm{~m})$. HDR-1 is the injection well and is cased to a depth of about $2,150 \mathrm{~m}$.

Pressure-temperature-spinner (PTS) data show the distribution of flows in the fractures, as illustrated in Figures 4 and 5 (GERD, 1997). What can clearly be seen are the distinct entry points into the wells at a limited number of fractures. It is believed that fractures F2a-9/F3-7 intersect a common fracture and similarly for F2a-3/F3-2 (the steeply dipping fractures result in different depths of intersection with the two wells). During testing, it can be seen that there is significant cooling in HDR-2a as a result of flow from the injection well flowing through the F2a-9/F3-7 fracture. The important points are the clear identification of flow in fractures and the rapid cooling that can result. This is what must be captured in EGS reservoir simulators.

Pruess (1990) discusses when fractures must be represented explicitly and when they can be modeled as an effective continuum. An effective continuum approach is justified when matrix and fractures remain in approximate thermodynamic equilibrium; that is, when there are relatively low temperature gradients in the rock. For a typical situation in which the rock matrix is relatively impermeable, approximate equilibrium is only valid if the active fracture spacing is small (or flow rates are very small), owing to the relatively low conductivity of the rock. If thermal equilibration is to occur within a few months, fracture spacing must be less than 2-3 meters. In artificially fractured systems and in many low-permeability systems, the actively flowing fractures are more widely spaced (perhaps ten to a few hundreds of meters apart), so that explicit modeling of fractures is more appropriate. This can be accomplished either by a discrete-fracture formulation or through sufficient grid refinement and application of appropriate permeabilities in a porous-medium model.

Detailed discussions of EGS modeling needs were held during the Structured Academic Review of HDR/HWR (Hot Wet Rock), convened at Tohoku University in 1997 (Structured Academic Review of HDR/HWR, 1997). The approximately 70 participants in this review, largely from the academic and research communities, have combined experience with all of the HDR/HWR 
TELEPHONE: $(510)$ 527-9876

FAX: $(510)$ 527-8164

E-MAIL: MW@GEOTHERMEX.COM

reservoirs (Rosemanowes, UK; Soultz-sous-Forêts, France; Hijiori and Ogachi, Japan; and Fenton Hill, USA). Their experience and conclusions represent a significant cumulative knowledge relevant to EGS reservoirs. The relevant conclusions from this review are quoted below, followed by our interpretation of the implications for a reservoir simulator (indicated in italics).

\subsection{General Features of Interest}

- "A typical HDR site does not exist. The creation of the reservoir and its behavior during circulation is governed by the characteristics of the natural fracture system and the in-situ stresses (Willis-Richards et al., 1995). The reservoir engineering procedures are therefore likely to be site-specific."

The implication is that simulation will necessarily be an important part of reservoir design. The simulation will be iterative, with the model changing as more information is obtained about each site. Different modeling approaches will be appropriate for different reservoirs.

\subsection{Flow Paths in the Reservoir}

- "High fracture densities can be expected in most deep basement rock masses. However, experience has shown that only a small but finite fraction of the natural fractures intersected by boreholes accept flow prior to stimulation ... Thus the existence of a network of connected, open flow paths defined within the natural fracture lattice appears to be common within deep basement rocks. This is consistent with mounting evidence of significant basement permeability from many other disciplines (see special volume edited by Torgersen, 1991)."

- "The original concept of engineering HDR systems by driving parallel hydrofractures between judiciously located boreholes has not proven to be practicable. The interaction between the propagating hydrofracture and the natural fracture system appears to inhibit the 
TELEPHONE: (510) 527-9876

FAX: (510) 527-8164

E-MAIL:MW@GEOTHERMEX.COM

distance out to which hydraulic fractures can be propagated in crystalline rock. However, hydrofracture methods (including proppant injection) serve as a potential means of improving the linkage between the borehole to the natural fracture system."

- "Improvements in the hydraulic linkage between boreholes can only be engineered by opening or improving flow paths within the natural fracture system."

The model must include explicit representation of the fractures, since fractures are the primary flow paths in the reservoir. Not all fractures need to be modeled, just the ones participating in flow. In addition, the model needs to allow arbitrary orientation of the fractures.

\subsection{Change in Fracture Aperture Due to Effective Stress and Shear}

- "Fracture-normal dilation in response to reduced effective normal stress appears to have an important influence on the penetration of pressure disturbance into the rock mass during stimulation injections, and is probably the controlling influence in the vicinity of the well (about 200-300 $\mathrm{m}$ at Soultz). Thus it is an important factor influencing the geometry of the stimulated rock volume. At greater distances where the pressure change is small in comparison, shearing may dominate (Cornet and Jones, 1994; Jones et al., 1995)."

The model should include fracture opening as a function of effective stress. As the pressure in the fractures is increased, the fractures open, changing the flow in the reservoir.

- "Shear displacement occurring on natural fractures is the most credible mechanism that can account for permanent increases in the transmissivity of flow paths and, by implication, increases in the intrinsic permeability of the rock mass."

- "Shearing can occur for arbitrarily small pressure increases when fractures are present that are verging on shear failure under the prevailing stress state. Thus, shearing can be induced at comparatively large distances from the injection point, where pressure disturbance is 
TELEPHONE: (510) 527-9876

FAX: (510) 527-8164

E-MAIL: MW@GEOTHERMEX.COM

small, provided that a near-critical stress prevails and suitably-oriented fractures exist (Pine and Batchelor, 1984). The direction of shear growth can be downward or upward (or horizontal) depending on the in-situ stress gradients."

The model should include shear deformations and associated jacking of the fractures. The shear can be induced as a result of increased fluid pressure in the fractures, which reduces the normal stress.

\subsection{Flow Regime in the Fractures}

- "The relationship between fracture dilation and the resulting change in hydraulic conductivity is of fundamental importance for understanding the stimulation process in HDR reservoirs. At the time of the last review it was common practice to assume that the hydraulic conductivity of a fracture was adequately described by the cubic law for flow between smooth parallel plates with a separation the same as the mean aperture of the fracture (Witherspoon et al., 1980). This is now believed to be false. Defining the hydraulic aperture, $a_{h}$, as the equivalent parallel plate aperture that gives the same flow under the same head gradient, the 'cubic' law predicts that $a_{h}$ and mean aperture, $\langle a\rangle$, have the same value. .... The balance of current evidence suggests that hydraulic apertures are generally only a fraction of mean apertures for natural fractures (e.g., Durham and Bonner, 1994; Hakami, 1996)."

- "However, Schrauf and Evans (1986) made the important observation that although the full form of the cubic law may not hold, it is often the case that increments of closure result in changes in $a_{h}$ which are only slightly smaller, at least provided that the closure is not great. Reviews of published experimental data on flow through fractures subject to normal loading suggest that the incremental form of the cubic law may be approximately valid for hydraulic apertures greater than $50 \mu \mathrm{m}$ (Boitnott, 1991; Evans et al., 1992). At smaller hydraulic apertures, changes in $\mathrm{a}_{\mathrm{h}}$ become an increasingly smaller fraction of the mean aperture 
TELEPHONE: $(510) 527-9876$

FAX: $(510) 527-8164$

E-MAIL:MW@GEOTHERMEX.COM

change. It is perhaps time to review the available data base to attempt to improve the constraints on the validity of the incremental form of the cubic law for both normal and shear loading."

The model must include a relationship between fracture aperture and fracture conductivity. While the cubic law is a first approximation, this relationship may need to be more complex, including the potential for turbulent flow in the fractures.

\subsection{Channeling of Flow}

- "The nature of the flow field within the reservoir, and most importantly, within the planes of the fractures that link to form through-going conduits, represents one of the most crucial yet least understood aspects affecting the performance and longevity of HDR Systems. Data from rock mechanics experiments and theoretical analyses provide firm grounds to expect that channeling of flow within fractures will occur to some extent, and that this will reduce the surface area swept by the primary flow .... The available data from tracers and thermal drawdown are insufficient to define the extent to which the channeling phenomenon is a problem."

- "Channeling must take place. This is clear from the paper of Brown (1987), Matsuki et al. (1996) and Glover et al. (1996). Channeling is of course dependent on deformation (changes of aperture, shear deformation along the fractures and thermal contraction). From the viewpoint of overall flow impedance of fluid circulation stage, channeling reduces the overall flow impedance. But, the story is completely opposite from the viewpoint of heat transfer. During the heat extraction stage, heat extraction area will be limited due to channeling and thus channeling reduces the amount of heat extracted."

The flow in a fracture is obviously not uniform. To the extent that flow "channels," less surface area is accessed for heat removal, and the potential for thermal breakthrough is enhanced. The simulator needs to accommodate channeling. 
- "Cooling of the reservoir during operation will result in the generation of thermo-elastic stress. Both positive and negative consequences can be anticipated. The positive aspects include:

- A tendency to reduce the impedance of flow paths due to reduced effective normal stress.

- The promotion of thermal cracking which might serve to increase the heat transfer area (Cleary and Barr, 1982). In their laboratory investigation of thermal cracking, Zhao and Brown (1992) found the induced cracks did not penetrate sufficiently to have any significant positive influence on the long-term performance of the reservoir. However, Kohl et al. (1992) showed in their coupled numerical modeling study that substantial, penetrative reductions in compressive stress in the plane of fractures can be expected to occur during prolonged circulation as a large volume of rock cools, although the reduction was markedly less than predicted by models which neglected the elasticity of the surrounding medium. Whether thermal cracking results in the growth of significant secondary fractures depends primarily upon the initial in-situ stress conditions.

The negative aspects relate to the danger of promoting focusing of flow within the cooled flow paths. Again, more data is needed from prolonged circulation to evaluate these factors."

The simulator needs to include thermo-elastic effects, both because thermal contraction of the rock can open the fractures and change flow, and also because thermal contraction could induce thermal fractures.

\subsection{Water/Rock Chemical Interaction}

- "The chemical aspects of most HDR models remain undeveloped due to the lack of adequate data for reaction rate, the uncertainty as to the importance of ion exchange reactions and 
known inadequacies in describing the microgeometry of the chemical precipitation (e.g., clay mineral formation) and dissolution. This has limited the willingness of researchers to incorporate such chemical coupling into more comprehensive models."

- "The most important long-term factors influencing reservoir circulation could be chemical. The thickness of precipitates building up on fracture surfaces, and conversely, the depths to which those surfaces could be eroded by chemical dissolution, could become of similar dimensions to fracture apertures .... Chemical precipitation, or dissolution causing repositioning of loose debris, may significantly influence fluid flow in a reservoir in the future."

- "It may be difficult to model rates of chemical processes, but it is important to model the processes by which chemical interference may take place, and the hydraulic consequences which would be observed. If a remedy was found for such difficulties, it could be applied when they were observed, rather than waiting until the damage was done."

Rock/water chemical interactions may be significant and should be included in a complete simulation tool.

\subsection{Tracer Testing}

- "Chemical tracers offer one of the few diagnostic tools available for assessing the changes in flow patterns within a producing HDR/HWR reservoir. DuTeaux and Callahan (1996) have shown, by comparing two tracers that were injected simultaneously at Fenton Hill ( $\mathrm{p}$-toluene sulfonic acid, a conservative tracer, and sodium fluorescein, a thermally degrading compound at elevated temperatures) that changes in reservoir temperature may be identified and characterized before changes in production temperature are observed. In addition, although a field test has not yet been attempted, the comparison of an adsorbing tracer with a conservative tracer could be employed to measure changes in the surface area of the active 


\section{GeOthermEX, InC. $\begin{aligned} & \text { 5221 CENTALA AVENUE, SUITE } 201 \\ & \text { RICHMOND, CALIFORNIA } 940045829\end{aligned}$}

TELEPHONE: (510) 527-9876

FAX: (510) 527-8164

E-MAIL: MW GEOOTHERMEX.COM
flow paths. This is because the amount of adsorption for the reactive tracer (theoretically) is proportional to the area of contact between the circulating fluid and the rock."

- "The choice of tracer is critical. The use of fluorescein as an inert tracer at Rosemanowes was convenient in many ways, but we had to make sure it was not changed chemically, nor significantly adsorbed in the reservoir. Are we so sure that this applies to fluorescein in the more vigorous conditions at Soultz? The idea that chemically reactive tracers, and tracers which are selectively adsorbed, can be used with impunity in high-temperature HDR systems worries me very much. Esters that hydrolyze to different extents at different temperatures are also sensitive to $\mathrm{pH}$, which may vary as significantly as temperature throughout a reservoir. The use of encapsulated tracers, in which the tracer is isolated chemically from the fluids, has been suggested, even to the extent of employing capsules, which dissolve selectively at different temperatures. Would the capsule's progress through the reservoir be impeded because its diameter was too large?

In spite of all this, a tracer is the only monitoring instrument which enters the reservoir, traverses the different flowpaths, and comes out the other side. Tester and his co-workers were quick to become aware of this in the early days at Los Alamos, and attention to improvements in tracer interpretation and choice of suitable tracer will be well worthwhile in the improvement of the overall design of HDR systems."

The simulator must include a tracer module, since tracers have become a significant tool for evaluating reservoirs. If thermally reactive tracers can be applied carefully in the field, the possibility exists to obtain information on cooling of the reservoir before it is observed at the production well.

\subsection{Two-Phase Flow}

Although not discussed in the HDR Structured Academic Review, the capability to model twophase flow will be required for most Enhanced Geothermal Systems, especially when 
TELEPHONE: $(510) 527-9876$

FAX: (510) 527-8164

E-MAIL MW Q GEOTHERMEXCOM

investigating the development of EGS projects adjacent to high-temperature hydrothermal reservoirs.

A complete EGS simulator will need to include two-phase flow and the consequent complexities of phase change, relative permeabilities, capillary pressure effects, etc.

\subsection{Summary of Simulator Features}

In addition to the capabilities required of a conventional geothermal reservoir simulator, namely, the ability to handle multi-phase fluid flow, heat transfer and tracer transport in porous or fractured media in three dimensions, the above discussion has identified desirable special characteristics of an EGS reservoir simulator. These desirable special characteristics are:

- explicit representation of fractures;

- the ability to change fracture opening as a function of effective stress;

- the ability to handle shear deformations and associated jacking of the fractures;

- a relationship between fracture aperture and fracture conductivity;

- channeling of flow in fractures;

- the ability to handle certain thermo-elastic effects; and

- the ability to handle mineral deposition and dissolution. 


\section{HDR GEOTHERMAL RESERVOIR SIMULATORS}

\subsection{Overview}

In this section we review simulators that have been applied to HDR geothermal systems. WillisRichards and Wallroth (1995) provide an extensive bibliography and a list of simulators available in 1995. Other reviews include those by Bodvarsson et al. (1986), Tsang (1987 and 1991), Pruess (1990) and Hudson (1995).

We have reviewed four simulators that are actively being applied to HDR reservoirs. These are:

- the "FRACTure" discrete-fracture simulator (Kohl and Hopkirk, 1995) with coupled hydro/thermal/mechanical processes in three dimensions, which has been used to model the Soultz reservoir using flow in a dominant fracture and a turbulent fluid model (Kohl, et al., 1997);

- the "GEOTH3D" simulator of Yamamoto et al. (1998), which uses microseismic data as a guide to the permeability distribution and has been applied to the Hijiori, Ogachi, and Fenton Hill reservoirs;

- the "FRACSIM-3D" code, a fracture network model that includes fluid flow and heat transfer (Jing, 1998), which has been used to model the Hijiori and Soultz reservoirs;

- "Geocrack2D," a simulator developed by Swenson and Hardeman (1997) that focuses on flow in fractures and has been used to model the Fenton Hill and Hijiori reservoirs.

\subsection{FRACTure}

\subsubsection{Overview}

FRACTure is a discrete-fracture, finite-element code for simulating the coupled hydraulic, thermal and mechanical behavior of fractured media (Kohl and Hopkirk, 1995). The model 
TELEPHONE: (510) 527-9876

FAX: $(510)$ 527-8164

E-MAIL:MW@GEOTHERMEX.COM

represents fluid flow through a permeable rock matrix, as well as through discrete fractures. The fluid flow may be modeled using both Darcian and turbulent governing equations.

Thermoelastic and poroelastic effects are applied to the porous media, and fracture openings are non-linearly linked to rock stress. Heat transfer includes conduction in the rock and transport in the fluid, and is coupled to the elastic and thermal solutions through thermal expansion and nonlinear constitutive relationships.

\subsubsection{Hydraulic Behavior}

Fluid flow is represented both in the rock matrix and in discrete fractures. Discrete fractures are treated as lower-dimensional elements that can be attached to higher-dimensional matrix elements. The flow may be represented with either a laminar or turbulent model. For the laminar model, conservation of mass is expressed using Darcy's Law as follows:

$$
S_{S} \frac{\partial P}{\partial t}=\nabla \cdot(K \cdot \nabla P)
$$

where $\nabla P$ is the pressure gradient, $K$ is the hydraulic conductivity (based on the cubic law), $S_{s}$ is the specific storage coefficient, and $t$ is time.

For situations where turbulent flow is suspected, FRACTure provides an alternate flow relationship:

$$
S_{S} \frac{\partial P}{\partial t}=\nabla \cdot\left(\bar{K}(\nabla P)^{1 / 2}\right)
$$

where

$$
\bar{K}=4 \log \left(\frac{1.9}{\varepsilon / D_{h}}\right)\left(\frac{a}{\rho}\right)^{1 / 2}
$$


TELEPHONE: (510) 527-9876

FAX: (510) 527-8164

E-MAIL:MW@GEOTHERMEX.COM

and $a$ is fracture aperture, $\varepsilon$ is the mean roughness, $D_{h}$ is the hydraulic diameter, and $\rho$ is the fluid density.

Fracture opening is controlled by the normal stress acting upon fracture and is described by a logarithmic joint law. The hydraulic aperture, $a_{h}$, is related to normal stress, $\sigma_{n}$, by:

$$
a_{h}=a_{h 0}+\left\{\left(\frac{\partial K_{n}}{\partial \sigma_{n}}\right) \ln \left(\frac{\sigma_{n 0}}{\sigma_{n}}\right)\right\}
$$

The behavior of the fracture is specified by a constant term $\left(\frac{\partial K_{n}}{\partial \sigma_{n}}\right)^{-1}$ which is called the stiffness characteristic of the fracture. This compliance relationship, coupled with the cubic law for fracture permeability, causes a highly non-linear effect that must be damped in the simulation in order to promote stability.

\subsubsection{Thermal Behavior}

Heat transfer calculations are carried out using heat conduction in the rock matrix and advection in the fractures. The heat transport in the fluid is iteratively coupled to the hydraulic simulation, and thermal conductivity properties are dependent on fluid pressure.

\subsubsection{Elastic Behavior}

In addition to the coupling between joint stress and the fluid pressure, stresses in the rock matrix are also affected by poroelastic and thermoelastic processes. The cooling of rock results in the creation of thermal stresses according to the following equation:

$$
S_{i i}^{T}=3 K \beta_{T} \Delta T
$$


where the $S_{i i}{ }^{T}$ are the thermal stresses, $\Delta T$ is the temperature change, $\beta_{t}$ is the coefficient of thermal expansion, and $K$ is the bulk modulus of the rock.

Fluid pressure also adds to the stress state of the rock, according to the following relationship:

$$
S_{i i}^{P}=\alpha_{P} \Delta P
$$

Where $\alpha_{B}$ is the Biot coefficient, and $\Delta P$ is the pressure change.

\subsubsection{Typical Application}

FRACTure has been used to model a variety of different geological problems, including:

- radon transport to buildings;

- space heating;

- tracer propagation;

- non-laminar hydraulic behavior at Soultz; and

- heat extraction during aquifer utilization.

Of relevance to this review, the model has also been used to compare simulations of HDR reservoirs using single and multiple fractures in both two and three dimensions. These applications are summarized below.

\section{Single 2-D Fracture Geometry}

As described by Kohl (1998), "This model was run to investigate the behavior of a single $200 \mathrm{~m}$ long, vertical fracture that connects directly the injection borehole with the extraction borehole .

... This example also serves to familiarize the reader with the complex output of such coupled 
TELEPHONE: (510) 527-9876

FAX: (510) 527-8164

E-MAIL:MW@GEOTHERMEX.COM

analyses. Injection takes place at the $\mathrm{x}-\mathrm{z}$ coordinates $(0,-5050)$ and production at $(0,-4950)$. A symmetry plane was assigned along the $\mathrm{z}$-axis at $\mathrm{x}=0 \mathrm{~m}$. In the $\mathrm{y}$-axis direction, the model used a unit depth of $1 \mathrm{~m}$ which simplifies the scaling-up of flow rates to more realistic situations and leaves the thermal or hydraulic field unaffected (i.e., the thermal drawdown). Model runs with fracture apertures varying between 50 and $200 \mu \mathrm{m}$ were performed."

"The results obtained for the 2-D single fracture geometry are illustrated in figure 6 . The temperature field after 30 years circulation is shown in the leftmost frame. The contrast between initial rock temperature and that of the injected fluid is $50^{\circ} \mathrm{C}$. It can be seen that the rock matrix undergoes significant cooling between the injection and extraction points along the vertical fracture. The cooling penetrates significantly into the host rock, the thermal drawdown some $50 \mathrm{~m}$ distance into the host rock amounting to $-10^{\circ} \mathrm{C}$ or $20 \%$ of the initial temperature contrast. The upper right frame indicates the temperature and flow history at the production point. After 30 years circulation the temperature drawdown has reached approximately $50 \%$. The dynamic behavior of the reservoir is highlighted by the produced flow rate, which varies due to the compliance of the fracture. The variation of the fracture aperture distribution due to changes in effective normal stress (shown in mid-right frame) is particularly strong near the injection point at $0,-5050 \mathrm{~m}$. This is also reflected by the stress perturbation in the host rock, close to the fracture (lower right frame), with maximum tensile stress perturbations at the injection point. Since the temperature perturbation penetrates deeper and deeper into the host rock, the thermal stress perturbation also increases with time. As a result, the predicted apertures after 30 years are considerably larger than those after 10 years of circulation. Thus, the system impedance after 30 years has decreased by approximately $20 \%$ from its initial value."

\section{Multiple 3-D Fracture Geometry}

Again, as described by Kohl (1998), "This model uses four different fracture sets and is based on the same discretization scheme as the 3-D single fracture model. Except the aperture distribution, identical parameter values were used for all fractures. A primary fracture 
corresponds to the former single-fracture model (described above). A second parallel fracture (also in the $\mathrm{y}-\mathrm{z}$ plane) is placed at $\mathrm{x}=80 \mathrm{~m}$ and is connected to the primary fracture by two orthogonal fractures. Identical initial apertures were assigned to the three latter fractures, which together form an indirect flow path between the wells. This indirect path becomes increasingly important as the impedance of the primary (direct) flow path is raised. This simple fracture network was discretized into a total of 112 Lagrange-type elements."

"The 3-D multiple-fracture simulation run results confirm the previous observations: depending on the choice for the initial aperture distribution, the temperature drawdown and the increase in flow rate with time is somewhat significant. Figure 7 shows the predicted temperature and aperture distributions for the case where the initial aperture is set to $100 \mu \mathrm{m}$ for the primary flow path, and $200 \mu \mathrm{m}$ for the three other fractures. Again, close to the injection point, the highest tensile stress perturbations and subsequently the largest fracture apertures develop. With the given parameter selection, the flow rate increases after 30 years to reach 2.5 times the initial value."

"The most important outcome of the various simulations is to demonstrate the large impact that dynamic behavior has on HDR performance during reservoir lifetime. Over periods of more than 10 years, the subsurface temperature field, the hydraulic flow field and the local stress field are subject to large changes. This behavior can only be modeled numerically. Thus it follows that only numerical simulations are able to give useful predictions for the long-term performance of HDR systems."

\subsubsection{Applicability to EGS Simulation}

This model lies in the category of discrete-fracture models. Its approach and concepts make it applicable to various analyses of reservoir operation. Its strength is the range of physics that has been implemented, with three-dimensional hydraulic, thermal and mechanical coupling. The code accommodates turbulent flow, but does not include two-phase flow or mineral deposition 
and dissolution effects. Channeling can be approximated by manually adjusting fracture element properties. There is no coupling between fracture shear displacement and aperture.

\subsection{GEOTH3D}

\subsubsection{Overview}

GEOTH3D (Yamamoto et al., 1995; 1997; 1998) uses a three-dimensional finite-difference approximation to solve for mass and energy balance based on Darcy's Law. The model can describe both water and heat transport in porous media. When applied to a geothermal reservoir, the available microseismic data is used to define non-uniform porosities in proportion to the microseismic intensity. Thus, the flow is greater in areas of the reservoir where the microseismic activity was most intense during stimulation.

\subsubsection{Hydraulic Behavior}

The following assumptions are used in the code:

- capillary pressure effects are neglected;

- water, steam, and rock are thermally equilibrated;

- the reservoir water may be single-phase or two-phase;

- relative permeability is a function of only the liquid volume saturation;

- viscosity is a function of pressure and temperature;

- porosity is a linear function of pressure; and

- the rock density, reservoir thickness, and intrinsic permeability tensor can be arbitrarily defined in three-dimensional space. 
Using Darcy's Law, the mass balance for water for both single- and two-phase conditions is used:

$$
\frac{\partial m}{\partial t}-\sum_{\alpha=1}^{2} \operatorname{div}\left\{\left(\frac{\rho}{\mu} k\right) \mathbf{K} \cdot \mathbf{g r a d} p-\left(\frac{\rho^{2}}{\mu} k\right) \mathbf{K} \cdot \mathbf{g}\right\}=q_{m}
$$

where:

$$
\begin{aligned}
& m=\phi \rho=\phi \sum_{\alpha=1}^{2} S_{\alpha} \rho_{\alpha} \\
& \phi=\phi_{0}+\beta_{r}\left(p-p_{0}\right) \\
& q_{m}=q_{m w}+q_{m s}
\end{aligned}
$$

and $\alpha=1$ and $\alpha=2$ correspond to water and steam phases, respectively, $\phi$ is the porosity, $\phi_{0}$ is the initial porosity, $\beta_{r}$ is the compressibility coefficient of the rock, $p$ is piezometric pressure, $p_{0}$ is initial phase pressure, $S$ is volumetric saturation, $\rho$ is the water density, $k$ is the relative permeability of the porous medium, $m$ is the total mass of the fluid, $t$ is the time, $\mathbf{K}$ is the intrinsic permeability tensor of the porous medium, $\mu$ is the dynamic viscosity, $q_{m}$ is the source term, and $\mathbf{g}$ is the gravitational constant vector. In the latest version of the code, only singlephase flow has been implemented (Eguchi, 1998a).

\subsubsection{Energy Balance}

The energy balance for water, steam, and rock is given by: 


\section{GeothermEx, Inc.}

TELEPHONE: (510) 527-9876

FAX: (510) 527-8164

E-MAIL: MW@GEOTHERMEX.COM

$$
\begin{aligned}
& \frac{\partial e}{\partial t}-\sum_{\alpha=1}^{2} \operatorname{div}\left\{\left(\frac{\rho}{\mu} k\right) \mathbf{K} \cdot \operatorname{grad} p-\left(\frac{\rho^{2}}{\mu} k\right) \mathbf{K} \cdot \mathbf{g}\right\}- \\
& \operatorname{div}\left\{K_{m}\left(\frac{\partial T}{\partial p}\right) \operatorname{grad} p+K_{m}\left(\frac{\partial T}{\partial h}\right) \operatorname{grad} h\right\}=q_{e}
\end{aligned}
$$

where:

$$
\begin{aligned}
& e=m h+(1-\phi) \rho_{r} h_{r} \\
& q_{e}=q_{m} h \\
& h=\frac{S_{w} \rho_{w} h_{w}+S_{r} \rho_{r} h_{r}}{\rho}
\end{aligned}
$$

and $r$ indicates rock, $K_{m}$ is the dispersion coefficient for heat conduction in the porous medium, $h$ is the enthalpy of the water-steam mixture, $h_{w}$ and $h_{s}$ are the specific enthalpies of water and steam, and $T$ is temperature. In each finite-difference cell, the water, steam, and rock are thermally equilibrated.

\subsubsection{Assignment of Permeability Using Acoustic Emission Data}

Based on measured microseismic measurements made during stimulation, non-uniform permeabilities are assigned to the rock volume. This assignment is made by converting the amplitude of the seismic signal to an energy value. On a block-by-block basis, energy values are calculated, and an averaging algorithm used. The energy values are converted to magnitudes, and finally, the magnitudes are converted to permeability values. Typically, the permeability will range over two orders of magnitude and will be divided into five ranges. 


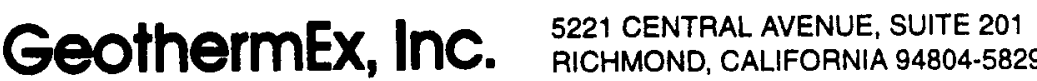

TELEPHONE: (510) 527-9876

FAX: (510) 527-8164

E-MAIL: MW@GEOTHERMEX.COM

\subsubsection{Typical Application}

A typical application is described by Eguchi et al. (1998a) for the Ogachi HDR reservoir (see Yamamoto et al., 1998, for an analysis of the Fenton Hill HDR site). The GEOTH3D code was applied to a thirty-day circulation test conducted at the Ogachi site in 1995. The measured pressures at the injection and production wells were used as boundary conditions and the resulting computed flow rates compared well with measured data. The model was then used to examine two alternate reservoir designs to improve recovery rates.

The first step in the analysis is to calculate the distribution of permeabilities in the reservoir based on microseismic data, as illustrated in figure 8 . The calculated pressure distribution near the injection and production wells at Ogachi is shown in figure 9. Figure 10 shows the flow in the reservoir. Finally, comparisons are made with the measured and predicted flow rates, as shown in figure 11.

\subsubsection{Applicability to EGS Simulation}

The model is appealing in its use of the microseismic data obtained during stimulation to make a non-uniform porous-medium model. However, the use of a porous medium is also a weakness. The model does not include discrete fractures, and, in general, porous-medium models err on the side of optimism with respect to energy production. This is because porous-medium models usually do not capture the sharp local temperature gradients and cooling that can occur in a fracture and do not represent changes in aperture due to stress or thermo-elastic effects.

\subsection{FRACSIM-3D}

\subsubsection{Overview}

FRACSIM-3D is a fracture network model described in the Ph.D. thesis of Jing (1998) and in Jing et al. (1998). This model is an extension of the 2-D fracture simulator FRACSIM-2D 
TELEPHONE: (510) 527-9876

FAX: (510) 527-8164

E-MAIL: MW@GEOTHERMEX.COM

(Willis-Richards et al., 1996). A similar model has been developed by Tezuka et al. (1998). The model focuses on the following reservoir effects:

- fracture shear and dilation during stimulation and circulation;

- themoelasticity during circulation; and

- chemical dissolution and precipitation during circulation.

The model can be used for analyzing both stimulation and reservoir testing operations, including tracer analysis and a simple chemical dissolution model. The steps in an analysis are discussed below.

\subsubsection{Generation of Fracture Network}

The model begins by generating a fractal fracture network, in which the fracture centers are uniformly random, the radius distribution fractal, and the orientation controlled by field data. Penny-shaped fractures are assumed. Initial fracture apertures are assumed to be proportional to the fracture radius. Figure 12 illustrates a typical fracture network.

\subsubsection{Hydro-Mechanical Behavior}

Fracture apertures are affected by the effective normal stress at the fracture surface and by the amount of shear displacement that determines the fit of the opposing rough surface. As pressure increases in the fracture, the effective stress is reduced and slip occurs using a friction law. The change in aperture during slip is a function of the shear displacement, as shown in figure 13.

\subsubsection{Fluid Circulation}

The fluid flow in the reservoir is calculated based on the fractal fracture model. In a typical model there may be 300,000 fractures, so the smeared approach is used to calculate an equivalent transmissivity to each block. Flow is assumed to be single-phase steady-state, and no further 
TELEPHONE: (510) 527-9876

FAX: (510) 527-8164

E-MAIL: MW@GEOTHERMEX.COM

shear of the fractures is considered. The flow is described using Darcy's Law, with the permeability based on the cubic law. Conservation of mass gives:

$$
K_{x} \frac{\partial^{2} P}{\partial x^{2}}+K_{y} \frac{\partial^{2} P}{\partial y^{2}}+K_{z} \frac{\partial^{2} P}{\partial z^{2}}=0
$$

where $K_{i}$ is the permeability based on the cubic law and $P$ is the pressure. The permeability between two elements is assumed to be the total sum of that from all of the fractures that intersect the dividing face, as shown in figure 14.

\subsubsection{Heat Extraction}

Heat extraction is calculated by assuming the temperature in the rock is equal to the fluid temperature. Heat flow is by conduction and convection of the fluid, given by:

$$
\begin{aligned}
& C_{R} \cdot \rho_{R} \cdot \frac{\partial T}{\partial t}+C_{W} \cdot \rho_{W} \cdot\left(Q_{x} \cdot \frac{\partial T}{\partial x}+Q_{y} \cdot \frac{\partial T}{\partial y}+Q_{z} \cdot \frac{\partial T}{\partial z}\right) \\
& =\lambda \cdot\left(\frac{\partial^{2} T}{\partial x^{2}}+\frac{\partial^{2} T}{\partial y^{2}}+\frac{\partial^{2} T}{\partial z^{2}}\right)
\end{aligned}
$$

where $T$ is the temperature of the rock and fluid, $t$ is the time, $\lambda$ is the thermal conductivity of the rock, $C_{R}$ and $C_{W}$ are the specific heat of the rock and the fluid, respectively, $\rho_{R}$ and $\rho_{W}$ are the density of the rock and fluid, respectively, and $Q_{x}, Q_{y}$, and $Q_{z}$ are the fluid velocities in the $\mathrm{x}, \mathrm{y}$, and $\mathrm{z}$ directions. The same discretization used for the flow is used for the heat transfer solution. That is, the temperature is smeared over the volume of each finite-difference volume. 
TELEPHONE: (510) 527-9876

FAX: (510) 527-8164

E-MAIL:MW@GEOTHERMEX.COM

\subsubsection{Chemical Water/Rock Interaction}

In an HDR reservoir, there is the possibility for dissolution and deposition of the rock minerals. This can increase the aperture in some regions and reduce the aperture in others. In the model, the dissolution rate is described by (Jing, 1998):

$$
\frac{\partial C}{\partial t}=\frac{S}{M} \cdot K(T) \cdot\left\{C^{\infty}(T)-C\right.
$$

where $C$ is the concentration of dissolved rock minerals, $t$ is time, $S$ is the interfacial surface area between rock and fluid, $M$ is the weight of fluid, $K(T)$ and $C^{\infty}(T)$ are the temperaturedependent reaction-rate constant and the saturation concentration, respectively. As for the flow model, the fractures that intersect each surface between the numerical solution blocks are identified and equivalent fractures created for which the aperture changes as a function of dissolution or deposition. When applied to Hijiori, the model did not predict a strong chemical effect.

\subsubsection{Typical Application}

The model has been applied to the Hijiori reservoir. First, the stimulation of the reservoir was modeled. A comparison of the predicted stimulation volume with the microseismic volume is shown in figure 15. The correlation between the measured and modeled volumes is quite good.

A statistical flow calculation was then performed. Depending on the generated fracture distribution, different flow rates between the injection and production wells were obtained; however, the mean values for the wells matched the observed flows quite well. Good matches were also obtained for the tracer calculations. The best-fit flow and tracer model was then used to predict reservoir behavior during a 30-day test and during long-term production and injection. For the 30-day test, the production temperature did not change significantly; the results for the long-term analysis are shown in figure 16. 


\subsubsection{Applicability to EGS Simulation}

This fracture network model can analyze both the enhancement of the reservoir (well stimulation) as well as the operation of the reservoir once it has been developed. The stimulation analysis appears to be quite strong, including shear dilation (based on a single global stress). Reported stimulation results show good correlation with the observed microseismic data. However, there is an active debate on the exact meaning of microseismic events, especially at Hijiori, where the best connections to the fracture system occur in relatively aseismic regions.

In evaluating reservoir operation, the mapping of fractures to form a non-uniform porousmedium model inevitably results in the previously discussed smearing of local gradients near a fracture and can lead to optimistic predictions of reservoir life. The inclusion of simple chemical dissolution and deposition is a fruitful area for further research.

\subsection{Geocrack2D}

\subsubsection{Overview}

The Geocrack2D finite-element code was developed to solve coupled thermal, hydraulic and mechanical problems where the flow is in fractures (Swenson, 1997a). A Geocrack2D model consists of rock blocks with nonlinear contact and discrete fluid paths between the blocks. Heat transfer occurs by conduction in the rock blocks and transport in the fluid. A tracer model is also included that uses particle tracking with thermal decay, diffusion, and adsorption of the tracer. The user interactively defines the finite-element mesh, the material properties, boundary conditions, and solution controls.

\subsubsection{Mechanical Behavior}

The continuum (rock) elements are derived following standard elasticity finite-element practice (Hughes, 1987). The assembled element contributions result in the global structural stiffness matrix $\mathbf{K}_{\mathrm{s}}$, the nodal displacement vector $\mathbf{u}$, and the global force vector $\mathbf{f}$, or: 
TELEPHONE: (510) 527-9876

FAX: (510) 527-8164

E-MAIL: MW@GEOTHERMEX.COM

$$
\mathbf{K}_{\mathrm{s}} \mathbf{u}=\mathbf{f}
$$

Interface elements are used to represent the nonlinear relationship between fracture opening and fracture stress. As such, they impose surface tractions on the continuum elements that are a function of the fracture opening. The "Bed-of-Nails" model (Gangi, 1978) is used to represent the relation between fracture opening and fracture stress:

$$
a=a_{o}\left[1-\left(\frac{\sigma}{\sigma_{c}}\right)^{m}\right]
$$

where $a$ is the fracture opening, $a_{o}$ is the zero-stress fracture opening, $\sigma$ is the effective stress, $\sigma_{c}$ is the stress at which the fracture is assumed to be closed, and $\mathrm{m}$ is a constant. In this model, as the fractures close, they become stiffer; as they open, they become softer.

\subsubsection{Fluid Flow}

The flow is assumed to be one-dimensional (planar) in a rock fracture. The fracture conductivity, $\mathrm{k}_{\mathrm{p}}$, is given by the cubic law:

$$
k_{p}=\frac{a^{3}}{12 \mu}
$$

where $\mathrm{a}$ is the fracture opening, and $\mu$ is the dynamic viscosity. The user specifies the initial opening at the equilibrium state, and then any displacements are added to that value when calculating the conductivity, $a=a_{0}+a_{\text {displacement }}$. The assembled finite-element global equations for the fluid flow model can be written as:

$$
\mathbf{K}_{\mathrm{p}} \mathbf{p}=\mathbf{q}-\mathbf{S} \frac{\partial \mathbf{a}}{\partial t}
$$


where $\mathbf{K}_{\mathrm{p}}$ is the global permeability matrix, $\mathbf{p}$ is the nodal pressure vector, $\mathbf{q}$ is the vector of specified flow rates, $\mathbf{S}$ is the fracture-opening storage matrix, and $\partial \mathbf{a} / \partial \mathrm{t}$ is the fracture-opening velocity vector. Including the storage term allows the solution of quasi-static transient problems, where the inertia of the fluid is neglected, but fluid is stored in the opening fractures.

\subsubsection{Heat-Transfer Model}

In the rock, heat transport is by conduction. The energy equation for transient, two-dimensional heat conduction is:

$$
k\left(\frac{\partial^{2} T}{\partial x^{2}}+\frac{\partial^{2} T}{\partial y^{2}}\right)=\rho c_{p} \frac{\partial T}{\partial t}
$$

where $T$ is the temperature, $t$ indicates time, $k$ is a constant value of conductivity, $\rho$ represents density, and $c_{p}$ is the specific heat.

The governing equation for transient, one-dimensional heat transfer including conduction, convection, and transport is:

$$
k \frac{\partial}{\partial x}\left(a \frac{\partial T}{\partial x}\right)-\rho v c_{p} \frac{\partial t}{\partial x}+h\left(T_{s 1}+T_{s 2}-2 T\right)=\rho a c_{p} \frac{\partial T}{\partial t}
$$

where $T$ is the fluid temperature, $T_{s}$ is the structure temperature at the bounding surfaces, $t$ represents time, $k$ is conductivity, $\rho$ is density, $v$ is volumetric flow rate, $c_{p}$ is the specific heat, and $h$ is the convection coefficient.

Using standard finite-element procedures, two sets of equations are obtained for the structure and fluid as:

$$
\left[\mathbf{K}_{\mathrm{S}}^{\text {th }}+\mathbf{H}_{\mathrm{S}}+\mathrm{C}_{\mathrm{S}}\right] \mathrm{T}_{\mathrm{S}}-\mathbf{H}_{\mathrm{S}} \mathrm{T}_{\mathrm{Fh}}=\mathrm{C}_{\mathrm{S}} \mathrm{T}_{\mathrm{S}}^{\mathrm{prev}}
$$


TELEPHONE: (510) 527-9876

FAX: (510) 527-8164

E-MAIL: MW@GEOTHERMEX.COM

$$
\left[K_{\mathrm{F}}^{\text {th }}+2 \mathbf{H}_{\mathrm{F}}+\mathrm{C}_{\mathrm{F}}+\mathbf{E}_{\mathrm{F}}\right] \mathbf{T}_{\mathrm{F}}-\mathbb{H}_{\mathrm{F}} \mathbf{T}_{\mathrm{Sh}}=\mathbf{C}_{\mathrm{F}} \mathbf{T}_{\mathrm{F}}^{\text {prev }}
$$

where $\mathbf{K}$ is the conductivity, $\mathbf{H}$ represents heat transfer between rock and fluid in the crack by a film coefficient, $\mathbf{C}$ is heat-capacity matrix, $\mathbf{T}$ is the vector of node temperatures, the sub-scripts $\mathrm{S}$ and $\mathrm{F}$ indicate structure (rock) and fluid, and the superscript "prev" indicates the values at the previous time step.

\subsubsection{Coupling of the Models}

The above derivations provide three sets of coupled equations. The coupling arises as follows:

- $\quad \mathbf{f}$, the load on the structure, includes loads on the rock blocks due to the fluid pressures in the joints, $\mathbf{p}$;

- $\mathbf{K}_{\mathrm{p}}$, the fluid-permeability matrix of the joints, depends on the cube of the joint openings, which are functions of the displacements, $\mathbf{u}$;

- $\partial \mathbf{a} / \partial t$, the joint-opening velocity, depends on $\mathbf{u}$ and the time step;

- the viscosity of the fluid is a function of the fluid temperatures;

- temperatures cause thermal strains (shrinkage in the structure); and

- the heat transport in the temperature solution includes the fluid flow rates calculated in the fluid solution.

Both pressures and displacements are solved simultaneously. This introduces coupling terms between the fluid and structure models that provide additional information to the solution and speed convergence of the problem. Analyses showing the effect of thermal deformation are given in Swenson and Hardeman (1997b). 


\subsubsection{Typical Application}

A typical application is an analysis of the Long-Term Circulation Test (LTCT) to be performed at the Hijiori reservoir (Swenson et al., 1999). The Geocrack2D model used to perform the analyses is shown in figure 17. This figure shows the rock blocks (rectangles), fractures/flow paths (blue paths), and well locations (circles and squares) in the model. The model represents a vertical section of the reservoir, extending from a depth of 1,475 to $2,475 \mathrm{~m}$. The horizontal extent is $1,000 \mathrm{~m}$, with the wells approximately centered within the model. The vertical section used for the model was chosen to bound the known volume of the reservoir. In the actual reservoir, the upper and lower fractures dip steeply. This 2-D representation can be viewed as section of the reservoir in which the fractures have been rotated to be horizontal. In the model, a uniform thickness (depth normal to the vertical plane of the model) of $50 \mathrm{~m}$ was used.

Typical results of the analysis are shown in figures 18 and 19. Figure 18 shows the local cooling that has occurred on the fractures. This illustrates how cool injection fluid can quickly arrive at the production point before uniform heat removal from the reservoir. Figure 19 shows the corresponding production temperatures from the fractures in the model. Again, the point is that some fractures can cool rapidly. This behavior has been observed at Hijiori using Pressure/Temperature/Spinner (PTS) logs (figure 20). As observed in the data, the lower fracture cooled from about $265^{\circ} \mathrm{C}$ to $225^{\circ} \mathrm{C}$ during 25 days of testing.

\subsubsection{Applicability to EGS Simulation}

The discrete-fracture approach used in Geocrack2D is similar to the approach in FRACTure. The fracture aperture is a function of effective stress, flow is calculated using the cubic law, thermo-elastic effects are included in the model, and tracers are calculated using a particletracking algorithm. The model does not include coupling of fracture aperture to shear displacement, and there is no porous-medium flow. The program is interactive, with graphical 
TELEPHONE: (510) 527-9876

FAX: $(510)$ 527-8164

E-MAIL: MW@GEOTHERMEX.COM

feedback to the user in all phases. At the present time the implementation is 2-D; however, a three-dimensional version is under development (Hardeman and Swenson, 1998). 


\section{HYDROTHERMAL GEOTHERMAL RESERVOIR SIMULATORS}

\subsection{Overview}

In this section we review simulators are being used to model hydrothermal reservoirs. The four simulators reviewed are:

- Lawrence Berkeley National Laboratory's "TOUGH2" simulator, which has been used extensively in hydrothermal reservoir simulation, nuclear waste isolation and groundwater modeling;

- the "TETRAD" simulator, developed by the Computer Modeling Group of Calgary, Alberta, which has been used extensively in hydrothermal, oil, and natural-gas reservoir simulation;

- the "STAR" simulator, developed by Maxwell Technologies of San Diego, California, which has been used for hydrothermal, oil, and natural-gas reservoir simulation (including heavy-oil thermal recovery); and

- the "FEHM" code, developed at Los Alamos National Laboratory, which has been used for simulation of hydrothermal, oil, and natural-gas reservoirs, nuclear-waste isolation, and groundwater modeling, as well as for the HDR reservoir at Fenton Hill reservoir (Zyvoloski et al., 1995; Bower, 1996).

\subsection{TOUGH2}

\subsubsection{Overview}

TOUGH2 is a general-purpose numerical simulation program for multi-phase, multi-component fluid and heat flow in porous and fractured media (Pruess, 1991), developed at Lawrence Berkeley National Laboratory. The space discretization is made directly from the integral form 
of the governing equations. This method avoids any reference to a global system of coordinates and allows irregular (non-orthogonal) discretization of the considered domain.

This code allows the simulation of 1-D, 2-D, and 3-D geometry of porous or fractured media. Heat and mass transfer processes are fully coupled. Tracer transport with adsorption and radioactive decay is accounted for. The treatment of gas in the code is extensive, with the inclusion of all the major gas species normally present in a geothermal reservoir. For dissolved solids, the effects of precipitation and dissolution of $\mathrm{NaCl}$ on porosity and permeability are included.

One of the more important features of TOUGH2 is the Multiple Interactive Continua or "MINC" method. In an EGS or HDR system, there normally exists a high temperature gradient between the host rock and the circulating fluid. MINC allows sequential partitioning of the rock matrix, and hence, the pressure and temperature transients between the host rock and the injected fluid can be simulated.

\subsubsection{Heat and Mass Transfer}

The mass and energy balance equations in TOUGH2 are written in the integral form:

$$
\frac{d}{d t} \int_{V_{n}} M^{(\kappa)} d V=\int_{\Gamma n} \mathbf{F}^{(\kappa)} \bullet \mathbf{n} d \Gamma+\int_{V_{n}} q^{(\kappa)} d V
$$

where $V$ is volume, $\Gamma$ is surface, and $\mathrm{t}$ is time. $M$ is the mass or energy per unit volume with $\mathrm{k}=$ $1, \ldots, \mathrm{NK}$ labeling the mass components, and $\mathrm{k}=\mathrm{NK}+1$ for the heat component. $\mathrm{F}$ is the massor heat-flux term, and $q$ is the mass- or heat-withdrawal term.

The above equation is then discretized in space using the "integral finite-difference" method (Edwards, 1972; Narasimhan and Witherspoon, 1976). This method avoids any direct reference 
to a global coordinate system; hence the discretized equations are valid for irregular sub-domains in one, two or three dimensions.

\subsubsection{Fluid Flow}

The mass-flux term in the governing equation is represented by Darcy's Law:

$$
\mathbf{F}_{\beta}=-k \frac{k_{r \beta}}{\mu_{\beta}} \rho_{\beta}\left(\nabla P_{\beta}-\rho_{\beta} \mathbf{g}\right)
$$

Where $k$ is the absolute permeability, $k_{r \beta}$ is the relative permeability of phase $\beta, \mu_{\beta}$ is the viscosity in phase $\beta$, and $P_{\beta}$ is the pressure in phase $\beta$.

In addition to Darcy's flow, TOUGH2 provides option for the treatment of diffusive fluxes of all components of all phases. The general expression for diffusive flux can be written as:

$$
\mathbf{f}_{\beta}^{\kappa}=-\phi \tau_{\beta} \rho_{\beta} \tau_{o} d_{\beta}^{\kappa} \nabla X_{\beta}^{\kappa}
$$

Where $\phi$ is the porosity, $\tau$ is the tortuosity, $\mathrm{d}$ is the diffusion coefficient, and $\nabla \mathrm{X}$ is the mass fraction gradient. The value of $\tau_{\beta}$ is saturation-dependent and is poorly known at present. In the spirit of conceptual consistency, TOUGH2 uses $\tau_{\beta}=k_{r}$.

\subsubsection{Solute Transport}

The newest version of TOUGH2 (V.2.0) provides the conventional non-reacting tracer package as well as a module for tracer transport with sorption and radioactive decay (Pruess, 1998).

Recent development of a new thermophysical properties module (Battistelli et al., 1997) allows for the accounting of the dissolution and deposition rate (chemically non-reactive and temperature/pressure dependent) of $\mathrm{NaCl}$ in the reservoir. This module will be included in 
TOUGH2 (V.2.0) as a standard feature. The simulator considers the rock as inert except for the single active mineral, $\mathrm{NaCl}$, which may be present in both aqueous solution and as a solid precipitate (Pruess 1998). From mass balances on $\mathrm{NaCl}$ in all phases, the volume of precipitated salt in the original pore space $\phi_{\mathrm{o}}$, which is termed "solid saturation" and denoted by $\mathrm{S}_{\mathrm{s}}$, is calculated. A fraction $\phi_{\mathrm{o}} \mathrm{S}_{\mathrm{s}}$ of the reservoir volume is occupied by precipitate, while the remaining void space $\phi\left(S_{s}\right)=\phi_{0}\left(1-S_{s}\right)$ is available for fluid phases. The reduction in pore space reduces the permeability of the medium.

The rapid increase in computer power has spurred the interest in combining a reactive-chemical transport module with a geothermal reservoir numerical simulator. Recent workers in this area are Lichtner (1992), Friedly and Rubin (1992), Steffel and Lasaga (1994), White (1995), and Lichtner and Seth (1996). Xu et al. (1998), and White et al. (1998) have linked a reactivechemical transport module to TOUGH2 and the preliminary results are promising.

An example is the recent work from Arihara (1999). In his study, the only chemically reactive species considered is silica $\left(\mathrm{SiO}_{2}\right)$. The conservation equation of silica in porous media can be expressed as:

$$
\nabla \bullet\left[\frac{k \rho_{w}}{\mu_{w}} C\left(\nabla P-\rho_{w} g \nabla Z\right)\right]-\nabla \bullet\left(\rho_{w} D \nabla C\right)=\frac{\partial}{\partial t}\left(\phi \rho_{w}\right)+q_{c}-R_{c} \phi
$$

where $C$ is the silica concentration, $D$ is the diffusion coefficient, $q_{c}$ is the rate of silica per unit volume, and $R_{c}$ is the reaction rate of silica.

Data obtained from the field appears to be in good agreement with the numerical simulation results using the above equation (Arihara 1999). 


\subsubsection{Multiple Interactive Continua (MINC)}

The use of a numerical simulator in predicting the behavior of a geothermal reservoir does not normally call for detailed description of the fluid flow at the single-fracture level; instead, the overall average permeability of a block of rock is usually sufficient in most geothermal applications. The interaction between the fractures and the host rock (matrix) is approximated using the dual-continua model of Warren and Root (1963). The formulation assumes that the interaction between the two is linearly dependent on the difference between the fracture pressure and the average matrix pressure. This assumption may be inadequate for some EGS systems where accurate calculation of heat transfer from the host rock requires the consideration of pressure and temperature transients between the fracture and matrix rock.

In TOUGH2, temperature and pressure transients between matrix rock and fractures can be accounted for using the Multiple Interactive Continua (MINC) method. This method allows the domain rock to be partitioned into fracture(s) and a series of matrix rock sub-domains, and the primary thermodynamic variables are rigorously calculated for each of the sub-domains. A schematic representation of MINC partitioning is shown in figure 21.

\subsubsection{Typical Application}

TOUGH2, and its predecessor TOUGH, is the simulator most widely used throughout the world for modeling hydrothermal reservoirs. An example of a TOUGH simulation is presented in figure 22, which shows the grid layout used to model the Cerro Prieto hydrothermal reservoir in Mexico (Antunez et al., 1991). This is a classic three-dimensional porous-medium model using a regular (orthogonal) grid. This model was calibrated against 20 years of production history from several dozen wells. Enthalpy of the producing wells and static pressures in a number of observation wells were used as the matching criteria.

An second example of a TOUGH2 model developed with non-orthogonal grid blocks is presented in figure 23, which shows the grid layout used to model the Zunil reservoir in 
TELEPHONE: (510) 527-9876

FAX: (510) 527-8164

E-MAIL: MW@GEOTHERMEX.COM

Guatemala (Menzies et al., 1991). In many TOUGH simulations, particularly for two-phase reservoirs, the matches to individual well data can be improved by subdividing the grid blocks with production wells. Figure 24 shows an example of this type of refinement for the Uenotai hydrothermal reservoir in northern Honshu, Japan (Antunez et al., 1990).

\subsubsection{Applicability to EGS Simulation}

TOUGH2 contains all the necessary features for the simulation of a typical geothermal reservoir. The MINC method provides a means for simulating the pressure and temperature transients between the fractures and matrix rock, enabling fracture networks to be effectively handled by TOUGH2. Discrete fractures can be easily handled as the simulator allows the grid to be highly irregular (non-orthogonal). An interface between TOUGH2 and Golder Associates' FracMan discrete fracture network generator has been developed, which allows FracMan to be used as a fracture mesh generator, while TOUGH2 is used as the solver. In this approach, individual fractures are modeled as a series of triangular, 2-D elements intersecting 3-D matrix grid blocks. Caution must be exercised when using a non-orthogonal grid, as the accuracy of the solutions depends upon the accuracy with which the various interface parameters in the flux equations can be expressed in terms of the average conditions in the grid blocks (Pruess, 1991).

Neither flow channeling nor fracture aperture change due to stress or thermo-elastic effects are accounted for in TOUGH2. Effects of pressure and temperature on porosity and permeability are simulated by the use of rock compressibility and expansitivity constant coefficients.

TOUGH2 enjoys a very wide base of users, which facilitates nearly continuous code development. Numerous add-on features suitable for EGS simulation have been developed, such as reactive-chemical transport (Xu et al., 1998, and White et al., 1998), and solid dissolution and precipitation and their effects on porosity and permeability (Pruess, 1998). Some of these features are going through the validation phase and are not included in the current version. 


\subsection{TETRAD}

\subsubsection{Overview}

TETRAD is a finite-difference numerical simulator first designed for use in oil and gas applications, and later modified for use in modeling geothermal reservoirs. Conservation equations are expressed in conventional differential equation forms and then discretized. These equations are fully coupled, and the simulator can be used to model 1-D, 2-D, and 3-D heat and mass flow in porous or fractured media. Fractures can be specified via the use of the doubleporosity/permeability option (see section 4.3.4). Each matrix or fracture block is assumed to be in local thermodynamic equilibrium. Interaction between the matrix and fractures is described using the formulation developed by Warren and Root (1963).

This simulator allows selective partitioning of the considered reservoir domain through the use of the "local grid refinement" option. This feature permits sections of the base grid to be partitioned, allowing selective portions of the simulated area to have higher grid block resolution. This local grid refinement is, however, not analogous to the MINC method described above (in the description of TOUGH2) and cannot be applied to model the pressure and temperature transients within a matrix block.

\subsubsection{Mechanical and Thermo-Elastic Behavior}

TETRAD does not have a direct correlation between fracture aperture and stress (shear or normal), nor does it have a relationship between fracture aperture and fracture conductivity. General rock deformation and its effects on rock porosity and permeability (due to changes in stress, pressure or temperature) are included in the simulator.

\subsubsection{Solute Transport}

This simulator provides a comprehensive package of chemically non-reactive tracers and noncondensible gases. Dissolved solids in the aqueous phase are assumed to be non-reactive, but the 
effects of the dissolved solids on the fluid thermodynamics equilibrium are accounted for. TETRAD has tracer options that allow radioactive and thermal decay, absorption, etc.

\subsubsection{Double Porosity}

TETRAD allows the considered domain to be partitioned into a dual-porosity/permeability system (fractures and matrix rock). Discrete fractures can be specified in the simulator, as long as they are oriented parallel to the base grid. Fracture width and spacing are given at the start of the simulation and remain independent of temperature or pressure. Porosity and permeability can be dependent on pressure and temperature by the use of constant rock compressibility and expansitivity coefficients.

\subsubsection{Typical Application}

TETRAD has been used to model many hydrothermal reservoirs. Figure 25 shows an example of a double-porosity, uniform-grid simulation model of the naturally fractured and seriously fluid-deficient reservoir at The Geysers, California (Menzies and Pham, 1995). Using TETRAD, production histories of several hundred wells over a 30-year period were used to calibrate this complex reservoir model. Figure 26 shows the match between the calculated and observed static pressures over a 10-year period for one of the observation wells.

Figure 27 shows an example of a discrete-fracture model of a hydrothermal reservoir (Beowawe, Nevada) developed with TETRAD (Butler et al., in press). The model was successfully calibrated against both the initial state of the system and more than 10 years of production history.

\subsubsection{Applicability to EGS Simulation}

This is considered to be a highly useful geothermal simulator that contains all the features necessary in hydrothermal reservoir studies. The non-reacting tracer package is comprehensive. Discrete fractures can be modeled, but aperture changes due to stress or thermo-elastic effects 
have not been included. Flow-channeling effects are not considered. Documentation is extensive, and TETRAD is considered one of the more user-friendly simulators in the industry.

\subsection{STAR}

\subsection{1 $\underline{\text { Overview }}$}

STAR is a hydrothermal reservoir simulator developed by Maxwell Technologies of San Diego, California. This simulator employs the finite-differencing scheme in the discretization of the governing equations. It is a 1-D, 2-D, or 3-D simulator and contains all the features commonly found in hydrothermal reservoir simulators, including a tracer module, deposition and dissolution of $\mathrm{NaCl}$, and non-condensible gases.

Standard treatment of rock compaction is included in the simulator with the use of a userprescribed rock-compressibility factor. Changes in pressure and temperature result in changes to rock porosity and permeability.

\subsubsection{Solute Transport}

Multiple tracers are included in the code. These tracers are in thermodynamic equilibrium with the reservoir fluid and are non-reactive. Salt dissolution and precipitation are included, and their effects on rock porosity and permeability are accounted for.

\subsubsection{Permeable Matrix}

STAR provides three different descriptions of the local fluid and heat flow in the rock. They are "porous media," "impermeable matrix" and "permeable matrix." Transient heat and mass flow between fractures and the matrix rock can be modeled using the "permeable matrix" option, while transient heat flow can be modeled using the "impermeable matrix" option. In each of these two options, the matrix blocks are represented by an equivalent spherical rock body subdivided computationally into concentric shells to represent the transient mass and/or heat 
flow (Pritchett, 1995). With this arrangement, the "permeable matrix" option can be considered equivalent to the MINC method (for regular, rectangular-grid arrangement).

\subsubsection{Production Options}

In addition to being coupled to a wellbore simulator, STAR has several surface "power-station" options to allow easier specification of wells in the field. Groups of production and injection wells can be allocated to "geothermal power stations" (incorporating separators, turbines, condensers, flash-tanks, etc.). Power-station operating constraints may be supplied by the user. Several power-station simulators (single-flash, double-flash, pressurized-injection, atmosphericinjection, condensate-injection, etc.) are available, as well as a generalized formula for unconventional systems (such as binary power plants). If desired, the simulator will automatically add make-up wells from time to time as required to maintain a specified steam rate (Pritchett, 1995).

\subsubsection{Typical Application}

STAR has been used to perform simulation studies in hydrothermal, natural-gas, and heavy-oil thermal-recovery projects (Pritchett, 1995).

\subsubsection{Applicability to EGS Simulation}

This is a typical reservoir simulator with all the necessary features for conducting hydrothermal reservoir simulation studies. The "permeable matrix" option can be used to model the pressure and temperature transients between fractures and matrix rock arranged in a rectangular grid system.

A comprehensive non-reacting tracer package is included in the simulator. Flow-channeling effects are not considered, nor are the effects of stress on fracture aperture. 
TELEPHONE: (510) 527-9876

FAX: (510) 527-8164

E-MAIL: MW@ GEOTHERMEX.COM

\subsection{FEHM}

\subsubsection{Overview}

The Finite-Element Heat- and Mass-Transfer Code (FEHM) is a program for the simulation of non-isothermal, multi-phase, multi-component flow in porous media (Dash et al., 1997; Zyvoloski et al., 1997). The equations of heat and mass transfer for multi-phase flow in porous and permeable media are solved in FEHM using the control-volume finite-element method. The permeability and porosity of the medium are allowed to depend on pressure and temperature. The code also has provisions for movable air and water phases and non-coupled tracers (that is, tracer solutions that do not affect the heat- and mass-transfer solutions). The tracers can be passive or reactive.

The code can simulate two-dimensional, two-dimensional radial, or three-dimensional geometries, and can handle coupled heat- and mass-transfer effects, such as boiling, dry-out, and condensation. The code is also capable of incorporating various adsorption mechanisms, ranging from simple linear relations to nonlinear isotherms.

Using either double-porosity/double-permeability or dual-porosity models, FEHM can simulate flow dominated by fracture flow. The dual-porosity method is appropriate when the fracture permeability controls the pressure communication in the reservoir and porous rock communicates only with the local fractures. The double-porosity/double-permeability model is appropriate when communication between the fracture and matrix blocks is needed in addition to the flow within the fracture and matrix blocks. The decision about which fracture model to use is often affected by the transient nature of the simulation. These alternative fracture formulations can be especially important for tracer-transport problems. 


\subsubsection{Mechanical Behavior}

The documented release version of FEHM does not include deformation of the rock. However, Bower (1996) implemented elastic behavior in a two-dimensional version of FEHM. She used a standard elasticity approach to incorporating this behavior.

\subsubsection{Fluid Flow}

FEHM includes fluid flow and heat conduction with the governing equation:

$$
-\bar{\nabla} \cdot(\mathbf{K} \nabla P)+q+\frac{\partial A_{e}}{\partial t}=0
$$

where $\mathbf{K}$ is an effective conductivity, $P$ is the pressure ( $\mathrm{T}$ or temperature for heat conduction), $A_{e}$ is the energy per unit volume, and $q$ is the specified energy source term.

\subsubsection{Heat and Mass Transfer}

FEHM also can simulate the transport of heat and mass within porous and permeable media.

The conservation equations for mass and heat transfer are respectively:

$$
\begin{aligned}
& -\bar{\nabla} \cdot\left(D_{m v} \bar{\nabla} P_{v}\right)-\bar{\nabla} \cdot\left(D_{m l} \bar{\nabla} P_{l}\right)+q_{m}+\frac{\partial}{\partial z} g\left(D_{m v} \rho_{v}+D_{m l} \rho_{l}\right)+ \\
& \frac{\partial A_{m}}{\partial t}=0
\end{aligned}
$$

and

$$
\begin{aligned}
& -\bar{\nabla} \cdot\left(D_{e v} \bar{\nabla} P_{v}\right)-\bar{\nabla} \cdot\left(D_{e l} \bar{\nabla} P_{l}\right)-\bar{\nabla} \cdot(K \bar{\nabla} T)+q_{e}+ \\
& \frac{\partial}{\partial z} g\left(D_{e v} \rho_{v}+D_{e l} \rho_{l}\right)+\frac{\partial A_{e}}{\partial t}=0
\end{aligned}
$$


TELEPHONE: (510) 527-9876

FAX: (510) 527-8164

E-MAIL:MW@GEOTHERMEX.COM

where $D$ is the transmissibility (including enthalpy as appropriate in the energy equation), $K$ is an effective thermal conductivity, $P$ is the pressure, $T$ is the temperature, $A_{m}$ is the mass per unit volume, $A_{e}$ is the energy per unit volume, $q$ represents the source and sink terms, $\rho$ is the density, $z$ is oriented in the direction of gravity, and $g$ represents the acceleration due to gravity. The subscripts $v$ and $l$ indicate quantities for the vapor phase and the liquid phase, respectively, and the subscripts $m$ and $e$ refer to mass and energy, respectively.

\subsubsection{Solute Transport}

FEHM can also simulate solute transport (including tracers) and allow for multiple, interacting solutes. The passive-solute equations are not directly coupled to the pressure field, but use the pressure field obtained by the heat- and mass-transfer solution. The transport equation for a given component is given by:

$$
\begin{aligned}
& -\bar{\nabla} \cdot\left(C_{\nu} D_{m v} \bar{\nabla} P_{\nu}\right)-\bar{\nabla} \cdot\left(C_{l} D_{m l} \bar{\nabla} P_{l}\right)-\bar{\nabla} \cdot\left(D_{c} \bar{\nabla} C_{l}\right)+q_{c}+ \\
& \frac{\partial}{\partial z} g\left(C_{\nu} D_{m v} \rho_{\nu}+C_{l} D_{m l} \rho_{l}\right)+\rho_{r} \frac{\partial C_{R}}{\partial t}+\frac{\partial A_{c}}{\partial t}=0
\end{aligned}
$$

where $C$ is the concentration of the solute, and $C_{R}$ represents the adsorption onto the porous media.

\subsubsection{Typical Application}

A typical application (and verification problem) is analysis of a 2-D areal reservoir with multiphase flow which was developed as part of the DOE Code Comparison Project (Molloy, 1980). The two-phase (water/water vapor), heat- and mass-transfer problem is characterized by a moving two-phase boundary. The modeled region has a cold fluid boundary that provides fluid to the system as discharge occurs through a well. Numerical difficulties can occur as nodes go from two-phase to compressed water. This problem is a good test for the two-phase routines, as well as the phase-change algorithm. 
TELEPHONE: (510) 527-9876

FAX: (510) 527-8164

E-MAIL:MW@GEOTHERMEX.COM

The geometry and boundary conditions are shown in figure 28 . Of particular note are the variable initial-temperature field and the prescribed pressure and temperature boundary.

Pritchett (1980) gives a more detailed discussion of this problem. The solution is verified by comparison of FEHM results to other codes. Figures 29 and 30 show comparisons with other solutions for this problem.

The Department of Energy has used FEHM in the Yucca Mountain Project for heat-flow, massflow and solute-transport modeling at a site scale, and for heat and mass flow at the canister scale. FEHM has also been used by DOE for simulations of multi-phase flow in oil and gas reservoirs.

A three-dimensional simulation of site-scale flow and radionuclide transport was completed for the Yucca Mountain unsaturated zone. The model was developed to a very high level of detail, including hydrostratigraphy, mineralogy, complex geologic structures, and radiochemistry. The simulation explicitly modeled the thermal/saturation effects of a repository design supporting different waste-package placement configurations.

\subsubsection{Applicability to EGS Simulation}

This model is a strong two-phase porous-medium model, including a double-porosity/doublepermeability capability. The model also has good tracer capabilities, with multiple reacting tracers. The 3-D version (which is the official release) does not include elastic deformation, discrete fractures, or aperture changes due to stress or thermo-elastic effects. These have only been included in the 2-D version (Bower, 1996) and have not been extensively used. The formulation is rigorous and well documented, with extensive verification.

FEHM can model movement of both water and steam phases and the movement of heat through convection and conduction, making it well-suited for EGS simulation. FEHM combines threedimensional volume elements with two-dimensional plate elements, allowing integration with discrete-fracture network (DFN) generators. As for TOUGH2, an interface between FEHM and 


\section{GeothermEx, Inc. 5221 CENTRAL AVENUE, SUTTE 201

TELEPHONE: (510) 527-9876

FAX: (5t0) 527-8164

E-MAIL:MW@GEOTHERMEX.COM

Golder's FracMan discrete fracture network generator has already been developed. FEHM does not provide mechanical coupling, but it does have tracer-test modeling interfaces, facilitating model calibration. 


\section{NUCLEAR-WASTE-ISOLATION SIMULATORS}

\subsection{Introduction}

The purpose of conducting this review of nuclear-waste-isolation simulators was to identify the unique features of these simulators that are not typically used in evaluating geothermal resources, but that may be applicable to EGS simulation problems. For each simulator, we provide an overview that describes the simulators in general terms, how hydraulic, thermal and elastic/mechanical behaviors are handled, examples of typical applications in waste-isolation problems, and a summary of the features that may be useful for EGS applications.

Portions of the code descriptions below are adapted from software documentation cited in references.

\subsection{FLAC and FLAC3D}

\subsubsection{Overview}

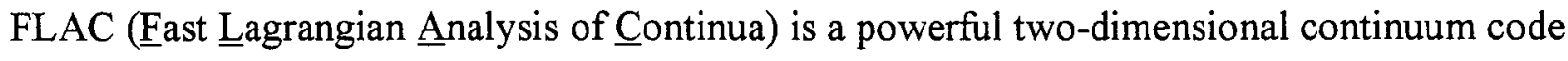
for modeling soil, rock and structural behavior (Board, 1989; Cundall, 1990). Based on research at the University of Minnesota, FLAC was developed by Itasca Consulting Group. Used interactively or in batch mode, FLAC is a general analysis and design tool for geotechnical, civil, and mining engineers that can be applied to a broad range of problems in engineering studies. The explicit finite-difference formulation of the code makes FLAC ideally suited for modeling geomechanical problems that consist of several stages, such as sequential excavation, backfilling and loading.

The formulation can accommodate large displacements, strains and non-linear material behavior, even if yield or failure occurs over a large area. FLAC is capable of simulating coupled thermal, hydraulic, and mechanical (T-H-M ) behavior of structures in soil, rock or other geological 


\section{GeothermEx, Inc.}

materials. The materials in FLAC are assumed to behave as a continuum and may undergo large-scale deformation due to plastic flow.

FLAC3D extends the capabilities of two-dimensional FLAC modeling to three dimensions. FLAC3D simulates the behavior of three-dimensional structures built of soil, rock or other materials that undergo plastic flow when their yield limits are reached. FLAC3D uses the same mixed-discretization scheme as FLAC to provide accurate modeling of plastic collapse and flow. Materials are represented by polyhedral elements within a three-dimensional grid that is adjusted by the user to fit the shape of the object to be modeled. Built-in primitive shapes allow the generation of a variety of complex geometries. With the graphics facilities in FLAC3D, highresolution, color-rendered plots are generated rapidly. A built-in graphics screen mode allows viewing of the model at any stage during creation or solution.

\subsubsection{Hydraulic Behavior}

FLAC solves isothermal flow through porous media according to Darcy's Law. Convective heat transport is not considered, but fluid density can be coupled to the local temperature field. Flow equations are solved by a variant of the point-relaxation technique.

Darcy's Law of flow through porous media is used in FLAC to describe the flow in terms of pressure, rather than head, given by:

$$
q_{i}=k_{i j}^{F} \frac{\partial P}{\partial x_{j}}
$$

and by the continuity equation at saturated grid points:

$$
\frac{\partial P}{\partial t}=-\frac{K_{w}}{n V}\left(\sum Q\right)
$$


where $q_{i}$ is the fluid-flux vector, $P$ is the pressure, $k_{i j}^{F}$ is the permeability tensor, $K_{w}$ is the bulk modulus of the fluid, $\mathrm{n}$ is the porosity, $\mathrm{V}$ is the volume associated with the grid point, and $\Sigma Q$ is the flow imbalance at the grid point. The coupled hydro-mechanical effects are basically the consolidation of the material due to interactions between the pressure and effective stress in the elements. The volume change due to mechanical deformation can then be considered.

\subsubsection{Thermal Behavior}

Both transient and steady-state heat transport and coupled thermo-mechanical effects can be handled in FLAC, expressed by volume change due to heat expansion and thermal stress changes of material due to heat flow. The heat transport is governed by Fourier's Law and the heat diffusion equation:

$$
\frac{\partial T}{\partial t}=\frac{1}{C_{p} \rho}\left(k_{x x}^{T} \frac{\partial^{2} T}{\partial x^{2}}+k_{y y}^{T} \frac{\partial^{2} T}{\partial y^{2}}\right)
$$

where $\mathrm{Q}_{\mathrm{i}}$ is the heat-flux vector, $\mathrm{T}$ is temperature $\left({ }^{\circ} \mathrm{C}\right), K_{i j}^{T}$ is the thermal-conductivity tensor and $\mathrm{C}_{\mathrm{P}}$ is the specific heat $(\mathrm{J} / \mathrm{kg}-\mathrm{K})$.

The stress change due to a change in temperature is given by:

$$
\Delta \sigma_{i j}=-\delta_{i j} 3 K \alpha \Delta T
$$

where $\mathrm{K}$ is the bulk modulus of the continuum, $\delta_{\mathrm{ij}}$ the Kronecker delta, $\alpha$ the linear thermal expansion coefficient, $\Delta \mathrm{T}$ the temperature change, and $\Delta \sigma_{\mathrm{ij}}$ the stress change.

Convective heat transfer due to fluid flow is not considered. 
TELEPHONE: $(510)$ 527-9876

FAX: $(510)$ 527-8164

E-MAIL: MW@ GEOTHERMEX.COM

\subsubsection{Elastic/Mechanical Behavior}

FLAC assumes that geologic materials behave as a continuum and may undergo large-scale deformation due to plastic flow. A grid of quadrilateral finite elements can deform in a largestrain mode with the deformation of elements (zones) with specific constitutive models of the materials. FLAC uses two sets of equations to handle deformation:

1) Cauchy's equations of motion for continuum bodies:

$$
\rho \frac{\partial \dot{u}_{i}}{\partial t}=\frac{\partial \sigma_{i j}}{\partial x_{j}}+\rho b_{i}
$$

where $\rho$ is the mass density of the material, $b_{i}$ is the body-force vector, $\sigma_{i j}$ is the stress tensor, $x_{j}$ is the coordinate vector, $u_{i}$ is the velocity vector, and $t$ is time; and

2) the constitutive equations of materials, in which the strain rate is expressed by velocity components as:

$$
\dot{\varepsilon}_{i j}=\frac{1}{2}\left(\frac{\partial \dot{u}_{i}}{\partial x_{j}}+\frac{\partial \dot{u}_{j}}{\partial x_{i}}\right)
$$

and the mechanical constitutive equation is given by:

$$
C\left(\sigma_{i j}, \dot{\sigma}_{i j}, \varepsilon_{i j}, \dot{\varepsilon}_{i j}, k\right)=0
$$

defined by users, where $k$ is a set of state variables and other material properties, depending on different laws. An incremental form of this equation is usually required for non-linear laws.

In FLAC's finite-difference scheme, the continuum is divided into a mesh of quadrilateral elements (zones). Internally, each quadrilateral element is subdivided into two overlaid sets of constant-strain triangle zones, termed $\mathrm{a}, \mathrm{b}, \mathrm{c}$ and $\mathrm{d}$ (see figure below). This scheme eliminates 
the modes of hourglass deformations, and a mixed-discretization scheme for isotropic and deviatoric components of stresses and strains can be used to avoid incompressibility condition of plastic flow.

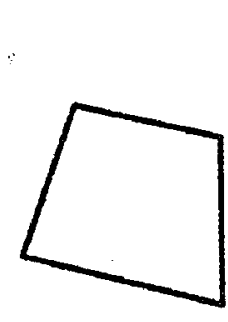

a)

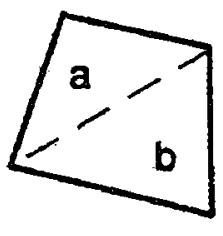

b)

\section{)}

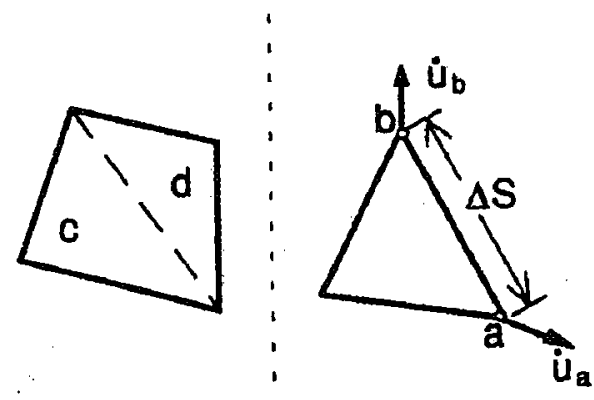

c)

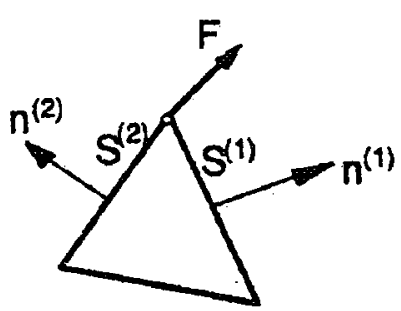

\section{Quadrilateral element (a), its subdivision into two overlaid triangle elements (b) and velocity and force vectors at grid points (c)}

Using the Gauss divergence theorem, the average derivative of a function, $f$, can be written as:

$$
\left\langle\frac{\partial f}{\partial x_{i}}\right\rangle=\frac{1}{A} \int_{\sigma} \frac{\partial f}{\partial x_{i}} d \sigma=\frac{1}{A} \int_{S} n_{i} f d s
$$

where $\mathrm{A}$ is the area of a plane domain $\sigma$, and $n_{\mathrm{i}}$ is the vector of the outward normal of the boundary $S$ of $\sigma$.

Let $\mathrm{f}$ be the nodal velocity components of a triangle element. Then the strain rates of the element can be expressed as:

$$
\frac{\partial \dot{u}_{i}}{\partial x_{j}} \cong \frac{1}{2 A} \sum_{k=1}^{3}\left(\dot{u}_{i}^{s}+\dot{u}_{i}^{e}\right)_{k} n_{j}(\Delta S)_{k}
$$


and the strain rates can be calculated. The principle is universal (i.e., it is not restricted to triangular elements).

The difference form of the equations of motion at one grid point is then written, using a centraldifference scheme:

$$
\dot{u}_{i}^{(t+\Delta t / 2)}=\dot{u}_{i}^{(t-\Delta t / 2)}+\left(\sum F_{i}\right) \frac{\Delta t}{m}
$$

where $F_{i}$ is the vector of all force terms, including gravity forces and applied loads contributed by all quadrilateral elements surrounding the grid point, and $\mathrm{m}$ is the lumped mass at the grid point. For large-strain problems, the new coordinates of the grid point are updated.

A central-difference scheme over the spatial grid is applied to the above equations, respectively within each time step. Other numerical techniques related to critical time step, damping, and calculation sequences can be found in related literature (Board, 1989; Cundall, 1990).

\subsubsection{Typical Application}

Projects in the field of waste isolation center on examination of the suitability of geologic materials for underground storage facilities. Because product storage is sometimes subject to adverse effects due to temperature and pressure in host rock, FLAC is of great use. FLAC is used to assess structural stability around storage facilities and is used in the analysis of earthquake-stress effects.

FLAC has been used in major waste-isolation programs in the Canada, France, Germany, Spain, Sweden, Switzerland, and the US. Users of FLAC include:

- ANDRA (French Nuclear Waste Disposal Agency);

- ENRESA (Spanish Nuclear Waste Disposal Agency); 
- NAGRA (Swiss Nuclear Waste Disposal Agency);

- POSIVA (Finnish Nuclear Waste Disposal Agency);

- $\quad$ NIREX (UK Nuclear Waste Disposal Agency);

- SKB (Swedish Nuclear Waste Disposal Agency);

- TRW (for the US DOE at Yucca Mountain)

- US Nuclear Regulatory Commission;

- URL-AECL (Canadian Nuclear Waste Disposal Research);

- Waste Isolation Pilot Project (WIPP), New Mexico (US DOE)

\subsubsection{Applicability to EGS Simulation}

FLAC's particular strength is modeling large strains in geologic materials. Unlike most stressstrain codes, the mixed-element Fast Lagrangian solution scheme in FLAC can successfully model plastic flow and failure deformations due to EGS development. As a result, FLAC is well suited for modeling EGS issues of subsidence and deformation. In addition, because FLAC includes capabilities for coupled T-H-M modeling, FLAC can be used for modeling wellbore stability and local wellbore stress-deformation-flow conditions.

\subsection{FracMan and MAFIC}

\subsubsection{Overview}

FracMan is a suite of codes developed by Golder Associates for three-dimensional discretefracture network (DFN) analysis. FracMan is available commercially from Golder Associates. 
FracMan is an integrated package of data-analysis, simulation, and visualization tools. The main components of FracMan are described below:

- FracSys provides tools for analysis of field data on orientation, size, shape, spatial structure and hydraulic properties for DFN model development.

- MAFIC provides single-phase (water or gas) flow and transport in single- and dualporosity (fracture/matrix) media. MAFIC includes advective and conductive heat transport, as well as solute transport.

- FracWorks generates stochastic and conditioned DFN models for a wide range of geologic settings including in sandstone, limestone/dolomite, siltstone, marl, and crystalline rocks.

- PAWorks and FraCluster provide detailed analyses of DFN pathways, networks, and rock blocks. PAWorks identifies transport pathways, and calculates transport along those pathways using a Laplace Transform Galerkin algorithm. FraCluster uses graph-theory searches to define hydraulic compartments and their properties.

- MASIC provides linear-elastic stress/strain analysis for fractured rocks based on the discrete-fracture network approach. MASIC uses the boundary-element approach based on fracture shear and normal stiffness.

\subsubsection{Hydraulic Behavior}

MAFIC (Matrix and Fracture Interaction $\underline{\text { Code) }}$ uses the Galerkin finite-element method to solve for flow and transport through DFN models. MAFIC simulates steady-state and transient flow, mass, and heat transport. Fractures are idealized using triangular finite elements. MAFIC provides for dual-permeability fracture/matrix interaction using quadrahedral finite elements, and dual-porosity behavior using a one-dimensional approximation based on the Warren and Root pseudo-steady-state approximation. 
TELEPHONE: $(510)$ 527-9876

FAX: (510) 527-8164

E-MAIL: MW@GEOTHERMEX.COM

MAFIC simulates solute transport and heat transport using a convective particle-tracking approach. Solute dispersion is simulated stochastically using orthogonal, normally distributed, lateral and transverse dispersion vectors. MAFIC solute transport includes matrix diffusion, mineral-specific retardation, and sorption features.

MAFIC was designed to simplify input-data requirements while providing maximum flexibility for the designation of boundary conditions. Input files may be specified by the user or generated by the FracMan DFN package. MAFIC is generally used for fracture networks of 10 to 10,000 fractures, although it has been applied for networks of up to 100,000 fractures using triangular finite elements and 300,000 fractures using pipe elements.

\subsubsection{Elastic/Mechanical Behavior}

FracMan models stress/strain using the boundary-element MASIC code, as well as POLY3D, available from Stanford University's Rock-Fracture Group. MASIC calculates mechanical response using boundary-element superposition of elastic half-space solutions for deformation of the rock matrix and for shear and normal deformation of fractures. MASIC works directly with MAFIC three-dimensional fracture networks.

\subsubsection{Typical Application}

FracMan and MAFIC have been used for flow and transport modeling in a wide range of projects, from mechanical analysis for civil construction projects to flow and radioactive-solute transport for radioactive-waste-repository projects, and for simulation of heat transfer due to steam flooding of oil reservoirs.

Example applications include:

- DFN simulation of heat and mass transport for improved oil recovery (IOR) by steam flooding at the Yates Field in West Texas; 
- DFN simulation of heat flow at the canister scale for radioactive waste disposal;

- DFN simulation of radionuclide transport at the kilometer scale for radioactive-wasterepository projects including Sellafield (UK), Äspö (Sweden), and JNC (Japan);

- large-scale DFN stress/strain analysis for glacial stress-relief displacements in Finland; and

- simulation of grout injection and foundation flowpaths for the Portuges Dam, Puerto Rico.

\subsubsection{Applicability to EGS Simulation}

Because of the ability to model the actual geometry of discrete conductors and flow barriers at a much greater level of realism and detail than is possible with conventional continuum approaches, FracMan's modeling approach is particularly well suited for EGS applications in which flow is controlled primarily by discrete fractures.

The primary advantage of FracMan for EGS simulation is that it combines the realism of geological and structural modeling (used to develop the DFN) with an array of application programs to facilitate simulation. MAFIC provides advective and conductive dual-porosity heat transport, and single-phase (water or gas) steady-state and transient flow and transport, which would be useful for modeling EGS well testing and development in single-phase hot-water reservoirs. MASIC provides stress analysis. PAWorks provides analysis of flow and transport pathways in DFN, potentially useful for EGS well-field design and resource management. FraCluster provides analysis of matrix-block properties, tributary drainage volume and compartmentalization for diagnosis of dual-porosity and hydraulic boundary effects.

FracMan uses loose rather than full coupling of T-H-M processes. As a result, each of the FracMan analyses are more stable and efficient and easier to interpret than fully coupled approaches. Loose coupling is achieved by iterating results between models. For example, the result of the flow code (MAFIC) is iterated into the stress code (MASIC), which calculates 
deformations. These deformations are then used to update fracture transmissivities, and the flow solution is re-run.

The FracMan approach can be improved for EGS applications by implementing options for fully integrated coupling, and also by improving the efficiency of its heat flow solution, which is based on particle tracking. In addition, the FracMan/FracWorks discrete-fracture generator can improve numerical EGS models by enabling the implementation of specific structural and geological features, which may exert considerable control on fluid flow.

\subsection{FTRANS}

\subsubsection{Overview}

FTRANS (Fractured flow and Transport of Radionuclides) was developed by the company GSI Geotrans for the US DOE. The goal of FTRANS is to combine flow and solute transport in porous media and discrete-fracture networks into a single, relatively easy-to-use code. FTRANS is a flow- and mass-transport-only code, but can be used with another DOE code, STEFAN, to link stress-strain results with the flow solution. FTRANS and STEFAN are both distributed by the US DOE National Energy Software Center.

\subsubsection{Hydraulic Behavior}

FTRANS is a two-dimensional finite-element code designed to simulate groundwater flow and transport of radioactive nuclides in a fractured porous medium. FTRANS takes into account fluid interactions between fractures and matrix blocks, advective-dispersive transport in the fractures and diffusion in the matrix blocks, and chain reactions of radionuclide components. It has the capability to model the fractured systems using either the dual-porosity or the discretefracture modeling approach, or a combination of both. 
TELEPHONE: (510) 527-9876

FAX: (510) 527-8164

E-MAIL: MW@GEOTHERMEX.COM

\subsubsection{Thermal Behavior}

FTRANS does not consider heat flow.

\subsubsection{Elastic/Mechanical Behavior}

FTRANS does not consider rock deformation. However, FTRANS can be used together with the US DOE elastic deformation code STEFAN to link hydraulic behavior with deformation.

STEFAN works with the same geologic model meshes as FTRANS, and has been coupled to FTRANS in applications for analysis of repository-waste-canister response.

\subsubsection{Typical Application}

FTRANS has typically been used in the US DOE for analysis of flow and transport in the vicinity of waste canisters and for large-scale flow and transport modeling. FTRANS is used for preliminary, rapid repository-performance assessment calculations because it is a 2-D code, and is therefore relatively rapid to set up and run, even though it includes full radionuclide decay, sorption, and diffusion features.

FTRANS has also been used on environmental projects for modeling of contaminant plume migration.

\subsubsection{Applicability to EGS Simulation}

FTRANS is a two-dimensional, single-phase fluid-flow and radionuclide-transport code. Its primary strengths for EGS are:

- the use of the finite-element method, which enables complex geological structures to be modeled;

- the integration of groundwater flow with solute transport; and 
- the use of a mixture of one- and two-dimensional elements to model fractures and rock matrix.

As a two-dimensional code, FTRANS is best suited for simple analyses that do not require consideration of three-dimensional geometries. FTRANS includes capabilities for chain decay and diffusive transport, which have little or no application to EGS simulation. FTRANS does not include either thermal or mechanical processes, and cannot be considered a true fracturemodeling code since the connectivity of fractures cannot be adequately represented in twodimensions.

\subsection{HYDREF, CHEF and VIPLEF}

\subsubsection{Overview}

HYDREF, CHEF and VIPLEF are three two-dimensional finite-element codes developed and applied by the Ecole des Mines de Paris (ENSMP) for ANDRA, the French radioactive-waste management agency.

HYDREF and CHEF use the finite-element method and integration in the time domain with an implicit scheme for the solution of two-dimensional transient or steady-state groundwater flow. A special joint element has been developed at ENSMP and incorporated into this code to consider DFN flow in two dimensions.

The code VIPLEF uses the finite-element method for the computation of displacements and stresses with either small or large strains. The constitutive behavior of the materials could be:

- linear or non-linear elasticity;

- elasto-plasticity with or without hardening (or softening);

- linear or non-linear visco-plasticity with or without hardening (or softening); and 
TELEPHONE: (510) 527-9876

FAX: (510) 527-8164

E-MAIL: MW@GEOTHERMEX.COM

- elasto-visco-plasticity with or without hardening (or softening).

The discontinuities are simulated by special joint elements. The analysis of the coupled T-M or $\mathrm{T}-\mathrm{H}-\mathrm{M}$ processes are performed by taking the results from separate thermal and hydraulic analyses (from HYDREF and CHEF) as input data to the code for mechanical analysis (VIPLEF).

\subsubsection{Hydraulic. Thermal and Elastic/Mechanical Behavior}

\section{General Functional Equations for Finite-element Formulation}

The finite-element formulations used in codes HYDREF, CHEF and VIPLEF are to minimize the following functional defined on a domain $\mathrm{D}$ with boundary $\mathrm{S}$ :

$$
\Psi(U)=\iiint_{D} k_{i j} \frac{\partial U}{\partial x_{i}} \frac{\partial U}{\partial x_{j}} d V-\iint_{\partial D_{Q}} Q U d S-\iiint_{D} q U d V
$$

In thermal (or hydraulic) analysis, $U$ is a scalar function of temperature (or hydraulic head), $Q$ is a surface flux, and $q$ is a volumetric source of heat (or fluid flow). In mechanical analysis, $U, Q$ and $q$ are vectorial functions of displacement, stress and volumetric force.

\section{Formulation of the Joint Element}

A special joint element is formulated in code HYDREF to consider the effects of variations in temperature and aperture of the discontinuities. Density and viscosity of the water vary with temperature. The permeability of the discontinuities used in this code is given by:

$$
k=\frac{e^{3} \rho_{0} g}{12 \mu_{0}}\left[1-\beta\left(T-T_{0}\right)\right]\left|1+v\left(T-T_{0}\right)\right|
$$

where $T$ and $T_{0}$ are the current and initial temperature, $\rho_{0}$ and $\mu_{0}$ are the initial density and viscosity of the water, and $\beta$ and $v$ are constants. 
FAX: (510) 527-8164

E-MAIL:MW@GEOTHERMEX.COM

The joint element is a six-noded line element of two sides (each side has three nodal points, as shown in the figure below). A quadratic variation over the element length (L) is assumed for the hydraulic head $(\mathrm{H})$, temperature $(\mathrm{T})$ and joint aperture $(\mathrm{e})$.

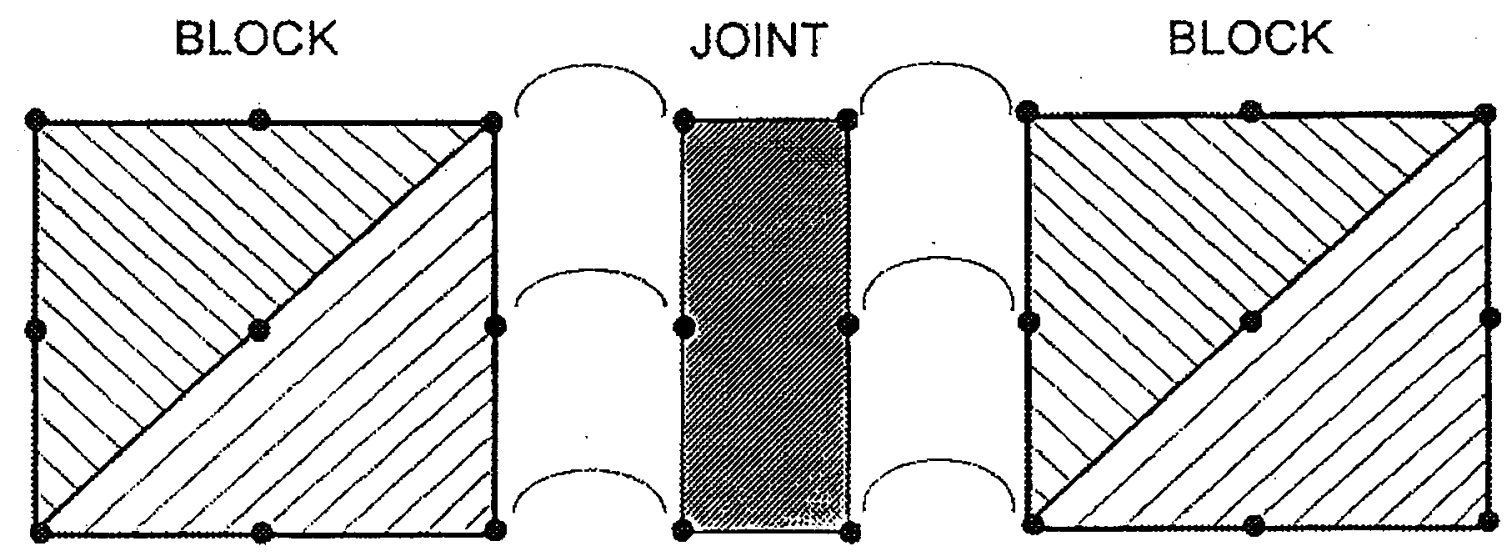

Elements of rock matrix and joint in code HYDREF and VIPLEF

\section{T-H-M Coupling Logic}

The coupled T-H-M process is simulated by combined applications of the three codes. The nodal temperature is calculated first with $\mathrm{CHEF}$, followed by a mechanical calculation for displacements due to thermal loading with VIPLEF, but without fluid flow. Then the apertures of joints, hydraulic heads and pressures at nodes are calculated with HYDREF. For next step, VIPLEF and HYDREF are used alternatively and iteratively to calculate the displacements, apertures and pressures in the joints until a stable solution is obtained.

\subsubsection{Typical Application}

These three codes have been used by ENSMP (Ecole des Mines de Paris) for the DECOVALEX project to simulate coupled heat and mass flow and deformation in heated-block experiments. DECOVALEX (DEvelopment of COupled models and their VALidation against EXperiments) is an international co-operative project to support the development and validation of coupled 
FAX: (510) 527-8164

E-MAIL: MW@GEOTHERMEX.COM

T-H-M processes. The project started in 1991 and is still active as of 1999 . The current project participants include the Commission of European Communities (CEC) and radioactive-waste organizations from Canada, Japan, Sweden, Finland, France, the UK, and the US. The project defines a series of test cases based on experimental sites at which extensive T-H-M test data are available, and it provides these test cases to project participants for modeling. DECOVALEX cases have included 1-m-scale heated-block experiments, 10-m-scale mine models, and 100-mscale shaft models in fractured and heterogeneous rocks.

The HYDREF, CHEF, AND VIPLEF codes were assembled specially for the DECOVALEX project and we do not know of any other applications in which they have been used.

\subsubsection{Applicability to EGS Simulation}

These three two-dimensional finite-element codes can be combined to model T-H-M effects at the scale of individual discontinuities. This has significant potential usefulness for EGS analyses of fractured reservoirs. The codes require user specification of the governing equations for heat and mass flow and stress-strain, and are therefore not well suited for general use. However, where specific constitutive equations are to be used, these codes provide a loosely coupled alternative to the PHOENICS code (see below) for EGS simulation. These codes would require extensive development for user-interfaces and coupling approaches to be widely applicable for EGS simulation.

\subsection{MAGNUM2D}

\subsubsection{Overview}

The MAGNUM2D code was developed by Battelle Pacific Northwest Laboratories (BPNL) for the US DOE to provide a single platform for transient and steady-state analysis of coupled heatand mass-flow processes. MAGNUM2D couples groundwater flow, heat conduction, and advective heat transport by using a single finite-element solver to solve the partial differential 
TELEPHONE: (510) 527-9876

FAX: (510) 527-8164

E-MAIL: MW@GEOTHERMEX.COM

equations of heat and mass flow, iterating between the solutions to model the resulting non-linear behaviors. MAGNUM2D's special strength is its coupling of fluid density to temperature, facilitating simulation of buoyancy driven flows.

\subsubsection{Hydraulic Behavior}

MAGNUM2D solves dual-permeability heat and mass flow for fractured rock using the finiteelement method. MAGNUM2D uses line elements for the 2-D DFN model, together with triangular and quadrilateral elements to represent the rock matrix. MAGNUM2D solves singlephase flow only.

MAGNUM2D solves the standard continuity equation for both heat and mass flow using Galerkin finite-element solver. Solver efficiency is improved by using the block-diagonal frontal-solution technique. MAGNUM2D formulates the finite-element equations in terms of incremental changes in the dependent variables using a Newton-Raphson approach. This facilitates modeling of non-linear behaviors such as changes in permeability with temperature and density driven flow. MAGNUM2D can solve porous-medium flow with anisotropic material properties.

MAGNUM2D can solve steady-state and transient flow in both 2-D planar or 2-D radial coordinate systems. 2-D radial coordinates are particularly useful for simulating flow around wellbores.

\subsubsection{Thermal Behavior}

MAGNUM2D solves heat flow using the same finite element solver implemented for mass flow. The coupled equations of heat flow and mass flow are solved by iteration. 


\subsubsection{Elastic/Mechanical Behavior}

MAGNUM2D does not solve for rock mass deformation and mechanical behavior. However, as part of the iterative coupling between the finite-element solutions for heat and mass flow, MAGNUM2D calculates the thermo-elastic stress on 1-D fracture elements. MAGNUM2D can then use these thermo-elastic stresses to recalculate fracture aperture and hence fracture transmissivity.

\subsubsection{Typical Application}

MAGNUM2D was developed for US DOE's Basalt Waste-Isolation Project (BWIP), and was used in that project to analyze thermally driven mass flow in the deep basalts beneath the Pasco Basin at the Hanford, Washington site. These simulations of non-isothermal groundwater flow assumed that the basalt could be represented as a heterogeneous anisotropic medium. Applications of MAGNUM2D outside of the BWIP project have not been identified.

\subsubsection{Applicability to EGS Simulation}

The strength of MAGNUM2D lies in its ability to model heat and mass flow in combinations of discrete fractures and matrix within a single code. These range from pure DFN approaches to dual-porosity and continuum approaches. However, since these are implemented only in two dimensions, they are limited in applicability to relatively simple problems. MAGNUM2D does consider coupling of fracture aperture due to thermo-mechanical stresses, and allows for complex thermal loadings.

\section{$5.7 \underline{\text { MOTIF }}$}

\subsubsection{Overview}

MOTIF was developed by Atomic Energy Canada Limited (AECL) for the Canadian radioactive waste management project, and was used by AECL as part of the DECOVALEX project. 
MOTIF uses the finite-element method to solve flow and transport problems in porous media in three-dimensions. MOTIF features include transient and steady-state groundwater flow, heattransfer and quasi-static T-H-M processes.

\subsubsection{Hydraulic Behavior}

MOTIF is a dual-porosity, dual-permeability flow code. The solid matrix is represented by eightnoded hexahedral elements. Fractures are represented by four-noded planar elements for flowand heat-transport calculations, and by eight-noded planar joint elements for stress analysis. MOTIF uses Galerkin finite elements to solve single-phase groundwater flow equations, as well as heat and equilibrium equations. For transient-flow problems, MOTIF uses the finitedifference method for time-stepping in the time domain.

MOTIF solves single-phase water flow. MOTIF uses a modified form of Biot's equation for flow through deformable porous media.

\subsubsection{Thermal/Mechanical Behavior}

MOTIF models thermal/mechanical behavior by applying the finite-element method to solve heat flow and thermo-elastic deformation in the same finite-element model used for groundwater flow. MOTIF iterates between groundwater flow, heat flow, and deformation using Picard iteration to solve the coupled problem.

The general three-dimensional T-H-M equilibrium equation solved by MOTIF is based on an extension of Biot's solution for anisotropic poro-elastic materials (Biot, 1941), as given by:

$$
\begin{aligned}
& \frac{\partial \sigma_{i j}}{\partial x_{j}}+b_{i}=0 \\
& \sigma_{i j}=C_{i j k l} \varepsilon_{k l}-\left(P-P^{0}\right) U_{i j}^{*}-\left(T-T^{0}\right) W_{i j}^{*}+\sigma_{i j}^{0}
\end{aligned}
$$


TELEPHONE: (510) 527-9876

FAX: (510) 527-8164

E-MAIL:MW@GEOTHERMEX.COM

$$
\begin{aligned}
& \varepsilon_{i j}=\frac{1}{2}\left(\frac{\partial u_{i}}{\partial x_{j}}+\frac{\partial u_{j}}{\partial x_{i}}\right) \\
& W_{i j}^{*}=C_{i j k l} W_{k l} \\
& U_{i j}^{*}=C_{i j k l} U_{k l}
\end{aligned}
$$

where: $i, j, k, 1=1,2,3 ; \varepsilon_{i j}$ and $\sigma_{i j}$ are strain and stress tensors, respectively; $P$ and $T$ are pore pressure and temperature with their respective initial values being $\mathrm{P}^{0}$ and $\mathrm{T}^{0} ; \mathrm{U}_{\mathrm{I}}$ is the displacement vector; $C_{i j k l}$ is the elasticity-constant tensor; $U_{i j}$ and $W_{i j}$ are the isothermal hydroelastic-constants tensor and thermoelastic-constants tensor, respectively; $b_{i}$ is the bodyforce vector and $\sigma_{\mathrm{ij}}^{0}$ is the initial-stress tensor.

Motif uses a hyperbolic function to relate the normal stress on fractures to their normal displacement, which in turn controls aperture and couples fracture transmissivity:

$$
\sigma_{n}=\frac{v_{m} k_{m}(\Delta v)}{v_{m}-\Delta v}
$$

where: $\Delta \mathrm{v}$ is the displacement normal to the discontinuity plane; $k_{m}$ is the initial normal stiffness of the discontinuity; $v_{m}$ is the maximum closure; and $\sigma_{n}$ is the normal stress.

The shear-stress/shear-displacement relation is based on a simplified (linearized) Barton-Bandis model and is given by (Barton and Choubey, 1977):

$$
\begin{aligned}
& \tau=\frac{\tau_{p}}{u_{p}} u_{s}, \quad\left|u_{s}\right| \leq\left|u_{p}\right| \\
& \tau=\tau_{p}+\frac{\tau_{r}-\tau_{p}}{u_{r}-u_{p}}\left(u_{s}-u_{p}\right), \quad\left|u_{p}\right| \leq\left|u_{s}\right| \leq\left|u_{r}\right|
\end{aligned}
$$


TELEPHONE: (510) $527-9876$

FAX: (510) 527-8164

E-MAIL: MW@GEOTHERMEX.COM

$$
\begin{aligned}
& \tau=\tau_{r}, \quad\left|u_{s}\right| \geq\left|u_{r}\right| \\
& \tau_{p}=\sigma_{n} \tan \left[\phi_{r}+i\right]=\sigma_{n} \tan \left[\phi_{r}+J R C \log _{10}\left(J C S / \sigma_{n}\right)\right] \\
& u_{p}=A(J R C)^{B} \\
& u_{r}=m\left(u_{p}\right) \\
& \tau_{p}=\left(\frac{0.5+r}{1.0+r}\right) \tau_{p}
\end{aligned}
$$

where: $u_{s}$ is the current shear displacement; $u_{p}$ is the shear displacement corresponding to when the peak shear stress $\left(\tau_{r}\right.$ ) first occurs; $u_{r}$ is the residual displacement; $i$ is the effective dilation angle of the discontinuity surface; $r$ is the ratio $\phi_{\mathrm{r}} / \mathrm{i}$, and $\mathrm{A}, \mathrm{B}$, and $\mathrm{m}$ are empirical constants.

\subsubsection{Typical Application}

MOTIF was used extensively by AECL for repository-performance assessment, for simulation of flow, transport and deformation at AECL's Underground Research Laboratory (URL) in Manitoba, where it was used successfully to model coupled groundwater flow and deformation during construction of the Lab's access shaft. MOTIF was used by AECL for simulation of heated-block experiments as part of the DECOVALEX project, simulating the effect of coupled heat and mass flow and deformation. We are not aware of any application of MOTIF outside of the Canadian radioactive waste program.

\subsubsection{Applicability to EGS Simulation}

MOTIF provides an integrated platform for coupled heat- and mass-flow and transport modeling, combined with systems analysis for optimization and risk assessment. The systems analysis capabilities are somewhat attractive for consideration of design alternatives and geological risks. 
FAX: (510) 527-8164

E-MAIL:MW@GEOTHERMEX.COM

However, the simulator's complexity makes it relatively difficult to use for EGS applications, particularly for those not familiar with systems analysis approaches.

\subsection{NAPSAC}

\subsubsection{Overview}

NAPSAC is AEA Technologies' discrete-fracture network (DFN) flow and transport model. Like NAMMU, NAPSAC is available for commercial licensing. NAPSAC uses an innovative mixed finite-element/influence-function approach that provides a high level of efficiency for steady-state flow solutions, and also facilitates modeling of the effect of heterogeneous aperture distributions on fracture surfaces.

\subsubsection{Hydraulic Behavior}

NAPSAC solves steady-state and transient flow by a mixed finite-element/influence-function approach. This algorithm makes it possible for NAPSAC to model on the order of $10^{5}$ fractures in steady-state simulations. NAPSAC uses a particle-tracking algorithm for solute transport, and does not provide heat-flow modeling capabilities. NAPSAC is a single-porosity/singlepermeability model, but can be coupled with NAMMU to provide dual-porosity or dualpermeability capabilities. NAPSAC is a single-phase (water-only) flow code.

NAPSAC's fracture generation is based upon the use of rectangular elements, which can be generated either stochastically or deterministically.

\subsubsection{Thermal Behavior}

Heat transport is not considered in NAPSAC. 
TELEPHONE: (510) 527-9876

FAX: (510) 527-8164

E-MAIL: MW@GEOTHERMEX.COM

\subsubsection{Elastic/Mechanical Behavior}

NAPSAC does not model rock mass mechanical response, but does provide for coupling between fracture transmissivity and changes to normal stress, through changes in the fracture aperture. The approximation to the normal stress may be found from an analytical solution or an empirical specification of the stress field derived from experimental observations. The flow through the modified fracture network can be calculated to assess the effect of stress on the flow field. The effect of changes in pore pressure on the mechanical properties of the network is not modeled by NAPSAC.

\subsubsection{Typical Application}

NAPSAC is typically used for flow and solute-transport modeling on scales ranging from 10 to $200 \mathrm{~m}$. It has been used for groundwater flow and solute-transport modeling of the Sellafield radioactive-waste-repository site in the UK, the Äspö Hard Rock Laboratory underground site in Sweden, and the Grimsel Underground Rock Laboratory, operated by the Swiss agency Nagra. NAPSAC has also been used for modeling flow in oil reservoirs, and for investigation of solute transport at contaminated land sites.

\subsubsection{Applicability to EGS Simulation}

NAPSAC is a discrete-fracture network (DFN) mass-flow modeling code, widely used for flow and transport modeling for radioactive-waste-disposal applications. NAPSAC uses a mixed finite-element/influence-function approach, making it more efficient than FracMan/MAFIC for steady-state problems, but less efficient for transient problems. NAPSAC has commercialquality user interface and post-processing capabilities, and it has been integrated with NAMMU for multi-scale modeling applications. NAPSAC does not provide heat-flow modeling capabilities. NAPSAC has been used to model fracture data from the British Hot Dry Rock project at Rosemanowes. 


\subsection{ROCMAS}

\subsubsection{Overview}

ROCMAS is a 2-D finite-element code developed at Lawrence Berkeley National Laboratory for solution of two-dimensional problems of coupled T-H-M processes in geological systems. ROCMAS solves transient coupled thermal, hydraulic, mechanical and transport problems in saturated and unsaturated geological media. ROCMAS includes both porous media and discretefracture (DFN) elements for both flow and mechanics.

A three-dimensional version of ROCMAS has also recently been developed. A specially developed algorithm for plastic failure in a preferred direction has been implemented into this three-dimensional code. This implies that the code can simulate failures that take place along preferable directions that represent the existing joint sets in the rock mass.

\subsubsection{Hydraulic Behavior}

ROCMAS uses the finite-element method to solve steady-state and transient, single-phase, single-porosity/dual-permeability groundwater flow and heat flow, with coupled stress/strain analysis. ROCMAS solves single-phase flow, based on Darcy's Law (Laminar flow). In this model, continuum elements are represented by triangular elements, and fractures are represented by one-dimensional line elements, with transmissivity based on the cubic law for parallel plates.

\subsubsection{Thermal Behavior}

ROCMAS solves conductive heat transport by the finite-element method. Temperature fields are coupled to hydraulic properties through the use of thermo-elastic stresses in the elastic/mechanical analysis. 
TELEPHONE: $(510)$ 527-9876

FAX: (510) 527-8164

E-MAIL: MW@GEOTHERMEX.COM

\subsubsection{Elastic/Mechanical Behavior}

ROCMAS calculates stress and strain through a combination of elastic solutions for the rock mass and special four-noded, strain softening joint elements for fractures. The changes in fracture aperture due to changes in mechanical and thermoelastic stresses are coupled to the flow solution through the use of a parallel-plate cubic law for fracture transmissivity.

The fracture mechanical model is based upon the combination of Goodman's joint model for stress-strain normal to fractures (Goodman, 1976) and a Ladanyi and Archambault criterion (Lardanyi and Archambault, 1970) for shear displacement for stress-strain shear to fractures.

\subsubsection{Typical Application}

ROCMAS was used by Lawrence Berkeley National Laboratory (LBNL) during the DECOVALEX project to model coupled flow and fracture deformation for simulation of heatedblock experiments at the 1-m scale. ROCMAS is not widely distributed or applied.

\subsubsection{Applicability to EGS Simulation}

ROCMAS was developed specifically to model heat and mass flow together with rock mass deformation for simulation of thermal effects at scales of meters to tens of meters. As a result, ROCMAS can be used directly as a T-H-M process simulator, and therefore could be applied EGS simulation.

ROCMAS is primarily a 2-D code, with fractures implemented as line elements. However, a 3-D version has recently become available. ROCMAS is designed for small-strain problems, but has been adapted for larger strains and plastic deformation, which might ultimately make it possible to use the code for simulation of well stimulations. 


\section{$5.10 \underline{\text { SWIFT } 98}$}

\subsubsection{Overview}

SWIFT98 (ㅁandia Waste Isolation Flow and Transport) is a fully transient, three-dimensional simulator for the flow and transport of fluid, heat (energy), brine, and radionuclide chains in porous and fractured geologic media. SWIFT was originally developed for the US DOE Sandia National Laboratory, and early versions are available for public distribution. More recent, fully tested versions with modern pre- and post-processing are available commercially, e.g., from Scientific Software.

\subsubsection{Hydraulic and Thermal Behavior}

SWIFT98 uses the finite-difference method to solve for transport of fluid, heat, and brine, coupled with fluid density, fluid viscosity, and porosity. Steady-state options are available for the fluid and brine equations, and both Cartesian and cylindrical coordinate systems may be used. Both dual-porosity and discrete-fracture conceptualizations may be considered for the fractured media.

SWIFT solves porous media using a global, three-dimensional finite-difference algorithm, discretized in space and time. For fractured media, the three-dimensional finite-element algorithm is used for the fractures, a local (one-dimensional) process simulator is used for the rock matrix. . Migration within the rock matrix is characterized as a one-dimensional process.

The SWIFT finite-difference solver provides a number of user solution options, to facilitate convergence of large, coupled problems. These options include centered or backward spatial differencing, coupled with either central or backward temporal differencing. The matrix equations may be solved iteratively (two-line successive-over-relaxation) or directly (special matrix banding and Gaussian elimination). 
TELEPHONE: $(510)$ 527-9876

FAX: (510) 527-8164

E-MAIL: MW@GEOTHERMEX.COM

\subsubsection{Elastic/Mechanical Behavior}

SWIFT98 does not consider rock deformation.

\subsubsection{Typical Application}

The original version of SWIFT98 was used by in the 1980s for the US DOE Crystalline Repository Project and later in DOE's Basalt Waste-Isolation Project and Salt Repository Projects. In these projects, SWIFT98 was used to set up site-scale (1 to $10 \mathrm{~km})$ models of the potential repository sites. These models were calibrated to field hydraulic test data, surface water measurements, infiltration data, and hydraulic pressure measurements. The models were then used to model repository operation, studying for example the effects of brine migration for repositories in bedded salt, and the effect of thermal convection due to repository-generated heat on radionuclide transport and regional groundwater flow.

\subsubsection{Applicability to EGS Simulation}

SWIFT98 was designed to provide coupled heat and mass flow and transport modeling for radioactive-waste-disposal applications, and would be applicable to a wide range of heat- and mass-flow and solute-transport modeling applications for EGS, without requiring any significant modifications or improvements.

SWIFT98 uses a finite-difference formulation, which provides rapid and reliable convergence over a broad range of conditions. SWIFT98 can be used to provide first-order approximate coupled heat- and mass-flow solutions for EGS using simple one-dimensional models for more efficient solution. For more complex cases, SWIFT98 can be run with heterogeneous material properties in two and three dimensions. SWIFT98 models can be nested to provide detailed modeling at the wellbore scale within regional EGS simulations. 
TELEPHONE: (510) 527-9876

FAX: $(510) 527-8164$

E-MAIL: MW@GEOTHERMEX.COM

SWIFT98 does not provide any coupling to stress-deformation features. The use of the finitedifference approach limits it to orthogonal geometries, making SWIFT98 unsuitable for interface with DFN approaches or for modeling complex geologic structures.

\section{$5.11 \underline{\mathrm{UDEC}}$}

\subsubsection{Overview}

UDEC (Universal Distinct-Element Code) developed and distributed by Itasca Consulting Group. UDEC is a two-dimensional distinct-element code for coupled thermo-mechanical and hydro-mechanical analyses. UDEC's capabilities include:

- simulation of large displacements (slip and opening) along distinct surfaces in a discontinuous medium;

- modeling of discontinuous medium using an assemblage of discrete (convex or concave) polygonal or polyhedral blocks;

- the treatment of discontinuities as boundary conditions between blocks;

- modeling of the relative motion along discontinuities governed by linear and non-linear force-displacement relations for movement in both the normal and shear directions;

- an explicit solution scheme, giving a stable solution to unstable physical processes;

- the ability to use a mixture of rigid and deformable blocks;

- a library of material models for deformable blocks and discontinuities;

- the use of null blocks for excavation and non-linear material models for backfill simulation; 
- full dynamic capability with absorbing boundaries and wave input;

- boundary-element coupling and automatic, radially graded mesh generation for infinite domain problems;

- structural elements (including non-linear cables), with general coupling to continuum blocks or discontinuities;

- a tunnel generator;

- a statistically based joint-set generator;

- transient heat conduction and development of thermally inducted stresses and displacements;

- monitoring of model components or stored/dissipated energy;

- the ability to associate joint material models and properties with individual contacts;

- fully coupled fluid flow in joints;

- the ability to create structural elements in an inelastic material model (e.g., fiberreinforced shotcrete); and

- the ability to model tensile failure in several materials.

\subsubsection{Hydraulic, Thermal and Elastic/Mechanical Behavior}

UDEC is a two-dimensional distinct-element code for coupled thermo-mechanical analysis for discrete-block systems and coupled hydro-mechanical analysis through discontinuities. The rock masses are assumed to be an assemblage of discrete blocks (rigid or deformable) interfaced by discontinuities. For deformable blocks, an internal discretization with constant-strain triangle 


\section{GeothermEx, Inc.}

TELEPHONE: (510) 527-9876

FAX: (510) 527-8164

E-MAIL: MW@GEOTHERMEX.COM

zones (finite-difference elements) is used for slid-block deformation. The equations of motion are the governing equations and are solved by a central-difference time-march scheme. Fluid flow is conducted through discontinuities only. No poroelasticity is considered for the solid matrix. The calculation cycles for mechanical analysis is shown in the figure below.

\section{Equations of Motion of Blocks}

For rigid blocks, the equations of motion are given by:

$$
\begin{aligned}
& \frac{\partial^{2} u_{i}}{\partial t^{2}}=\frac{\sum F_{i}^{c}}{m},(\text { translation) } \\
& \frac{\partial^{2} \Theta}{\partial t^{2}}=\frac{\sum\left[e_{i j} x_{i} \sum F_{j}^{c}\right]}{I}, \text { (rotation) }
\end{aligned}
$$

where $u_{i}$ is the translational displacement vector, $F_{j}^{c}$ is the contact-force vector, $m$ is the block mass, $I$ is the moment of inertia, $\Theta$ is the angular displacement, $e_{i j}$ is the permutation tensor, $x_{i}$ is the position vector, and $\mathrm{t}$ is time.

Discontinuities are defined by contacts between blocks. Two vertex-to-edge contacts define an edge-to-edge contact representing a discontinuity, as shown in the following figure. The mechanical behavior of discontinuities is prescribed by different constitutive laws. The dilatancy of the discontinuity is assumed to be irrecoverable and limited by a maximum contact aperture. Different constitutive laws can also be prescribed for the triangle zones of solid matrix, e.g., linear elasticity or Mohr-Coulomb plasticity. 
TELEPHONE: (510) 527-9876

FAX: (510) 527-8164

E-MAIL: MW@GEOTHERMEX.COM

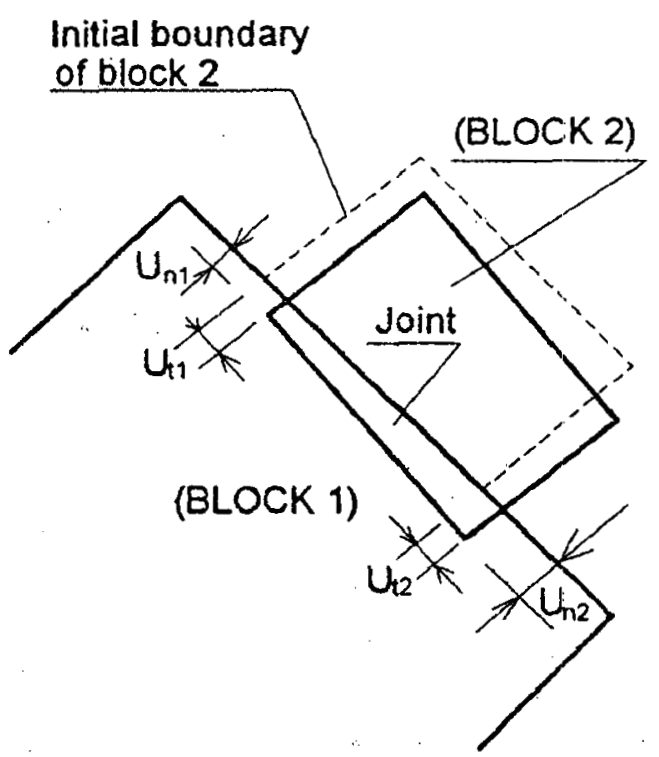

a)

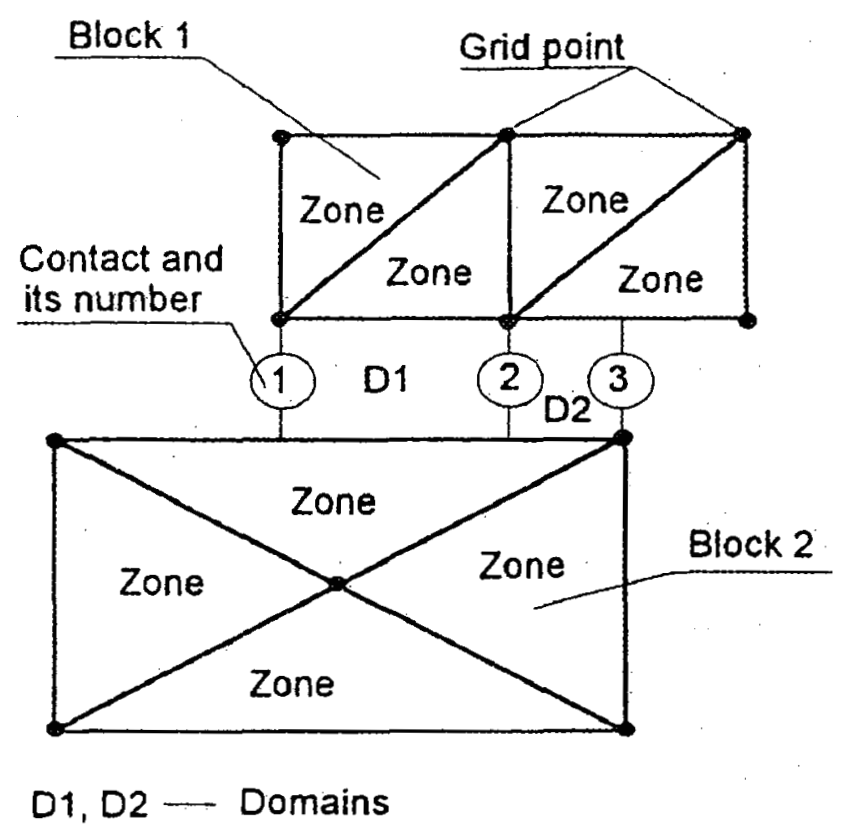

b)

a) Definition of a discontinuity in UDEC by two vertex-to-edge contacts

b) Definition of domains in UDEC for flow analysis

\section{Flow Analysis and Hydro-Mechanical Coupling}

UDEC can perform fully coupled hydro-mechanical analysis for jointed rock masses in which the conductivity of discontinuities depends on the mechanical deformation of the solid rocks and, conversely, the deformation of the solid rocks are affected by the water pressures in the discontinuities. The apertures of the discontinuities and the water pressure are updated at each time step.

Flow analysis in UDEC is performed through domains. Domains are considered to be fluid volumes that fluctuate as a function of contact normal displacement at the two ends of the 
domain. Each contact is assigned a conducting (hydraulic) aperture (a), which is related to the normal displacement $\left(\mathrm{u}_{\mathrm{n}}\right)$, by:

$$
a=e^{0}+u_{n}
$$

where $\mathrm{e}^{0}$ is the aperture at zero normal stress. A minimum (residual) aperture, $\mathrm{e}_{\mathrm{res}}$, is assumed for discontinuities to allow for some fluid conductivity under very high normal stresses, as shown by some experimental observations.

Snow's parallel-plate model is adopted for the conduction of fluid through discontinuities with the flow rate, q, defined by:

$$
q=\frac{e^{3}(\Delta P / L)}{12 \mu}
$$

where $\mu$ is the dynamic viscosity of the fluid, $L$ is the length assigned to the contact, and $\Delta P$ is the pressure difference cross a contact between adjacent domains. The fluid pressure in the domain is given by:

$$
P=P^{0}+K_{f} Q\left(\frac{\Delta t}{V}\right)-K_{f}\left(\frac{\Delta V}{V_{m}}\right)
$$

where $\mathrm{P}^{0}$ is the domain pressure at previous time step, $\mathrm{Q}$ is the sum of flow rate into the domain from all surrounding contacts, $K_{f}$ is the bulk modulus of the fluid, $\Delta t$ is the time step, and $V^{0}$ and $\mathrm{V}$ are domain volumes at previous and current time step, respectively.

The domain pressures are resolved into forces exerted by fluids at contacts and are added to the mechanical contact forces and external loads for kinematic calculation of blocks at the next time step. Therefore, total stresses are the results inside impermeable blocks, and effective stresses are obtained for contacts between blocks. 


\section{GeothermEx, Inc.}

Heat Transfer and Thermo-Mechanical Coupling

Fourier's law is used in UDEC for conductive transfer of heat within the medium with the provision for temperature, heat-flux, and convective or radiative thermal boundaries. The basic equations are written as:

$$
q_{x}=-k \frac{\partial T}{\partial x}, q_{y}=-k \frac{\partial T}{\partial y}
$$

where $\mathrm{q}_{\mathrm{x}}$ and $\mathrm{q}_{\mathrm{y}}$ are the heat flux in $\mathrm{x}$ and $\mathrm{y}$-directions, respectively, and $\mathrm{k}_{\mathrm{x}}$ and $\mathrm{k}_{\mathrm{y}}$ are the respective thermal conductivities in $\mathrm{x}$ and $\mathrm{y}$-directions. The temperature change is given by the standard heat-diffusion equation:

$$
\frac{\partial T}{\partial t}=\frac{1}{c_{p} \rho}\left[\frac{\partial}{\partial x}\left(k_{x} \frac{\partial T}{\partial x}\right)+\frac{\partial}{\partial y}\left(k_{y} \frac{\partial T}{\partial y}\right)\right]
$$

The stress change due to temperature gradient for fully deformable blocks are given by:

$$
\Delta \sigma_{i j}=-{ }_{i j} K \beta \Delta T
$$

where $\mathrm{K}$, is the bulk modulus of the solid matrix, $\beta$ is the volumetric thermal expansion coefficient of the solid, and $\Delta \mathrm{T}$ is the temperature change.

\subsubsection{Typical Application}

UDEC is typically used to model deformation in rock masses for cases in which deformations are larger than can be reasonably represented using elastic assumptions. These cases include tunnels, rock slopes, and the immediate vicinity of wellbores. Typical UDEC applications include modeling of the movement of near-wellbore fractures and resulting changes in the flow field due to oil production, and tunnel inflow calculations including the effect of rock-block movement and opening and slip of fractures. 
TELEPHONE: (510) 527-9876

FAX: $(510) 527-8164$

E-MAIL:MW@GEOTHERMEX.COM

For the Yucca Mountain project and other nuclear waste repositories in Sweden, Finland, France, and Japan, UDEC has been used to study the flow field local to canister-placement boreholes including coupled hydro-mechanical effects. UDEC models have been set up at the 10-m scale for modeling the location of individual waste canisters, and at scale of a few hundreds of meters to model fracture slip and resulting changes in flow fields due to repository construction.

\subsubsection{Applicability to EGS Simulation}

UDEC provides a truly coupled approach for simulation of flow and deformation in fractured and heterogeneous rock masses. Because UDEC uses the distinct-element approach, it directly models the mechanics of block motion in response to the water pressure and stress changes that might accompany EGS development. UDEC can therefore be used for modeling subsidence and deformations that could effect wellbore stability.

UDEC makes assumptions regarding the relationship between fracture aperture and transmissivity that have not always been validated in the field. Therefore, it is important for EGS applications to implement appropriate relationships between aperture and transmissivity. Further development of UDEC's modeling of thermal stress effects would make UDEC more directly applicable to certain EGS problems. 
TELEPHONE: (510) 527-9876

FAX: (510) 527-8164

E-MAIL:MW@GEOTHERMEX.COM

\section{OTHER SIMULATORS WITHOUT FRACTURE FLOW REPRESENTATION}

\subsection{Introduction}

Here we briefly describe other simulators that could be of use, but that presently do not include capabilities for flow in fractures.

\subsection{General Purpose Finite-Element Programs}

There are several general-purpose finite-element programs that solve a broad range of problems. They do not include the capability for flow in fractures, but include structural, heat transfer, and some fluid flow capabilities. The codes include:

- ADINA

- ANSYS/Multiphysics

- NASTRAN

- ABAQUS

- CENTRIC

- MARC

While extremely powerful, such programs do not focus on either flow in fractures or the twophase capability desired in an EGS reservoir simulator.

\subsection{CASTEM 2000 and TRIO-EF}

CASTEM 2000 is a finite-element code developed by the Technology Department of CEA (Commissariat Français à l'Energie Atomique, the French Atomic Energy Agency). CASTEM 2000 is designed for coupled thermal and mechanical analysis. CASTEM does not itself provide 
TELEPHONE: (510) 527-9876

FAX: (510) 527-8164

E-MAIL:MW@GEOTHERMEX.COM

capabilities for flow modeling, and is therefore used in an iterative manner with another CEA finite-element code called TRIO-EF for hydraulic calculations. Together, they provide capabilities for modeling of coupled thermal, hydraulic and mechanical (T-H-M) behaviors, including heat flow, mass flow and rock deformation. The two codes are loosely coupled, requiring iteration between them for solution of T-H-M problems.

TRIO-EF solves for flow by the finite-element method for single-phase flow. The coupled hydro-mechanical process is simulated in CASTEM 2000 using the concept that the porosity of the rock mass depends on plastic deformation, indicated by changes of fracture apertures.

CASTEM 2000 solves heat flow as a combination of convective and conductive heat transfer. However, since CASTEM 2000 does not include fluid flow, it is generally used primarily for conductive heat flow. When convective heat transport dominates, heat transport is modeled using TRIO-EF, which has the capability to model advective fluid flow together with convective heat transport.

To facilitate modeling of plastic as well as elastic strains, CASTEM 2000 uses the principle of virtual work for stress-strain calculations. CASTEM 2000 assumes equivalent continuum properties for the rock matrix, but provides for modeling of the effect of discontinuities within the matrix by definition of elasto-plastic material properties.

The French Nuclear Agency (NEA) typically uses CASTEM 2000 for analysis of engineered mechanics systems such as generator components, pipe system, waste overpacks, and structural supports. The use of CASTEM 2000 together with TRIO-EF for T-H-M modeling of rock mass response to canister heat production is relatively novel, but is more typical of the kinds of applications which could be expected for EGS development. CASTEM 2000 was used with TRIO-EF to model thermo-mechanical response of a fractured rock mass to a single cylindrical heater as part of the DECOVALEX project. In this experiment, CEA demonstrated that CASTEM 2000 and TRIO-EF can be adapted to work together to model T-H-M response. 
TELEPHONE: (510) 527-9876

FAX: (510) 527-8164

E-MAIL:MW@GEOTHERMEX.COM

CASTEM 2000 is designed to allow the user to specify complex, non-linear mechanical properties using elasto-plastic material properties. TRIO-EF is designed to facilitate porousmedium flow modeling with complex, 3-D geometries. Both of these features are potentially useful for EGS simulation of both the engineered and natural geological components of the system. However, CASTEM is designed for sophisticated users who wish to customize the models' constitutive laws, rather than to quickly and efficiently solve engineering problems.

TRIO-EF is limited to porous-medium flow solutions, and is therefore not applicable to many fractured reservoirs. CASTEM's use of equivalent elasto-plastic constitutive relationships rather than discrete modeling of the mechanics of rock joints requires significant care in application.

\subsection{CFEST}

CFEST (Coupled Fluid, Energy and Solute Transport) is a simulation code developed Battelle Pacific Northwest Laboratory (BPNL) for the US DOE. It is available for public distribution in its original form through the DOE National Engineering Software Center. It is also available in commercial versions with a relatively modern user interface. CFEST was developed by BPNL for the study of multi-layered, non-isothermal groundwater systems. It can model discontinuous as well as continuous layers, time-dependent and constant source/sinks, and transient as well as steady-state flow. The finite-element method is used for analyzing isothermal and nonisothermal events in a confined-aquifer system. Only single-phase Darcian flow is considered. CFEST does not consider rock deformation.

For the last two decades, the CFEST code has been used by DOE, EPA and DOD to evaluate to several public-agency and industrial sites, including several large, complex groundwater basins and Superfund sites.

Unlike most hydrogeological simulation platforms, CFEST directly combines coupled heat- and mass-flow modeling with solute-transport simulation capabilities. As a result, CFEST provides a powerful EGS platform for situations in which tracer tests can be used to calibrate and condition 
the same model used for heat and mass flow modeling. CFEST is particularly useful in cases where large thermal gradients induce flows that cannot be properly simulated in conventional flow and transport models.

\subsection{FEMWATER}

FEMWATER, developed for the US Environmental Protection Agency (EPA) by Prof. G. Yeh (currently at Pennsylvania State University) is a three-dimensional finite-element-based groundwater-flow code. FEMWATER is available for free download from the a number of web sites, and is also available commercially as part of the US Department of Defense (DOD) GMS groundwater modeling systems.

FEMWATER calculates water velocity, moisture content, and pressure head in unsaturated and saturated porous media. FEMWATER is available in two- and three-dimensional versions, and also has companion solute-transport codes FEMWASTE (2-D) and 3DLEWASTE (3-D).

Because FEMWATER is distributed as open source code, a variety of custom versions have been developed. FEMWATER could be directly modified to include, for example, effects of heat transport or deformation.

FEMWATER and 3DFEMWATER perform two- and three- dimensional time-dependent fluid flow analyses using the finite-element method. FEMWATER's particular strength is in its treatment of moving phreatic surfaces and water flow in unsaturated vadose zones using a generalized Richard's equation. FEMWATER is a good choice for modeling complex threedimensional geometries, because it is able to combine hexahedral, tetrahedral, and triangular prism elements. FEMWATER/3DFEMWATER are single-porosity porous-medium codes, and do not include discrete-fracture network (DFN) modeling capabilities.

Heat transport is not considered in FEMWATER. However, Boss International has developed a customized FEMWATER companion, 3DFEMFAT. 3DFEMFAT is a commercial code which provides the capability to model thermal conduction and convection using the same 
TELEPHONE: $(510)$ 527-9876

FAX: (510) 527-8164

E-MAIL: MW@GEOTHERMEX.COM

FEMWATER finite-element formulation and geometry. FEMWATER does not model rock deformation.

FEMWATER is an uncoupled three-dimensional groundwater flow model. Its primary usefulness arises from its integration with the US Department of Defense GMS Package, which provides a modeling environment that combines model development and post-processing with basic GIS and CAD capabilities. FEMWATER/GMS could be useful for EGS in cases where there is a need to rapidly prototype a flow model for a complex geology that has been previously defined using a GIS system such as EarthVision or Arc/Info. FEMWATER has historically had significant problems with numerical stability, but these have reportedly been solved.

\subsection{NAMMU}

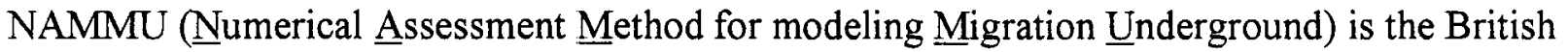
company AEA Technology's finite-element software package for modeling groundwater flow and transport in porous media. It is available for commercial licensing. NAMMU incorporates three-dimensional grid-generation and post-processing capabilities. One advantage of NAMMU is that it allows the user to nest meshes of different scales to obtain greater detail in regions of interest.

NAMMU couples heat and mass flow, and can be integrated with the NAPSAC discrete-fracture network (DFN) code to provide a mixed DFN/porous-medium modeling environment.

NAMMU uses the finite-element method to solve coupled mass and heat flow and solute transport in three dimensions. NAMMU models single-phase, single-porosity flow under both saturated and unsaturated conditions. Unsaturated groundwater flow is modeled using a characteristic-curve approach.

NAMMU includes the ability to model anisotropic hydrologic properties. In addition to threedimensional modeling using volume elements, NAMMU can carry out two-dimensional 
E-MAIL:MW@GEOTHERMEX.COM

simulation of both cross-section and areal flow. NAMMU's solute-transport algorithm has the ability to model multiple, decaying tracers with advection, dispersion, and sorption.

NAMMU solves heat, solute transport, and mass flow in a single finite-element formulation, which facilitates coupled modeling. Fluid and material properties can be defined as direct functions of temperature, pressure, and solute concentration.

NAMMU models thermal conduction using the finite-element method. It does not model thermal convection or radiation. However, because it solves the combined heat flow/mass flow equation, no iteration is required between heat flow and mass flow. NAMMU does not consider rock deformation.

NAMMU is used primarily by its owner and developer, AEA Technologies, although it is also available commercially. NAMMU's characteristic-curve approach for modeling the unsaturated zone makes it well suited for regional modeling, which is NAMMU's typical application. This is also facilitated by NAMMU's solute-concentration-dependent fluid-density capabilities, which allow NAMMU to model salt/fresh water interfaces and density dependent flows.

NAMMU has been used to develop regional-scale (1- to 10-kilometer) models for sites including Sellafield, UK; Äspö, Sweden; and Gorleben and Morsleben, Germany.

NAMMU's strength for EGS applications lies in its ability to model to high levels of detail within very large-scale three-dimensional regional hydrogeologic models by using model nesting. NAMMU solves coupled density-dependent flow and solute transport, which is potentially useful for EGS, in cases where density-dependent flows might be significant due to both thermal and dissolved-solute effects. .

NAMMU is somewhat complex to run, and lacks the level of integration with GIS and CAD systems of more commercially orientated systems. However, for experienced users, NAMMU provides a powerful platform for regional scale modeling. 


\subsection{PHOENICS}

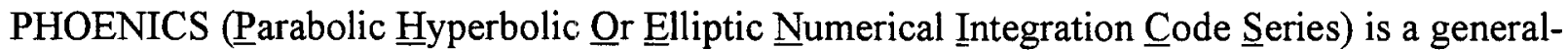
purpose computational fluid-dynamics code developed and distributed by Concentration, Heat \& Momentum Ltd. (CHAM). Shareware as well as commercial versions of PHOENICS are available from CHAM.

PHOENICS was designed as a general-purpose solver, using an integrated finite-difference method to solve a broad spectrum of computational fluid dynamics problems. As a generalpurpose code, PHOENICS is used for a very wide range of flow problems including heating/ventilation, turbine engines, airplane design, chemical engineering, and oil-spill modeling.

Since PHOENICS is a general-purpose code rather than a groundwater-flow or heat-flow simulator, it requires considerable expertise in computational fluid dynamics (CFD) for practical applications. PHOENICS includes a powerful simulation language as well as customized modules to allow simulation of any desired combination of process, and any 1-D, 2-D, or 3-D geometry. For EGS, PHOENICS can be used to model all of the relevant processes of heat and mass transport including advection, convection, and conduction both in the engineered and natural systems. PHOENICS can also be used to model effects of deformation and processes of precipitation and chemical reaction and the attendant effects of flow.

As a general-purpose solver, PHOENICS is able to solve a much broader range of problems than other codes. Processes of concern to EGS geothermal development include:

- turbulent as well as laminar flow;

- solute transport with chemical reactions;

- multi-phase/multi-component flow; 


\section{GeothermEx, Inc.}

TELEPHONE: (510) 527-9876

FAX: (510) 527-8164

E-MAIL:MW@GEOTHERMEX.COM

- chemical reactions such as precipitation and dissolution, and their effect on flow;

- convective, advective, conductive, and radiative heat transport; and

- stress-strain effects.

PHOENICS can solve these in one, two or three dimensions. As a result, PHOENICS can solve the physics of flow in both engineered and natural components of EGS systems. Unique features of PHOENICS include an ability to solve for multi-fluid turbulence, simultaneous solution of stress/strain and flow problems, and modeling of interspersed solids and fluids.

Since PHOENICS is customizable, models can be implemented as single-porosity, dual-porosity, or dual-permeability. A module has been developed to allow PHOENICS to solve combined porous-medium and discrete-fracture network (DFN) code.

PHOENICS can solve stress-strain for any combination of user-specified mechanical properties and boundary conditions, including elastic, elasto-plastic, and brittle failure models.

PHOENICS solves the physics of deformation together with the physics of heat and mass flow, and therefore these processes can be fully coupled if desired. For example, thermo-elastic stresses together with mechanical stress changes can be used to model strains and their effect on the integrity of well equipment and structures, and also their effect on fracture permeability.

Typical applications of PHOENICS are in the areas of chemical, mechanical, and nuclear engineering such as turbine design and design of chemical process equipment. PHOENICS has been used in the SKB nuclear waste isolation project in Sweden to model regional-scale (1- to $10-\mathrm{km}$ ) groundwater flow and transport, including surface-water infiltration, moving phreatic surface, and density effects due to varying groundwater salinity. PHOENICS has also been used to model, for example, the physics of oil spills, and the use of steam floods for enhanced oil recovery. 
PHOENICS is a modular, three-dimensional, coupled-process continuum code. As a result of its modular design, PHOENICS can be used to solve any combination of coupled processes, including heat flow, solute transport, mass flow, and deformation. PHOENICS could therefore be valuable for any EGS application in which coupled processes are a significant focus.

However, since the user must define the equations to be solved, PHOENICS requires an expert user for most applications. PHOENICS has been used in radioactive-waste applications primarily for modeling variable-density mass flow and conductive heat transport. PHOENICS could be improved for EGS applications by developing a custom version that solves for the range of material properties and processes of concern for EGS, without requiring user specification of equations.

\subsection{PORFLOW W}

PORFLOW W is developed and distributed commercially by the French company ACRI. PORFLOW is a comprehensive computer program for simulation of transient or steady- state flow, heat, salinity and mass transport in multi-phase, variably saturated, porous or fractured media with dynamic phase change. The geometry may be two- or three-dimensional, Cartesian or cylindrical. The porous/fractured media may be anisotropic and heterogeneous, arbitrary sources of sinks (injection or pumping wells) may be present and, chemical reactions or radioactive decay may take place. It accommodates alternate fluid- and media-property relations and boundary conditions.

The PORFLOW program is highly modular and has been applied to a wide range of practical problems in petrochemical, hydrological, geological, nuclear and chemical industry applications.

PORFLOW numerically solves a variable set of equations for general transport, multi-phase pressure, temperature and one or more chemical species. Constitutive equations, phase-change relations, equations of state, and initial and boundary conditions supplement the governing equations. The equations are coupled through convection, buoyancy, temperature, phase-change, 


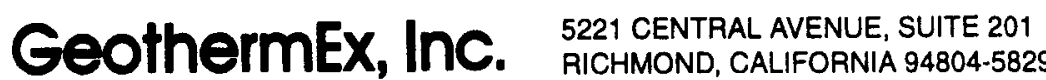

TELEPHONE: (510) 527-9876

FAX: (510) 527-8164

E-MAIL: MW GEOTHERMEX.COM

fluid-density and viscosity effects. These equations may be solved individually or simultaneously in a coupled or uncoupled manner, depending on the needs of a specific problem and the options selected by the user.

The current version of PORFLOW permits simulation of flow systems with up to three phases. Examples of such systems are water-oil-vapor-air, water-steam-air, water-steam, water-ice or water-air systems.

The method of Nodal Point Integration (NPI) is employed for integration of the governing differential equations by temporal and spatial discretization over each volume (element) of the physical domain. It leads to solutions that automatically conserve fluid, heat, and mass locally within every grid element, as well as for the entire flow domain. The storage terms are approximated by a modified Newton-Raphson method. The dependent variable or its change from the current state approximates the flux terms. The elements used to define the problem geometry can vary in size, but their shape is restricted to that of a quadrilateral, hexahedral or segment of a cylinder.

The resulting matrix of algebraic equations can be solved be one or more of several matrix inversion algorithms. The available options include the Point Successive Over-Relaxation, the Alternating Direction Implicit (ADI), the Conjugate Gradient, Cholesky Decomposition and Gaussian Elimination. In addition, the software provides the flexibility to use any other matrix inversion technique through coupling with an external matrix-inversion algorithm. PORFLOW does not consider rock deformation.

PORFLOW is typically applied where more complex coupled processes such as multi-phase flow with variable saturation, density-dependent flow, and flow with phase changes and chemical reactions are required. Example applications include simulation of tidal zones where fresh and salt water meet, energy storage through injection of hot water into cold-water aquifers, and flow and transport for radioactive-waste-repository performance assessment. PORFLOW 
TELEPHONE: $(510) 527-9876$

FAX: $(510)$ 527-8164

E-MAIL:MW@GEOTHERMEX.COM

can model both simple, 1-D geometries, and finely discretized, complex 3-D geological structures.

PORFLOW can be considered as a fully developed, commercial, mature simulator that includes many of the features desirable for EGS flow simulation. PORFLOW models flow of liquid and steam phases, with dynamic phase changes, and directly couples solute transport and density effects. PORFLOW can be used to develop both two-and three-dimensional models, with anisotropy and heterogeneous material properties. It might even be possible to use PORFLOW to model mineral precipitation and its dynamic effect on fracture transmissivity. PORFLOW models variable-density flow. PORFLOW does not directly consider fracture geometry, but can be adapted to work with a DFN generator to provide this capability. PORFLOW also does not directly couple stress/deformation. However, its modular structure makes it possible to include this feature if required.

\subsection{SUTRA}

SUTRA was developed and distributed by the US Geological Survey as shareware. SUTRA uses a hybrid integrated finite-difference/finite-element algorithm to solve saturated-unsaturated, fluid-density-dependent groundwater flow with heat transport or chemically reactive singlespecies solute transport. SUTRA is a two-dimensional code, and may be used for areal and cross-sectional modeling of saturated ground-water flow systems, and for cross-sectional modeling of unsaturated zone flow. As a shareware code, SUTRA is widely distributed, and can be considered an industry standard for modeling of coupled heat/mass flow and density dependent, saline interface modeling.

SUTRA is a single-phase, single-porosity porous-medium continuum flow and transport code. SUTRA solves groundwater flow, heat transport, and solute transport using the same integrated finite-difference/finite-element solver. The finite-element/integrated finite-difference approach 
TELEPHONE: $(510)$ 527-9876

FAX: (510) 527-8164

E-MAIL:MW@GEOTHERMEX.COM

uses quadrilateral elements, which allows for implementation of realistic, complex heterogeneous geologies.

Fluid properties such as density and viscosity can be defined as functions of pressure and temperature. Flow properties such as permeability and storativity can be defined as functions of temperature, saturation, and orientation. SUTRA models saturated and unsaturated systems using a characteristic-curve approach.

The solver for SUTRA is based on a two-dimensional hybridization of finite-element and integrated finite-difference methods employed in the framework of a method of weighted residuals. The time discretization used in SUTRA is based on a backward finite-difference approximation for the time derivatives in the balance equations. The SUTRA solver requires careful definition of the spatial and temporal discretization to obtain stable and reliable convergence. SUTRA does not consider rock deformation.

SUTRA is typically used where coupled flow properties are important, due to either high concentrations or thermal gradients. SUTRA has been used to model groundwater flow in the unsaturated zone for the Yucca Mountain project, and the effect of saline concentrations on large scale $(10 \mathrm{~km})$ flow for the Sellafield, UK radioactive waste project. SUTRA has also been used to model groundwater flow with solute transport for contaminated sites, and has been used extensively in modeling variable-density leachate movement, fresh/salt water interfaces, and saltwater intrusion in aquifers in near-well or regional scales

SUTRA heat transport simulation has been used to model thermal regimes in aquifers, subsurface heat conduction, aquifer thermal-energy-storage systems, geothermal reservoirs, thermal pollution of aquifers, and natural hydrogeologic convection systems.

SUTRA is a leading code for two-dimensional modeling of heat and mass flow with variable density and saturation. As a result, SUTRA has been used for coupled heat/mass flow simulation of geothermal reservoirs worldwide, including, for example, The Geysers in California. 


\section{GeothermEx, Inc.}

SUTRA can be used in EGS applications to provide initial values for locations of vapor/fluid front movement, or for heat- and mass-flow calculations. SUTRA's solver is poorly conditioned, such that the code does not converge for a wide range of problems. Therefore, the most important improvement to SUTRA for EGS applications would be improvements to SUTRA's solver technology. With these improvements, and improved user interfaces, SUTRA could be a valuable tool for wellbore- and reservoir-scale EGS simulation.

\subsection{THAMES}

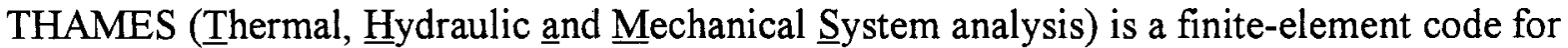
fully coupled T-H-M processes in saturated or unsaturated geological media, developed for and used by JNC (Japanese Nuclear Fuel Cycle Development Institute). JNC is responsible for highlevel radioactive-waste-repository research and feasibility assessment for Japan, and operates underground research labs for the study of flow and transport in fractured rock.

THAMES is a continuum (porous-medium) code, but incorporates the effects of fractures using effective-permeability tensors derived from fracture geometries by using the concept of crack tensor. The fully coupled T-H-M processes considered in THAMES are based on the following assumptions:

- the medium is porous and elastic;

- Darcy's Law is adopted for water flow in saturated and unsaturated media;

- Fourier's Law is adopted for heat transfer in both solid and liquid phases when no consideration given for gas phase;

- there is no consideration of phase change between water and vapor; and

- the density of water changes with both temperature and pressure. 
TELEPHONE: (510) 527-9876

FAX: (510) 527-8164

E-MAIL: MW@GEOTHERMEX.COM

THAMES was used by KPH (a collaboration of Kyoto University, JNC and Hazama Corporation) during the DECOVALEX project to simulate coupled heat and fluid flow and fracture deformation in heated rock block experiments.

THAMES provides basic coupled processes for a single-porosity, anisotropic continuum. However, it does not provide special features such as aperture coupling, phase transitions and convective heat transport, which are particularly useful for EGS applications. In addition, THAMES distribution status is unclear.

\subsection{TRACR3D}

TRACR3D was developed for the US DOE through Oak Ridge National Laboratory, and it is now distributed commercially through organizations including GeoComp. TRACR3D models three-dimensional, time-dependent, multi-phase, multi-component, reactive flow through porous and fractured media. Capabilities also include the ability to accurately represent complex threedimensional geologic media and structures and their effects on subsurface flow and transport.

TRACR3D can simulate the flow of air and water in saturated and unsaturated media. It can handle multiple chemically reactive, radioactive and sorbing tracers.

TRACR3D is a single-porosity, porous-medium model, optimized for solving multi-species transport in saturated/unsaturated porous media. TRACR3D using a finite-difference algorithm to solve coupled flow and solute-transport equations. TRACR3D's flow model can handle 1-D, 2-D, and 3-D calculations.

Transport mechanisms solved by TRACR3D include advection, diffusion, dispersion, volatilization, radioactive decay and decay chains, and sorption. TRACR3D does not consider heat transfer. TRACR3D does not consider rock deformation.

TRACR3D is typically used for simulation of solute transport in saturated/unsaturated geologic settings. For example TRACR3D has been used to model the saturated/unsaturated interface at 
Yucca Mountain, and for assessment of low-level radioactive waste transport at the site (a few hundreds of meters) scale. TRACR3D is used to simulate solute transport with volatilization for environmental assessment, and it has been adapted for simulation of bio-remediation.

TRACR3D is a three-dimensional tracer-transport code that can be used in EGS applications that require simple modeling of tracer tests, without consideration of coupled processes. TRACR3D can be improved for EGS applications by combining it with other coupled heat/mass flow codes to provide solute-transport features for codes that lack such capabilities. 
TELEPHONE: (510) 527-9876

FAX: (510) 527-8164

E-MAIL:MW@GEOTHERMEX.COM

\section{OBSERVATIONS AND DISCUSSION}

\subsection{Categorization}

In attempting to group, categorize, and evaluate the codes described in the previous sections, it is useful to focus on the approaches used to represent flow in fractures. Four broad approaches can be identified:

- Discrete representation of the fractures and rock matrix, as used in FRACTure and Geocrack2D. This approach attempts to directly model each significant fracture and to directly model the rock matrix, which assumes detailed knowledge of the reservoir. The advantage is that the model should provide realistic simulations with fewer approximations. The challenges are to develop methods to easily create such models and then to obtain solutions. In addition, the conceptual model (on which the numerical model is based) must be detailed enough to specify which fractures control fluid flow.

- Focus on the rock matrix, using approximations for fracture flow, as used in GEOTH3D and all of the hydrothermal simulators. Blocks of fractured rock are modeled by specifying fracture spacing and the hydraulic properties of the matrix and the fractures in a dual porosity/dual permeability approach, thus yielding effective hydraulic properties for the block. The advantage is that such an approximation allows relatively simple representation of a reservoir, providing rapid solutions in situations where the rock matrix is highly fractured or porous. Information about specific fractures is not required. However, some detail is lost in making the porous flow approximation. For example, the sharp temperature gradients in hot rock on either side of a fracture filled with cool water are difficult to represent, and short-circuiting of injected water back to the production well along an appropriately situated fracture could be missed, Therefore, the use of porous-media simulators can lead to more optimistic reservoir predictions than warranted, although the MINC method of TOUGH2 circumvents some of these problems. 
- Using a porous-medium simulator to model large-scale, discrete fractures. Geothermal systems with such features can be represented in a numerical model using an appropriately arranged set of long and narrow gridblocks with very high porosity and permeability. This approach has proven satisfactory for several hydrothermal projects.

- Focus on the fractures, using approximations for the matrix, as used in FRACSIM-3D and FracMan. These simulators typically use a stochastic approach to develop a fracture network model, sometimes with thousands of fractures. The fractures provide the connections in the global model, and each fracture is associated with a local rock matrix (similar to the dualporosity approach). The advantage is that complex fracture networks can be modeled, the disadvantage is the approximation to the matrix.

\subsection{Current Capabilities Relative to Desired EGS Features}

In the following sections, the current capabilities of the simulators reviewed are compared with the necessary and desired features of an EGS simulator identified in Section 2. Tables $1-3$ summarize the features of all simulators reviewed.

\subsubsection{Explicit Representation of Fractures}

All simulators (except those in Section 6) can be used to model fractures at some level. As discussed above, the mathematical formulation that describes the fractures and the ease with which fractures can be represented differ from one simulator to the next. Two of the HDR simulators (FRACTure and Geocrack2D) can represent fractures discretely; FRACSIM-3D does so in simulating hydraulic fracturing operations only (Table 1). Several of the nuclear-wasteisolation codes (FracMan, HYDREF, MAGNUM2D, MOTIF, PHOENICS, ROCMAS and UDEC) allow discrete fractures to be represented (Table 3).

Like all four hydrothermal codes (Table 2), GEOTH3D uses a porous medium approach; FRACSIM-3D also uses this method to simulate normal production and injection (as opposed to 
TELEPHONE: (510) 527-9876

FAX: (510) 527-8164

E-MAIL: MW@GEOTHERMEX.COM

stimulation). As mentioned above, all of the porous-medium simulators allow approximate representation of large-scale discrete fractures using long and narrow gridblocks with high porosity and permeability.

\subsubsection{Fracture Opening as a Function of Effective Stress}

Three of the four HDR simulators (Table 1) and many of the nuclear-waste-isolation simulators (Table 3) include approximations of this, either through permeabilities that are a function of stress or by discrete-fracture modeling. None of the hydrothermal simulators (Table 2) have this feature.

\subsubsection{Shear Deformation and Associated Jacking of Fractures}

Of the HDR simulators, FRACTure and FRACSIM-3D include this feature (Table 1), which is particularly important as fractures grow during stimulation operations. As in the case of fracture opening in response to changes in fluid pressure, none of the hydrothermal simulators can model this, nor can any of the nuclear-waste-isolation simulators.

\subsubsection{Relationship Between Fracture Aperture and Conductivity}

Three of the four HDR simulators (Table 1) and several of the nuclear-waste-isolation simulators (Table 3) use the cubic law to define the relationship between fracture aperture and conductivity.

\subsubsection{Channeling in Fractures}

Only one HDR simulator (FRACTure) handles this feature, and does so approximately by manually adjusting fracture element properties. None of the hydrothermal simulators take account of channeling. In two nuclear-waste-isolation simulators (FracMan and HYDREF), channeling is accounted for by using pipe-like elements, often located at the intersection of two fractures. 


\subsubsection{Thermo-Elastic Effects}

All of the HDR simulators except GEOTH3D include this feature (Table 1); FRACSIM-3D handles thermo-elastic effects using a global stress rather than a local elasticity solution. All of the conventional hydrothermal simulators can approximate this effect by varying bulk porosity and permeability with pressure and temperature. However, they cannot simulate, for example, the thermal contraction of impermeable rock, which changes the aperture (and therefore, possibly, the conductivity) of a fracture. Many of the nuclear-waste-isolation simulators handle thermo-elastic effects.

\subsubsection{Mineral Deposition and Dissolution}

Only one of the HDR simulators (FRACSIM-3D) includes a simple mineral deposition and dissolution. One nuclear-waste-isolation simulator (PORFLOW W) has this capacity also.

\subsubsection{Tracer Module}

All simulators reviewed here provide tracer modules.

\subsubsection{Multi-Phase Flow}

All the conventional hydrothermal simulators and a few of the nuclear-waste-isolation simulators provide multi-phase flow capability. None of the HDR simulators have this ability.

\subsection{Implementation of Features}

Discrete fractures can, in some form, be implemented in most of the simulators reviewed. In porous-medium flow models discrete fractures can be modeled by defining the hydraulic features of sets of thin, adjacent grid blocks. Although this approach has been used to model systems in which flow is dominated by a few, major fractures, it is cumbersome to implement in systems with many fractures. At least one fracture mesh generator (Golder Associates' FracMan) has 
TELEPHONE: (510) 527-9876

FAX: (510) 527-8164

E-MAIL: MW@GEOTHERMEX.COM

been adapted to two of the hydrothermal codes (TOUGH2 and FEHM), enabling them to represent fractures explicitly as a series of 2-D, triangular elements. This has not been used extensively as far as we know, but holds promise for easing the development of hydrothermal models with many discrete fractures.

Changing fracture apertures with effective stress enables a more accurate representation of reservoirs with low natural permeability or when permeability enhancements are being modeled. Mathematical models describing this phenomenon have been implemented in the fracture-based simulators. None of the hydrothermal (porous-medium) codes incorporate deformation of the rock matrix, which is needed to calculate aperture changes. A significant effort would be required to implement deformation in these codes, and it may not be possible in all codes.

Changing fracture conductivity with aperture is implemented in discrete-fracture simulators using the cubic law:

$$
Q=-\frac{a^{3}}{12 \mu} \rho \Delta P
$$

where $Q$ is the flow rate, a is the fracture aperture, $\mu$ is the dynamic viscosity, $\rho$ is the fluid density, and $\Delta P$ is the pressure drop. The cubic law is a reasonable approximation of what happens when a fracture is inflated; however, it cannot be used for two-phase flow.

In multi-phase porous-flow models, ignoring capillary pressure, fluid flow in a fracture can be expressed as:

$$
\begin{aligned}
& Q_{w}=-A k \frac{k_{r w}}{\mu_{w}} \rho_{w} \Delta P \quad \text { and } \\
& Q_{s}=-A k \frac{k_{r s}}{\mu_{s}} \rho_{s} \Delta P
\end{aligned}
$$


where $A$ is the cross-sectional area normal to the direction of the fluid flow, $k$ is the absolute permeability, $k_{r}$ is the relative permeability to the phase under consideration $\left(k k_{r}\right.$ is therefore the effective permeability to the phase), and the subscripts $w$ and $s$ refer to the liquid and steam phases, respectively. However, this can only be assigned as a hydraulic property to an appropriately arranged grid block set, and would not be a dynamic feature as in the simulators that use the cubic law. In theory, it is possible (with suitable experimentation) to develop empirical relationships for differing amounts of steam and water that would allow the cubic law to be adapted for two-phase flow. However, considering the level of uncertainty that continues to be associated with the relative permeabilities of water and steam in porous media after many years of work, any such experimentation is unlikely to yield applicable results in the near future.

Flow channeling is one of the desirable features for which a mathematical model (pipe flow) exists; including pipe elements in a model can be done relatively easily. However, there are two difficulties associates with representing channeled flow in a numerical simulation. First, one must define where channeling is occurring from field data. Although certain pressure transient analysis methods can indicate channel-like (i.e., one-dimensional) flow, the location and orientation of the channel can only be inferred. Obtaining sufficient detailed knowledge to successfully identify when channeling is occurring will require input from other fracture detection and characterization technologies, which are under development but may not be useful in the near future. Second, as was mentioned previously in the description of porous-medium simulators in Section 7.1, the simulation mesh must be fine enough to capture the sharp gradients associated with flow in a small channel, and the inclusion of small, cylindrical elements with random orientations presents difficulties in regard to both designing the grid and computing the results.

For thermo-elastic effects, stress in the rock due to temperature change can be expressed as:

$$
\sigma_{\mathrm{T}}=\mathrm{CK} \beta_{\mathrm{T}} \Delta \mathrm{T}
$$


where $\sigma_{\mathrm{T}}$ is the thermal stress, $\mathrm{C}$ is a constant, $\mathrm{K}$ is the bulk modulus of the rock, $\beta_{\mathrm{T}}$ is the coefficient of thermal expansion, and $\Delta \mathrm{T}$ is the temperature change.

This stress, in addition to the stress imposed because of fluid pressure, can alter the fracture aperture, which changes the fluid flow in the fracture. Since aperture cannot be measured directly, it must be inferred through the transient and steady-state flow simulation and by comparison with tracer data. In simulators that handle discrete fractures, the thermo-elastic effect on fracture aperture can and has been implemented. In the conventional hydrothermal simulators, bulk porosity and permeability can vary with pressure and temperature, but impermeable blocks would not be subject to any such effects. As in the case of allowing fracture apertures to change with effective stress, deformation would have to be incorporated into the hydrothermal simulators to enable them to effectively represent the thermal contraction of hot matrix blocks (and the resulting change in fracture aperture between the matrix blocks) in response to injection of cold water.

All the reviewed simulators handle tracers fairly effectively; therefore, no further discussion is provided here.

Mineral deposition and dissolution has been implemented approximately in FRACSIM-3D with user-specified temperature-dependent reaction rate constants and saturation concentrations. The aperture (and therefore the conductivity) of the fractures changes as minerals are deposited or dissolved. Of the hydrothermal simulators, a reactive chemical transport model has been developed to work with TOUGH2. This augmented simulator (TOUGHREACT) permits a wide range of chemical processes to be modeled, including mineral deposition and dissolution. After further evaluation and testing, it will be available for use with TOUGH2 and will operate in an iteratively coupled mode. Mineral precipitation and dissolution is calculated on a gridblock-bygridblock basis; porosity and permeability are not affected. However, estimates of the effect on porosity could be made fairly easily. Implementing a full set of chemical equilibria and reaction kinetics within TOUGH2 was investigated and found to be too cumbersome for practical use. 
The difficulty encountered in trying to solve chemical reactions within a numerical model of a geothermal system suggests that a de-coupled approach would be preferable if such a feature is to be implemented. However, the lack of this feature is not a hindrance to EGS development. In fact, in more than 40 years of operating hydrothermal systems, which are much more likely to have scaling problems than HDR reservoirs owing to the higher enthalpy and fluid chemistry, scaling is an operational consideration but never a "show -stopper." The most detrimental mineral precipitation (calcite and silica scaling) occurs upon boiling in the wellbore or reservoir. There are no hydrothermal reservoirs we know of where mineral deposition has had a significant effect on permeability, except in the region immediately adjacent to the wellbore. Even at Cerro Prieto, which is the most extreme documented case where the boiling front has clearly moved into the reservoir, scale is removed from the near-wellbore region by acid jobs and other techniques. The bulk of the reservoir has not been affected.

The ability to handle two phase flow has not been necessary in evaluating HDR reservoirs to date. However, this is likely to become a limitation if HDR simulators are to be considered for evaluating EGS projects adjacent to existing hydrothermal systems with extensive two-phase conditions. New governing equations for two-phase flow could be incorporated into HDR simulators, but would require a significant effort.

\subsection{Summary}

It is apparent from the above discussion that, while each of the simulators has many of the capabilities listed above, none has all of them; each simulator has its strengths and weaknesses. Furthermore, the ease of implementing features varies with simulator type. A single type of simulator may not be suitable for all EGS projects or at every stage of a given project. For example, in the early development stage of an EGS project, when available information is limited and the primary need is for reserves estimation and project planning, porous-media or fracture-network simulators would be more practical to use. In a more mature stage of the same 
TELEPHONE: (510) 527-9876

FAX: (510) 527-8164

E-MAIL: MW@GEOTHERMEX.COM

project, when reliable information on fractures becomes available, discrete-fracture models may become preferable for optimizing the injection/production strategy.

It is our opinion that at this time, further development of existing simulators is more useful than developing a single, all-purpose simulator for EGS applications. This is particularly true considering that near-term EGS development in the US is likely to take place in hot, lowpermeability areas in or around existing hydrothermal fields. Here, a field operator will need to use numerical simulation to predict the effect of the EGS development on conditions in the main part of the reservoir. Considering that nearly all reservoirs developed for geothermal power production have two-phase conditions, a conventional hydrothermal simulator must be used for the present. Such simulators have the proven capability and robustness to handle perhaps thousands of gridblocks and hundreds of wells, and there is already a level of familiarity with their use by the field operators. Although such simulators will have to handle the fracture-related features in an approximate way and cannot represent the dynamic aspects of fractures, this should not hinder the advancement of EGS developments collocated with hydrothermal projects.

In the longer term, a stand-alone EGS project might require a dedicated EGS simulator that combines the capabilities of HDR and hydrothermal simulators, and possibly some of the features of the more complex nuclear-waste-isolation simulators. Interfaces have been developed to link Golder Associates' FracMan discrete fracture network generator with both TOUGH2 and FEHM; this enables these traditionally porous-medium simulators to rigorously model flow in discrete fracture, which is one way to approach the development of a more comprehensive set of simulation tools.

If hydraulically active fractures could be identified and characterized, then it would be appropriate to pursue including some of the fracture-related features (fracture opening as a function of effective stress, the relationship between fracture aperture and conductivity, shear deformation and channeling). As research into the identification and characterization of 
GeothermEX, InC. $\begin{aligned} & \text { 5221 CENTPAL AVENUE, SUITE } 201 \\ & \text { RICHMOND, CALFORNAA 94804-5829 }\end{aligned}$

TELEPHONE: (510) 527-9876

FAX: (510) 527-8164

E-MAIL:MWQ GEOTHERMEX.COM

hydraulically active fractures continues, these simulator features will become more important

than they are now, because there will be a practical basis for their application. 


\section{RECOMMENDATIONS}

Three broad technology areas - Hot Dry Rock, hydrothermal, and nuclear waste isolation - have developed simulation capabilities focused on their immediate needs. The available simulators can be broadly categorized into porous-medium, discrete-fracture, and fracture-network simulators. In each category of simulator, there are several available implementations, none of which can rigorously address the entire range of analytical needs associated with EGS development. Fortunately, it is not necessary to address all of these needs with a single simulator at this time. Instead, simulators can be judiciously selected and applied considering the problem at hand.

As part of this review, the opinions of HDR simulation experts were reviewed. While useful, these discussions highlight the lack of experience with real EGS projects. Thus, many of the evaluations of required EGS features are statements of opinion that are not based on practical experience. At this time, the EGS experience base does not exist to rationally commit to one particular simulator or simulation approach. As such, it is premature to identify one particular simulator as the primary focus of development.

Fracture-based models certainly hold promise. However, the ability to identify and characterize active, discrete fractures is the limiting factor in their effective use. We can apply various characteristics to various fractures in our model, but how well do they represent reality? No EGS reservoir has operated for sufficient time to validate any numerical model, fracture-based or otherwise. Therefore, at the present time, whether or not a particular simulator has the ability to model discrete fractures or to vary the characteristics of those fractures is not hindering the development of EGS.

Developing an EGS simulation experience base should be the highest priority at this time. We strongly believe that meaningful reservoir modeling and simulator development cannot done in the abstract. Meaningful modeling is only done as an active participant in the development and 
TELEPHONE: (510) 527-9876

FAX: (510) 527-8164

E-MAIL: MW@GEOTHERMEX.COM

operation of a reservoir. Only through interaction with realistic problems can the appropriate simulation needs be identified and the skills developed to apply them to other reservoirs.

Therefore, our primary recommendation is that the DOE support active simulation of real EGS reservoirs. This could be done either as part of ongoing international projects, such as those in Japan and Europe, or as part of future EGS development in the US or elsewhere. The active participation of the field developer/operator would be required. We envisage a situation where, for a specific, active project, experts familiar with each of the categories of simulators (porousmedium, discrete- fracture, and fracture-network) would develop models of an EGS. Three (or more) organizations would be funded to apply their technology to simulation of reservoir operation and prediction of future reservoir behavior. The teams would meet regularly to exchange data and concepts. Only this type of "hands-on" experience will provide the background necessary to demonstrate what simulators are appropriate at different stages of reservoir development and what features need to be added to improve the accuracy of the simulation effort. Funding could then be provided to develop these additional features.

At the end of such a project, the field operator(s) would have knowledge of the capabilities of the different simulators. The simulators that perform the best would be favored for similar projects in the future; thus, a combination of real-world experience and market forces (rather than opinions) would decide which approaches are most valuable.

The mathematical basis of fracture-network and discrete-fracture simulators allows the detailed specification of the fracture geometry, which is useful and appropriate when detailed knowledge of in-situ fractures is available. Therefore, we also recommend that further research should be funded for improving both fracture-network simulators and discrete-fracture simulators for EGS use. Potential areas of improvement include the ability to: 1) handle two-phase flow (including experimentation to adapt the cubic law for two-phase conditions); 2) simulate the formation of a hydraulically stimulated fracture network, given appropriate stress information; and 3) modify fracture aperture as a function of both effective and shear stress. 
TELEPHONE: (510) 527-9876

FAX: (510) 527-8164

E-MAIL:MW@GEOTHERMEX.COM

For hydrothermal simulators, the ability to handle rock deformation could be added, which would enable them to be used to model the effect on fracture apertures of : 1) thermal contraction of matrix blocks; 2) changes in effective stress; and 3) changes in shear stress. Furthermore, although the approximation of fractures used in the porous-medium simulators has worked well to model highly fractured geothermal reservoirs, it is cumbersome to implement the specific hydraulic connections associated with a large number of specific fractures. The use of these models to represent discrete, hydraulically active fractures could be investigated further, perhaps by adapting a fracture network generator to the porous-medium solver. The link already developed between FracMan (fracture network mesh generator) and TOUGH2 or FEHM (solvers) could be investigated to determine its utility in EGS evaluations. 
TELEPHONE: $(510)$ 527-9876

FAX: (510) 527-8164

E-MAIL:MW@GEOTHERMEX.COM

\section{REFERENCES}

Antunez, E.U., S.K. Sanyal, A.J. Menzies, T. Naka, R. Takeuchi, S. Iwata, Y. Saeki and T. Inoue, Forecasting Well and Reservoir Behavior Using Numerical Simulation, Uenotai Geothermal Field, Akita Prefecture, Japan. Transactions, Geothermal Resources Council, Vol. 14, 1990.

Antunez, E.U., A.J. Menzies and S.K. Sanyal, Simulating a Challenging Water Dominated Geothermal System: the Cerro Prieto Field, Baja California, Mexico. Proceedings of the 16th Annual Workshop on Geothermal Reservoir Engineering, Stanford University, 1991.

Arihara, S., and N. Arihara, Modeling Silica Deposition in Injection Wells of the Otake Geothermal Field, Japan. Proceedings of the 24th Annual Workshop on Geothermal Reservoir Engineering, Stanford University, 1999.

Barton, N., and V. Choubey, The Shear Strength of Rock Joints in Theory and Practice. Rock Mechanics, Vol. 10, pp. 1-54, 1977.

Barton, C.A., S. Hickman, R. Morin, M. Zoback, T. Finkbeiner, J. Sass, and D. Benoit, Fracture Permeability and Its Relationship to In-Situ Stress in the Dixie Valley, Nevada, Geothermal Reservoir. Proceedings of the 22nd Annual Workshop on Geothermal Reservoir Engineering, Stanford University, 1997.

Battistelli, A., C. Calore, and K. Pruess, The Simulator TOUGH2/EWASG for Modeling Geothermal Reservoirs with Brines and a Non-Condensible Gas. Geothermics, Vol. 26, No. 4, pp. 437-464, 1997.

Biot, M., General Theory of Three-Dimensional Consolidation. Applied Physics, Vol. 12, pp155-164, 1941. 
FAX: (510) 527-8164

E-MAIL: MW@GEOTHERMEX.COM

Board, M., FLAC (Fast Lagrangian Analysis of Continua), Version 2.20. United States Nuclear Regulatory Commission, NUREG/CR-5430, October, 1989.

Boitnott, G.N., Mechanical and transport properties of joints. Ph. D. Thesis, Columbia University, New York, 1991.

Bodvarsson, G.S., Pruess, K., and Lippmann, M. J., Modeling of Geothermal Systems. Journal of Petroleum Technology, September 1986.

Bower, K., A Numerical Model of Hydro-Thermal-Mechanical Coupling in a Fractured Rock Mass. Ph. D. thesis, University of New Mexico, LA-13153-T, UC-403, June 1996.

Brown, S.R., Fluid Flow Through Rock Joints: the Effect of Surface Roughness. Journal of. Geophysical Research, Vol. 92, pp. 1,337-1,347, 1987.

Butler, S. J., D. Benoit, and J.W. Lovekin, A Case History of Numerical Modeling of the Complex Behavior of a Fault-Controlled Geothermal System at Beowawe, Nevada. Proceedings of the World Geothermal Congress 2000, in press.

Carslaw, H.S., and J.C. Jaeger, Conduction of Heat in Solids. Second edition, 396 pp., Clarendon, Oxford, 1959.

Cleary, M.P., and D.T. Barr, Global vs. Local Diffusion Phenomena in Underground Heat and Mass Transfer. Transactions, Geothermal Resources Council, Vol.16, pp. 635-642, 1982.

Cornet, F. H., and R. Jones, Field Evidence on the Orientation of Forced Water Flow With Respect to the Regional Principal Stress Directions. In: First North American Rock Mechanics Symposium, edited by P.P. Nelson and S.E. Laubach, Austin, Texas, pp. 61-69, Balkema, 1994. 
TELEPHONE: (510) 527-9876

FAX: (510) 527-8164

E-MAIL: MW@GEOTHERMEX.COM

Cundall, P. A., Numerical Modeling of Jointed and Faulted Rock. In: Mechanics of Jointed and Faulted Rock, pp. 11-18. Rotterdam: A. A. Balkema, 1990.

DOE Validation Program. Proceedings of the Special Panel on Geothermal Model Intercomparision Study, Proceedings of the 6th Annual Workshop on Geothermal Reservoir Engineering, Stanford University, 1980.

Dash, Z.V., G.A. Zyvoloski and B.A. Robinson, Software Requirements, Design, and Verification and Validation for the FEHM Application - A Finite-Element Heat-and MassTransfer Code, Los Alamos National Laboratory Report LA-13305-MS, UC-800 and UC802, July 1997.

Durham, W.B. and B.P. Bonner, Self-Propping and Fluid Flow in Slightly Offset Joints at High Effective Confining Pressures. Journal of Geophysical Research, 99, 9391-9399, 1994.

DuTeaux, R. and T.J. Callahan, Comparing Reactive and Non-Reactive Tracers to Measure Changes in Liquid-Dominated, Fractured Geothermal Reservoirs. Transactions, Geothermal Resources Council, Vol. 20, pp. 447-451, 1996.

Edwards, A.L., TRUMP: A Computer Program for Transient and Steady State Temperature Distributions in Multidimensional Systems. National Technical Information Services, National Bureau of Standards, Springfield, VA, 1972.

Eguchi, Y., T. Yamamoto, K. Kitano, T. Nishihara, and H. Ohnishi, Three-Dimensional Computation of Velocity and Pressure Fields in the Ogachi HDR Reservoir. Proceedings of the $4^{\text {th }}$ International HDR Forum, Strasbourg, September 28-30, 1998a.

Eguchi, Y., et al., Visualization of Deep Underground for Hot-Dry-Rock Power Generation. Computer Visualization Symposium, Tokyo, pp.21-24, 1998 b. 
TELEPHONE: (510) 527-9876

FAX: (510) 527-8164

E-MAIL: MW@GEOTHERMEX.COM

Evans, K.F., T. Kohl, R.J. Hopkirk, and L. Rybach, Modelling of energy production from Hot Dry Rock systems. Final Report to Swiss National Energy Research Fund, Swiss Federal Institute of Technology/Polydynamics, NEFF 359, April, 1992.

Friedly, J.C., and J. Rubin, Solute Transport with Multiple Equilibrium Controlled or Kinetically Controlled Chemical Reactions. Water Resources Research, Vol. 28, pp. 1,935$1,953,1992$.

Gangi, A.F., Variation of Whole and Fractured Porous Rock Permeability with Confining Pressure. Int. J. Rock Mech. Min. Sci. \& Geomech Abstr., Vol 15, pp. 249-257, 1978.

GERD, FY 1995 Summary of Hot Dry Rock Geothermal Power Project in Japan. New Energy and Industrial Technology Development Organization, Tokyo, Japan, 1996.

GERD, FY 1996 Summary of Hot Dry Rock Geothermal Power Project in Japan. New Energy and Industrial Technology Development Organization, Tokyo, Japan, 1997.

Glover, P.W.J., K. Matsuki, R. Hikima and K. Hayashi, Fluid Flow in Fractally Rough Synthetic Fractures. Geophysical Research Letters, to appear 1996.

Goodman, R. E., Methods of Geological Engineering in Discontinuous Rocks. West Publishers, St. Paul, Minnesota, 1976.

Hadgu, T., R.W. Zimmerman, and G.S. Bodvarsson, Coupled Reservoir-Wellbore Simulation of Geothermal Reservoir Behavior. Geothermics, Vol. 24, No. 2, pp. 145-166, 1995.

Hakami, E., Aperture Measurements and Flow Experiments on a Single Natural Fracture. Int. J. Rock Mech. Min. Sci. \& Geomech. Abst., 33, 395-404, 1996. 
TELEPHONE: (510) 527-9876

FAX: (510) 527-8164

E-MAIL: MW@GEOTHERMEX.COM

Hardeman, B. and D. Swenson, A Geometric Modeling Framework for the Numerical Analysis of Geothermal Reservoirs. Proceedings of the 23rd Annual Workshop on Geothermal Reservoir Engineering, Stanford University, 1998.

Hudson, J. A. (Ed.), Special Issue on Thermo-Hydro-Mechanical Coupling. Int. J. of Rock Mechanics and Mining Sciences, Vol. 32, No. 5, 1995.

Hughes, Thomas J.R., The Finite-element Method. Prentice-Hall, Inc., Englewood Cliffs, New Jersey, 1987.

Jing, Zhenzi, Simulation of Heat Extraction from Fractured Geothermal Reservoirs. Report from Ph.D. thesis, Department of Machine Intelligence and System Engineering, Faculty of Engineering, Tohoku University, Sendai, Japan, 1998.

Jing, Z., J. Willis-Richards, K. Watanabe, and T. Hashida, A New 3-D Stochastic Model for HDR Geothermal Reservoir in Fractured Crystalline Rock. Proceedings of the $4^{\text {th }}$ International HDR Forum, Strasbourg, September 28-30, 1998.

Jones, R.H., A. Beuce, A. Jupe, H. Fabriol, and B.C. Dyer, Imaging Induced Seismicity During the 1993 Injection Test at Soultz-sous-Forets, France. Proceedings of the World Geothermal Congress, Florence, Italy, pp. 2,665-2,669, 1995.

Kohl, T., K.F. Evans, R.J. Hopkirk, and L. Rybach, Modelling of Coupled Hydraulic, Thermal and Mechanical Processes in the Simulation of Hot Dry Rock Reservoir Behaviour. In: Conference on Fractured and Jointed Rock Masses, edited by L. Myer et al., Lake Tahoe, California, pp. 535-542, Balkema, Rotterdam, 1992.

Kohl, T., K.F. Evans, R.J. Hopkirk, and L. Rybach, Coupled Hydraulic, Thermal, and Mechanical Considerations for the Simulation of Hot Dry Rock Reservoirs. Geothermics, Vol. 24, No. 3, pp. 345-359, 1995. 
Kohl, T., et al., Observation and Simulation of Non-Darcian Flow Transients in Fractured Rock. Water Resources Research, Vol. 33, No. 3, pp. 407-418, March 1997.

Kohl, T., Coupled 3-D Simulations of Long Term HDR Behavior. Draft Report, November 1998.

Lardanyi, B., and G. Archambault, Simulation of Shear Behavior of a Jointed Rock Mass. Proceedings of the $11^{\text {th }}$ Symposium on Rock Mechanics, AIME, New York, pp. 105-125, 1970.

Lichtner, P., Time-Space Continuum Description of Fluid/Rock Interaction in Permeable Media. Water Resources Research, Vol. 12, pp. 3,135-3,155, 1976.

Lichtner, P.C. and M.S. Seth, Multiphase-Multicomponent Non-isothermal Reactive Transport in Partially Saturated Porous Media. Presented at the International Conference on Geological Disposal of Radioactive Waste, Canadian Nuclear Society, Sept. 16-19, Winnipeg Manitoba, Canada, 1996.

Matsuki, K., K. Sakaguchi and S. Li, Fractal Nature of Crack Surfaces, Characteristics of Fluid Flow Through a Crack and Interfacial Stiffness Induced by Contact Between Two Crack Surfaces. Presented at the Symposium on Fractal Fracture Mechanics and Advanced Geothermal Energy Extraction System Design, Tohoku University, Sendai, Japan, 1996.

Menzies, A.J., E.E. Granados, S.K. Sanyal, L. Mérida-I. and A. Caicedo-A., Numerical Modeling of the Initial State and Matching of Well Test Data from the Zunil Geothermal Field, Guatemala. Proceedings of the 16th Annual Workshop on Geothermal Reservoir Engineering, Stanford University, 1991.

Molloy, M.W., Geothermal Reservoir Engineering Code-Comparison Project. Proceedings of the 6th Annual Workshop on Geothermal Reservoir Engineering, Stanford University, 1980. 


\section{GeothermEx, Inc.}

FAX: (510) 527-8164

E-MAIL:MW@GEOTHERMEX.COM

Narasimhan, T.N., and P.A. Witherspoon, An Integrated Finite-difference Method for Analyzing Fluid Flow in Porous Media, Water Resources Research, Vol. 12, No. 1, pp. 57-64, 1976.

Pine, R.J., and A.S. Batchelor, Downward Migration of Shearing in Jointed Rock During Hydraulic Injections. Int. J. Rock Mech., Min. Sci. \& Geomech. Abstr., 21, 249-263, 1984.

Pritchett, J.W., The DOE Code-Comparison Study: Summary of Results for Problem 5. Proceedings of the 6th Annual Workshop on Geothermal Reservoir Engineering, Stanford University, 1980.

Pritchett, J. W., STAR: A Geothermal Reservoir Simulation System. Proceedings of the World Geothermal Congress, Vol. 4, pp. 2,959-2,963, 1995.

Pruess, K., Modeling of Geothermal Reservoirs: Fundamental Processes, Computer Simulation, and Field Applications. Geothermics, Vol. 19, No. 1, pp. 3-15, 1990.

Pruess, K., TOUGH2 - A General Purpose Numerical Simulator for Multiphase Fluid and Heat Flow. Lawrence Berkeley National Laboratory Report LBL-29400, 1991.

Pruess, K., C. Oldenburg, and G. Moridis, Overview of TOUGH2, Version 2.0. Proceedings of the TOUGH Workshop '98, pp. 307-315, 1998.

Schrauf, T.W., and D.D. Evans, Laboratory Studies of Gas Flow Through a Single Natural Fracture. Water Resources Research, Vol. 22, pp.1,038-1,050, 1986.

Steefel, C.I., and A.C., Lasaga, A Coupled Model for Transport of Multiple Chemical Species and Kinetic Precipitation/Dissolution Reactions with Applications to Reactive Flow in Single Phase Hydrothermal Systems. American Journal of Science, 294, pp. 529-592, 1994. 
TELEPHONE: (510) 527-9876

FAX: (510) 527-8164

E-MAIL: MW@GEOTHERMEX.COM

Structured Academic Review of HDR/HWR, "Engineered Geothermal Systems: How We Think Reservoirs Work and What To Do Next," Tohoku University, Sendai, Japan, March 14-16, 1997.

Swenson, D., User's Manual for Geocrack2D: A Coupled Fluid Flow/Heat Transfer/Rock Deformation Program for Analysis of Fluid Flow in Jointed Rock. Manual Release 3.11b, Kansas State University, 1997a.

Swenson, D. and B. Hardeman, The Effects of Thermal Deformation on Flow in a Jointed Geothermal Reservoir. 36th U.S. Rock Mechanics Symposium, ISRM International Symposium, New York, NY, June 29-July2, $1997 \mathrm{~b}$.

Swenson, D., R. Schroeder, N. Shinohara and T. Okabe, Analyses of the Hijiori Long Term Circulation Test. Proceedings of the 24th Annual Workshop on Geothermal Reservoir Engineering, Stanford University, 1999.

Tezuka, K., K. Watanabe and M. Miyairi, Deterministic and Stochastic Approach to Building Fracture Network Model of Hijiori HDR Reservoir. Proceedings of the $4^{\text {th }}$ International HDR Forum, Strasbourg, September 28-30, 1998.

Torgersen, T., Crustal Scale Fluid Transport: Magnitude and Mechanism. Geophys. Res. Lett., Vol. 18, pp. 917-918, 1991.

Tsang, C.F. (Editor), Coupled Processes Associated With Nuclear Waste Repositories. Academic Press, ISBN 0127016201.801 pp., 1987.

Tsang, C.F., Coupled Hydromechanical-Thermomechanical Processes in Rock Fractures. Reviews of Geophysics, Vol. 29, No. 4, pp. 537-551, 1991

Warren, J.E., and P.J. Root, The Behavior of Naturally Fractured Reservoirs. Society of Petroleum Engineers, Transactions, AIME, Vol. 228, pp. 245-255, 1963. 
White, S.P., Multiphase Non-Isothermal Transport of Systems of Reacting Chemicals. Water Resources Research, Vol. 31, pp. 1761-1772, 1995.

White, S.P., T. Xu, and K. Pruess, Reactive Chemical Transport. Proceedings of the TOUGH Workshop '98, pp. 244-249, 1998.

Willis-Richards, J., A.S.P. Green, and A. Jupe, A Comparison of HDR Geothermal Sites. Proceedings of the World Geothermal Congress, pp. 2,601-2,605, 1995.

Willis-Richards, J. and T. Wallroth, Approaches to the Modeling of HDR Reservoirs - A Review. Geothermics, Vol. 24, No. 3, pp. 307-332, 1995.

Willis-Richards, J., K. Watanabe and H. Takahashi, Progress Toward a Stochastic Rock Mechanics Model of Engineerd Geothermal Systems. Journal of Geophysical Research, Vol. 101, Np. B8, pp. 171,481-171,496, 1996.

Witherspoon, P.A., J.S.Y. Wang, K. Iwai, and J.E. Gale, Validity of the Cubic Law for Fluid Flow in a Deformable Rock Fracture. Water Resources Research, Vol. 16, pp. 1,016-1,024, 1980.

Yamamoto, T., Y. Fujimitsu and H. Ohnishi, Hot Dry Rock Reservoir 3D Simulation of the Ogachi Site. Transactions, Geothermal Resources Council, Vol. 19, pp. 287-294, 1995.

Yamamoto, T., K. Kitano, Y. Fujimitsu and H. Ohnishi, Application of Simulation Code, GEOTH3D, on the Ogachi HDR Site. Proceedings of the 22rd Annual Workshop Geothermal Reservoir Engineering, Stanford University, January 27-29, 1997.

Yamamoto, T., et al., Application of Simulation Code, GEOTH3D, to the Fenton Hill HDR Site. Proceedings of the 23rd Annual Workshop Geothermal Reservoir Engineering, Stanford University, January 26-28, 1998. 
TELEPHONE: (510) 527-9876

FAX: (510) 527-8164

E-MAIL:MW@GEOTHERMEX.COM

$\mathrm{Xu}, \mathrm{T} ., \mathrm{K}$. Pruess, and G. Brimhall, Introducing Reactive Chemical Transport to TOUGH2:

Application to Supergene Copper Enrichment, Proceedings of the TOUGH Workshop '98, pp. 234-243, 1998.

Zhao, J., and E. T. Brown, Thermal Cracking Induced by Water Flow Through Joints in Heated Granite. Int. J. Rock Mech. Min. Sci. \& Geomech. Abstr., 29, 77-82, 1992.

Zyvoloski, G.A., B.A. Robinson, Z.V. Dash and L.L. Trease, User's Manual for the FEHM Application - A Finite-Element Heat- and Mass-Transfer Code, Los Alamos National Laboratory Report LA-13306-M, UC-800 and UC-802, July 1997. 


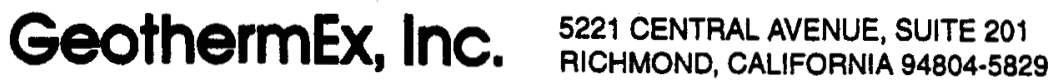

TELEPHONE: $(510)$ 527.9876

FAX: $(510)$ 527-8164

E-MAIL: GEOTHERMEX OCOMPUSERVE.COM

\section{TABLES}


Table 1: Features of HDR Simulators Potentially Applicable to EGS

\begin{tabular}{|c|c|c|c|c|}
\hline Capability & HRAO Iure & GHollo & Ir. & 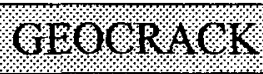 \\
\hline Discrete fractures & - & - & 1 & - \\
\hline Aperture function of normal stress & - & & 2 & - \\
\hline Aperture function of shear & & & $\bullet$ & \\
\hline Flow rate as function of aperture & 3 & & $\bullet$ & - \\
\hline Channeling & 4 & & & \\
\hline Porous flow in matrix & - & - & & \\
\hline Thermo-elastic effects & - & & 2 & - \\
\hline Tracer transport & - & & • & - \\
\hline \multicolumn{5}{|l|}{ Multi-phase flow } \\
\hline $3 \mathrm{D}$ & - & - & $\bullet$ & \\
\hline Irregular grid & & & - & - \\
\hline Mineral deposition / dissolution & & & - & \\
\hline
\end{tabular}

1) Discrete fractures during stimulation, converted to equivalent porous media for operation analysis

2) Based on global stress, no local elasticity solution

3) Includes laminar and turbulent flow laws

4) Can be implemented by manually adjusting fracture element properties 
Table 2: Features of Hydrothermal Simulators Potentially Applicable to EGS

\begin{tabular}{|c|c|c|c|c|}
\hline (Capability. & FHIIM & STIAR & TMORHO & 1010912 \\
\hline Discrete fractures & $\bullet$ & $\bullet$ & - & - \\
\hline \multicolumn{5}{|c|}{ Aperture function of normal stress } \\
\hline \multicolumn{5}{|l|}{ Aperture function of shear } \\
\hline \multicolumn{5}{|l|}{ Flow rate function of aperture } \\
\hline \multicolumn{5}{|l|}{ Channeling } \\
\hline Porous flow in matrix & $\bullet$ & $\bullet$ & $\bullet$ & $\bullet$ \\
\hline Thermo-elastic effects & - & - & - & $\bullet$ \\
\hline Tracer transport & - & - & $\bullet$ & $\bullet$ \\
\hline Multi-phase flow & $\bullet$ & $\bullet$ & $\bullet$ & - \\
\hline $3 \mathrm{D}$ & - & - & - & - \\
\hline Irregular grid & - & & & $\bullet$ \\
\hline Mineral deposition/ dissolutio & & & & \\
\hline
\end{tabular}

1) Discrete fractures during stimulation, converted to equivalent porous media for operation analysis

2) Based on global stress, no local elasticity solution

3) Includes laminar and turbulent flow laws

4) Can be implemented by manually adjusting fracture element properties 
Table 3: Features of Nuclear-Waste-Isolation Simulators Potentially Applicable to EGS

\begin{tabular}{|c|c|c|c|c|}
\hline Capability. & (ASTIUM 2001) & (FIST) & 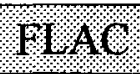 & 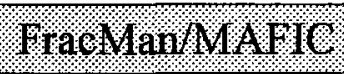 \\
\hline Porosity: Single (1) or Dual (2) & 1 & 1 & 1 & $1-2$ \\
\hline Model Dimension & $1-2$ & $1-2-3$ & $1-2$ & 3 \\
\hline Discrete Fracture Networks & no & no & no & yes \\
\hline Matrix Permeability & yes & yes & yes & yes \\
\hline Thernmal (T) - Hydro $(\mathrm{H})$ - Mechanical (M) & T-H-M & $\mathrm{T}-\mathrm{H}$ & T-H-M & T-H-M \\
\hline Aperture/Transmissivity Coupling & yes & no & - & indirect \\
\hline Aperture/Stress Coupling & yes & no & -- & indirect \\
\hline Channeling & no & no & no & yes \\
\hline Thermo-Elastic Effects & yes & no & yes & indirect \\
\hline Tracer Transport & no & yes & no & yes \\
\hline Multi-Phase Flow & no & no & no & no \\
\hline Vapor/Water Phase Transitions & no & no & no & no \\
\hline Irregular Grid (i.e., Finite Element) & yes & yes & yes & yes \\
\hline Mineral Deposition / Dissolution & no & no & no & no \\
\hline
\end{tabular}


Table 3: Features of Nuclear-Waste-Isolation Simulators Potentially Applicable to EGS

\begin{tabular}{|c|c|c|c|c|}
\hline Sapavility. & HOORTI & W. YUYMO & WOS11 & 1. 114 \\
\hline Porosity: Single (1) or Dual (2) & 2 & $1-2$ & 1 & 1 \\
\hline Model Dimension & 2 & 2 & 3 & $1-2-3$ \\
\hline Discrete Fracture Networks & yes & yes & yes & no \\
\hline Matrix Permeability & yes & yes & yes & yes \\
\hline Thermal (T) - Hydro $(\mathrm{H})$ - Mechanical (M) & T-H-M & T-H-M & T-H-M & $\mathrm{T}-\mathrm{H}$ \\
\hline Aperture/Transmissivity Coupling & yes & yes & yes & no \\
\hline Aperture/Stress Coupling & yes & yes & yes & no \\
\hline Channeling & yes & no & no & no \\
\hline Thermo-Elastic Effects & yes & yes & yes & no \\
\hline Tracer Transport & no & no & yes & yes \\
\hline Multi-Phase Flow & no & no & no & yes \\
\hline Vapor/Water Phase Transitions & no & no & no & no \\
\hline Irregular Grid (i.e., Finite Element) & yes & yes & yes & yes \\
\hline Mineral Deposition / Dissolution & no & no & no & no \\
\hline
\end{tabular}

Table 3, Page 2 of 4 
Table 3: Features of Nuclear-Waste-Isolation Simulators Potentially Applicable to EGS

\begin{tabular}{|c|c|c|c|c|}
\hline Capability & PHOHNISS & PORHIO & ROY MAS & slorre \\
\hline Porosity: Single (1) or Dual (2) & $1-2$ & $1-2$ & 1 & 1 \\
\hline Model Dimension & 3 & 3 & $\overline{2}$ & 2 \\
\hline Discrete Fracture Networks & yes & no & yes & no \\
\hline Matrix Permeability & yes & yes & yes & yes \\
\hline Thermal $(\mathrm{T})$ - Hydro $(\mathrm{H})$ - Mechanical $(\mathrm{M})$ & T-H-M & $\mathrm{T}-\mathrm{H}$ & T-H-M & $\mathrm{T}-\mathrm{H}$ \\
\hline Aperture/Transmissivity Coupling & yes & no & yes & no \\
\hline Aperture/Stress Coupling & yes & no & yes & no \\
\hline Channeling & no & no & no & no \\
\hline Thermo-Elastic Effects & yes & no & yes & no \\
\hline Tracer Transport & yes & yes & no & no \\
\hline Multi-Phase Flow & yes & yes & no & no \\
\hline Vapor/Water Phase Transitions & no & yes & no & no \\
\hline Irregular Grid (i.e., Finite Element) & no & no & yes & yes \\
\hline Mineral Deposition / Dissolution & no & yes & no & mo \\
\hline
\end{tabular}

Table 3, Page 3 of 4 
Table 3: Features of Nuclear Waste Isolation Simulators Potentially Applicable to EGS

\begin{tabular}{|c|c|c|c|c|}
\hline Capability. & SWIFI98 & THAMISs & 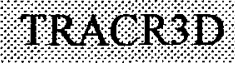 & Y1010. \\
\hline Porosity: Single (1) or Dual (2) & 1 & 1 & $1-2$ & 1 \\
\hline Model Dimension & $1-2-3$ & 3 & 3 & $2-3$ \\
\hline Discrete Fracture Networks & no & no & no & yes \\
\hline Matrix Permeability & yes & yes & yes & no \\
\hline Thermal $(\mathrm{T})$ - Hydro $(\mathrm{H})$ - Mechanical & T-H & T-H-M & $\mathrm{T}-\mathrm{H}$ & T-H-M \\
\hline Aperture/Transmissivity Coupling & no & no & no & yes \\
\hline Aperture/Stress Coupling & no & no & no & yes \\
\hline Channeling & no & no & no & no \\
\hline Thermo-Elastic Effects & no & yes & no & yes \\
\hline Tracer Transport & yes & no & yes & no \\
\hline Multi-Phase Flow & no & no & yes & no \\
\hline Vapor/Water Phase Transitions & no & no & yes & no \\
\hline Irregular Grid (i.e., Finite Element) & no & no & yes & yes \\
\hline Mineral Deposition / Dissolution & no & no & no & no \\
\hline
\end{tabular}


TELEPHONE: $(510) 527.9876$

FAX: (510) 527-8164

E-MAIL: GEOTHERMEX COMPUSERVE:COM

\section{FIGURES}




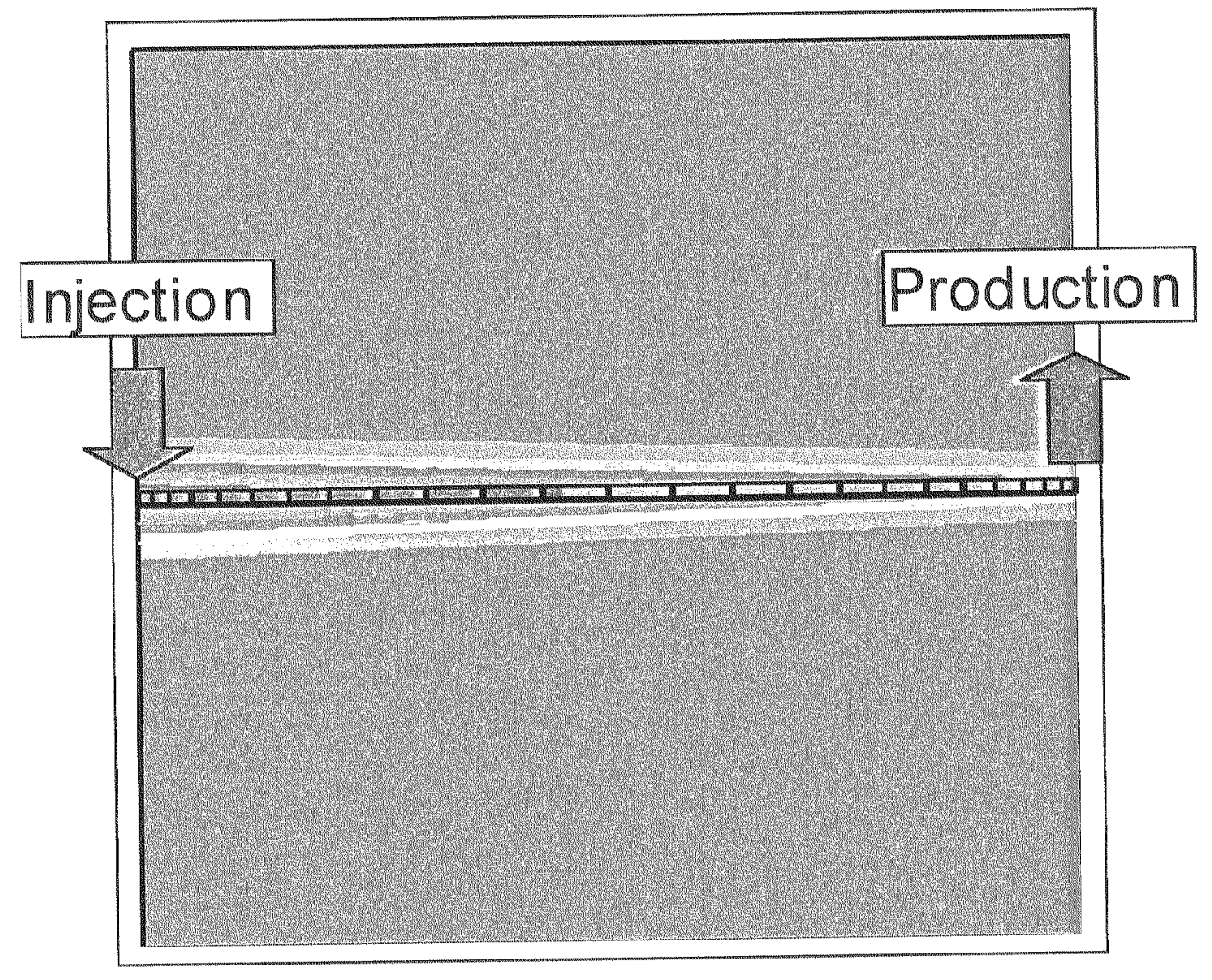

Figure 1: Cooling on surface of fracture 


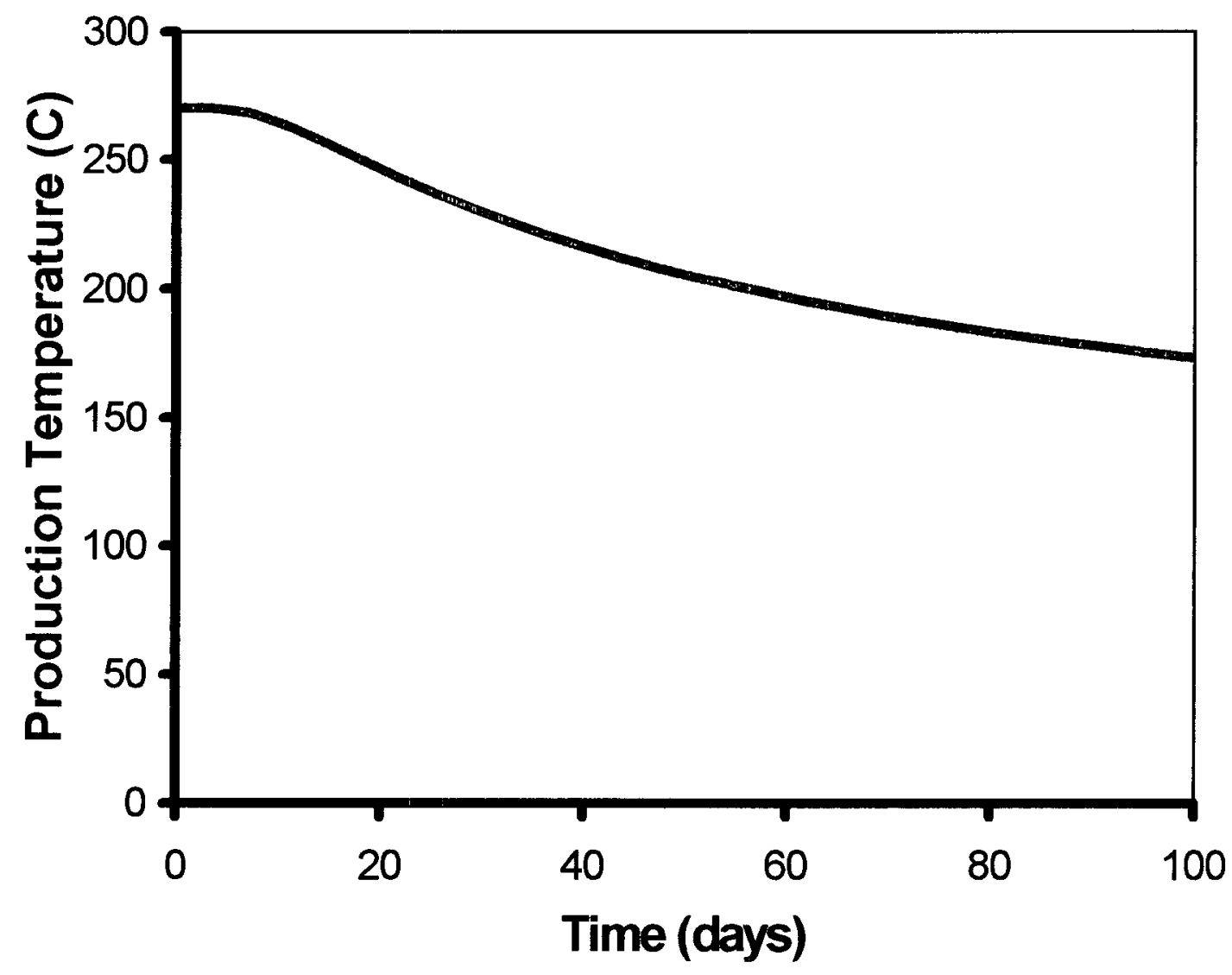

Figure 2: Cooling at production well as a result of fracture surface cooling 


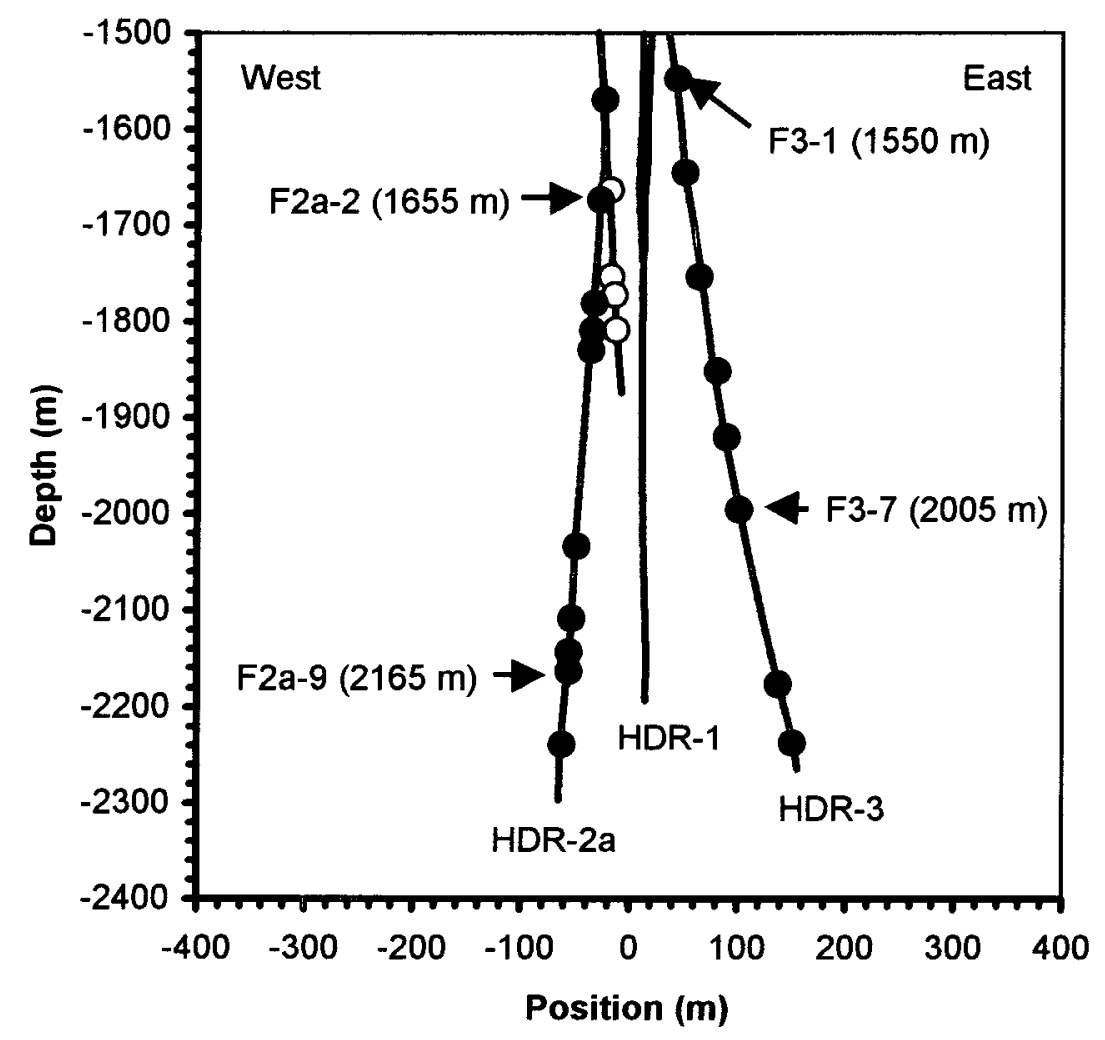

Figure 3: Vertical section of Hijiori reservoir looking north 


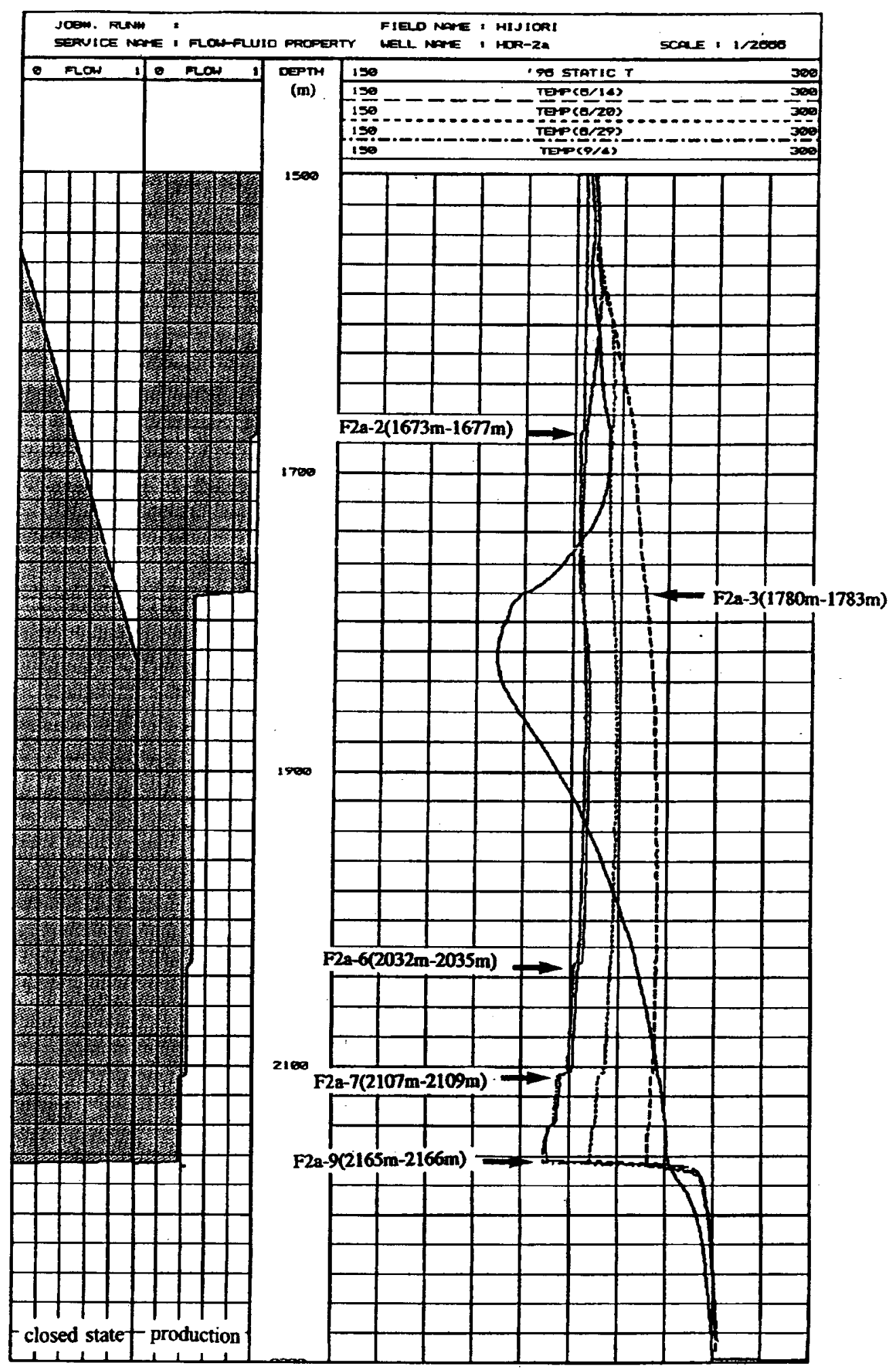

Figure 4: Flow rates and temperature measurement in HDR-2a (from GERD, 1997) 


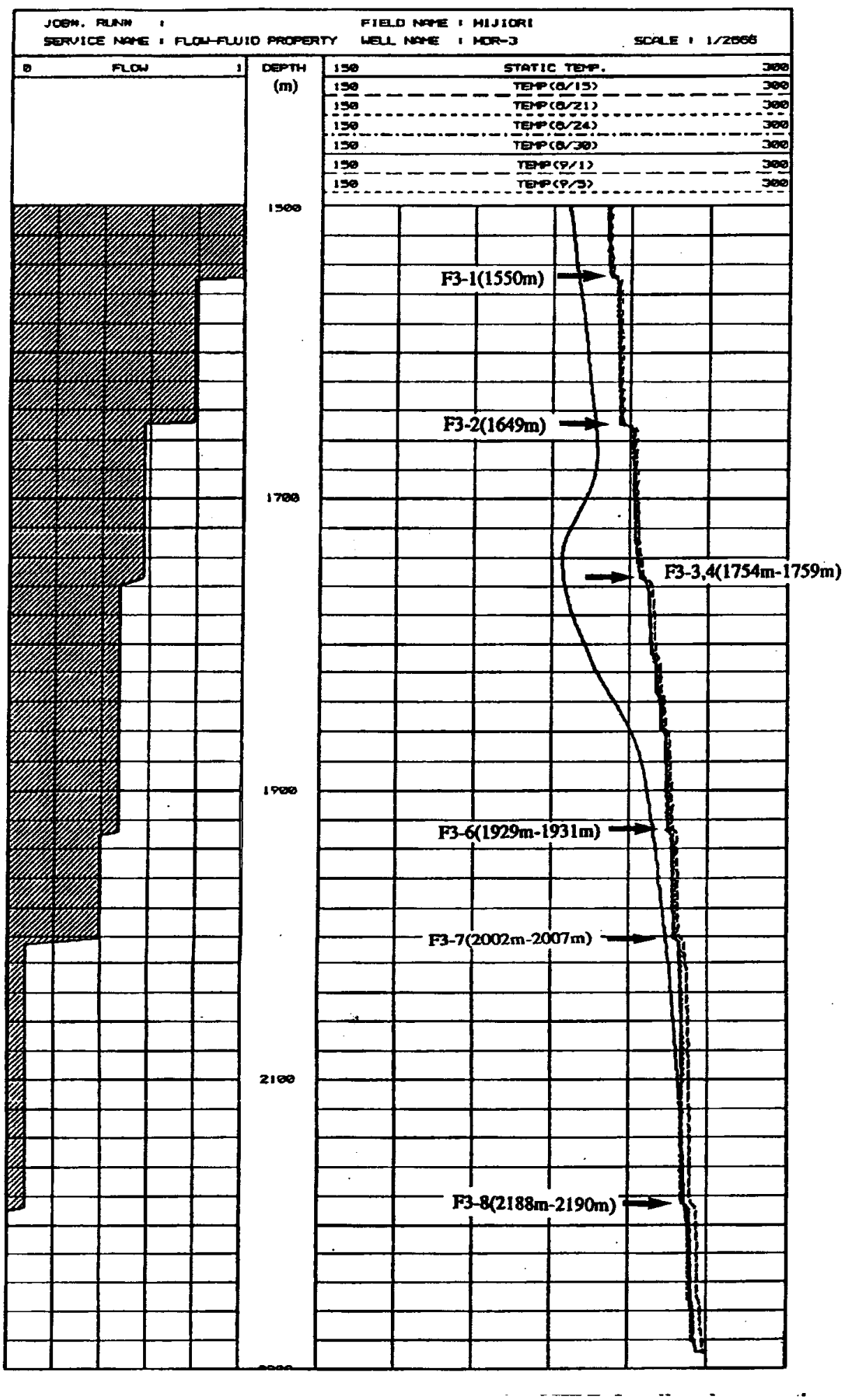

Figure 5: Flow rates and temperature measurement in HDR-3 (from GERD, 1997) 

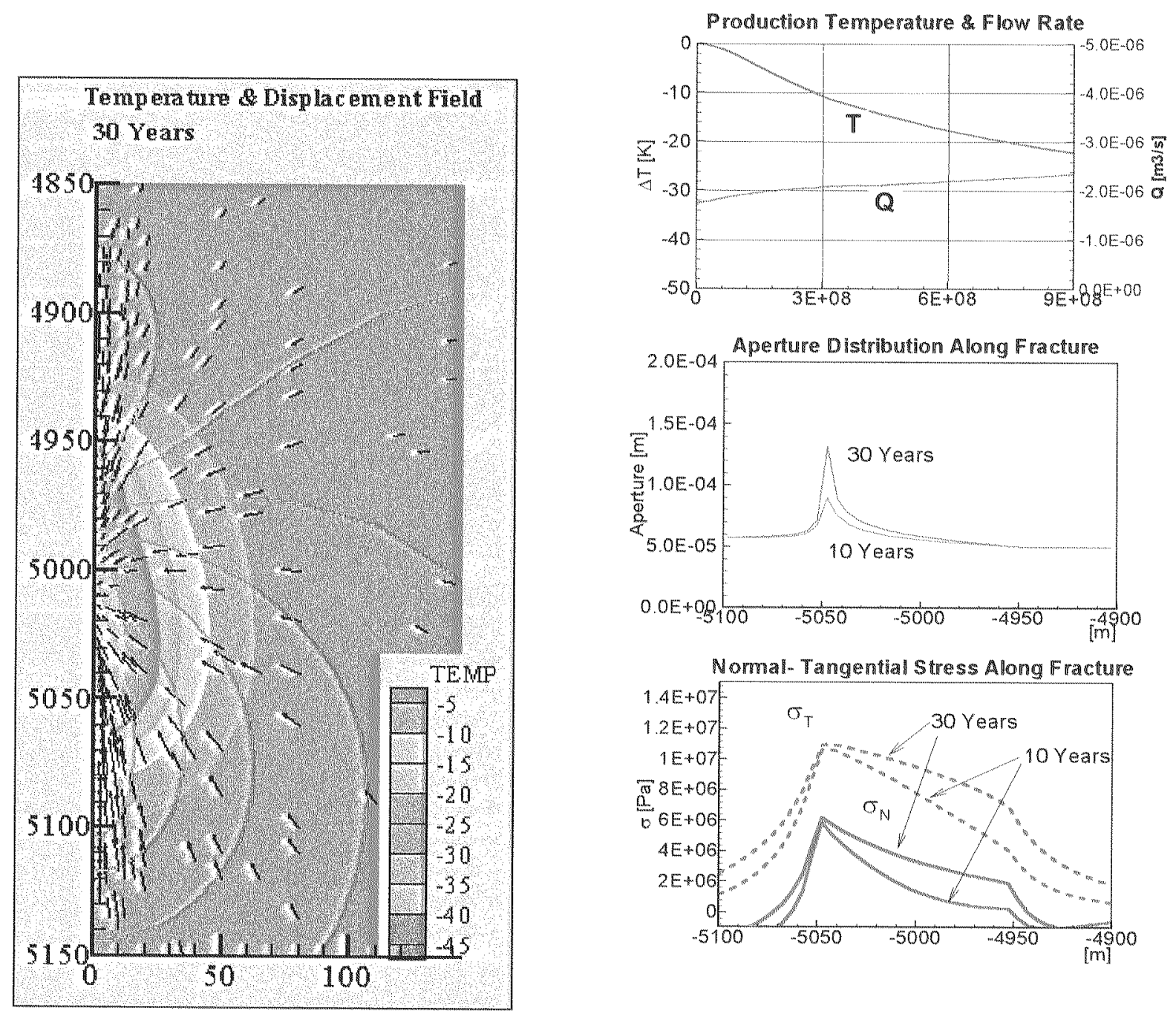

Figure 6: Results of the 2-D single fracture geometry run. The temperature and hydraulic pressure fields (background and white contour lines) are shown in the left frame. To the right, from top to bottom respectively, are shown the temperature and flow rate histories, the aperture distributions along the fracture surface, and the profiles describing the stress perturbation within the host rock along the fracture surface. (Kohl, 1998) 

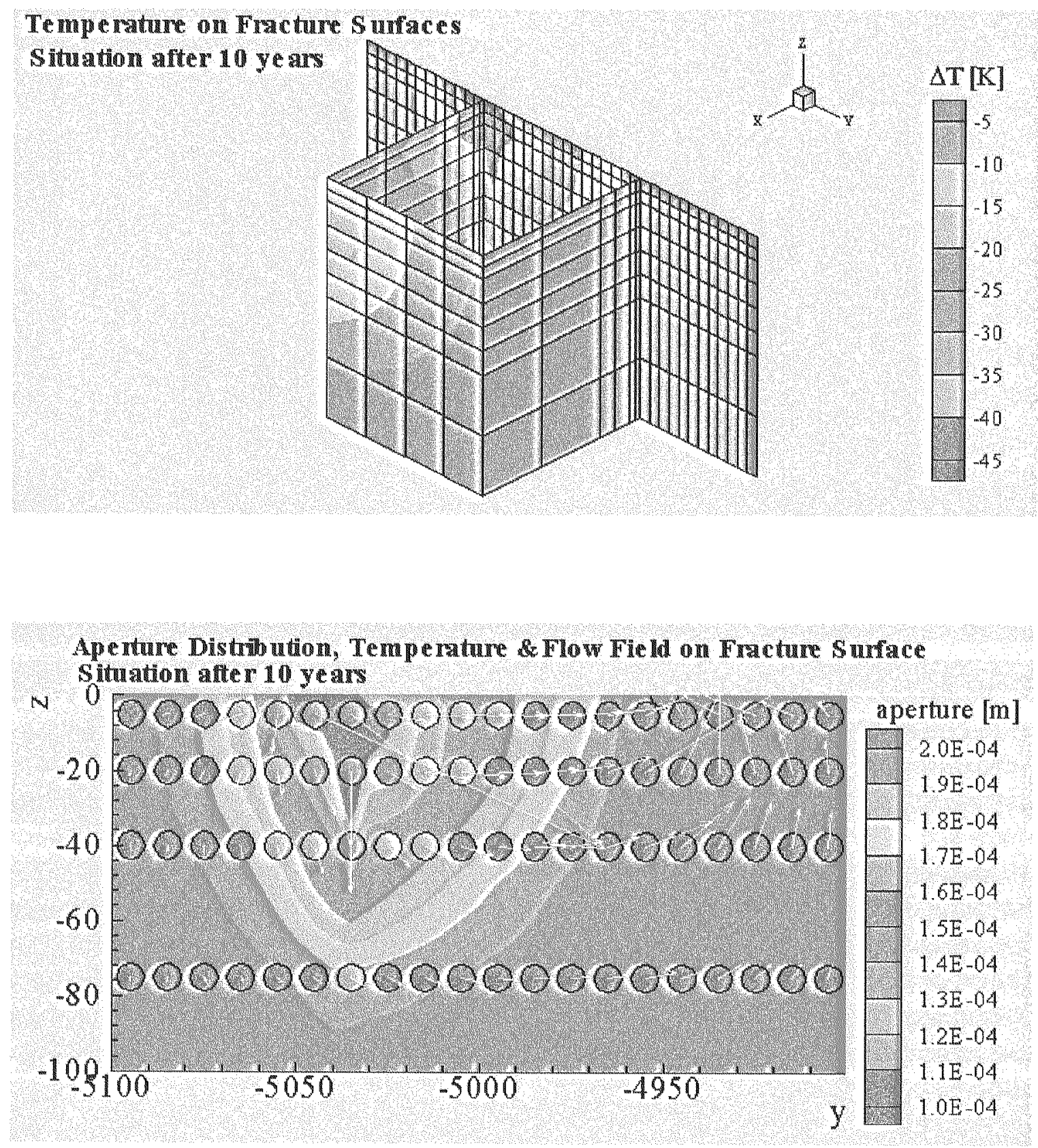

Figure 7: Results of the 3-D multiple fracture geometry run. Initial apertures of 100 $\mu \mathrm{m}$ were assigned to the primary flow path and $200 \mu \mathrm{m}$ to the other fractures. The upper frame shows the temperature distribution in the four fractures after 10 years 


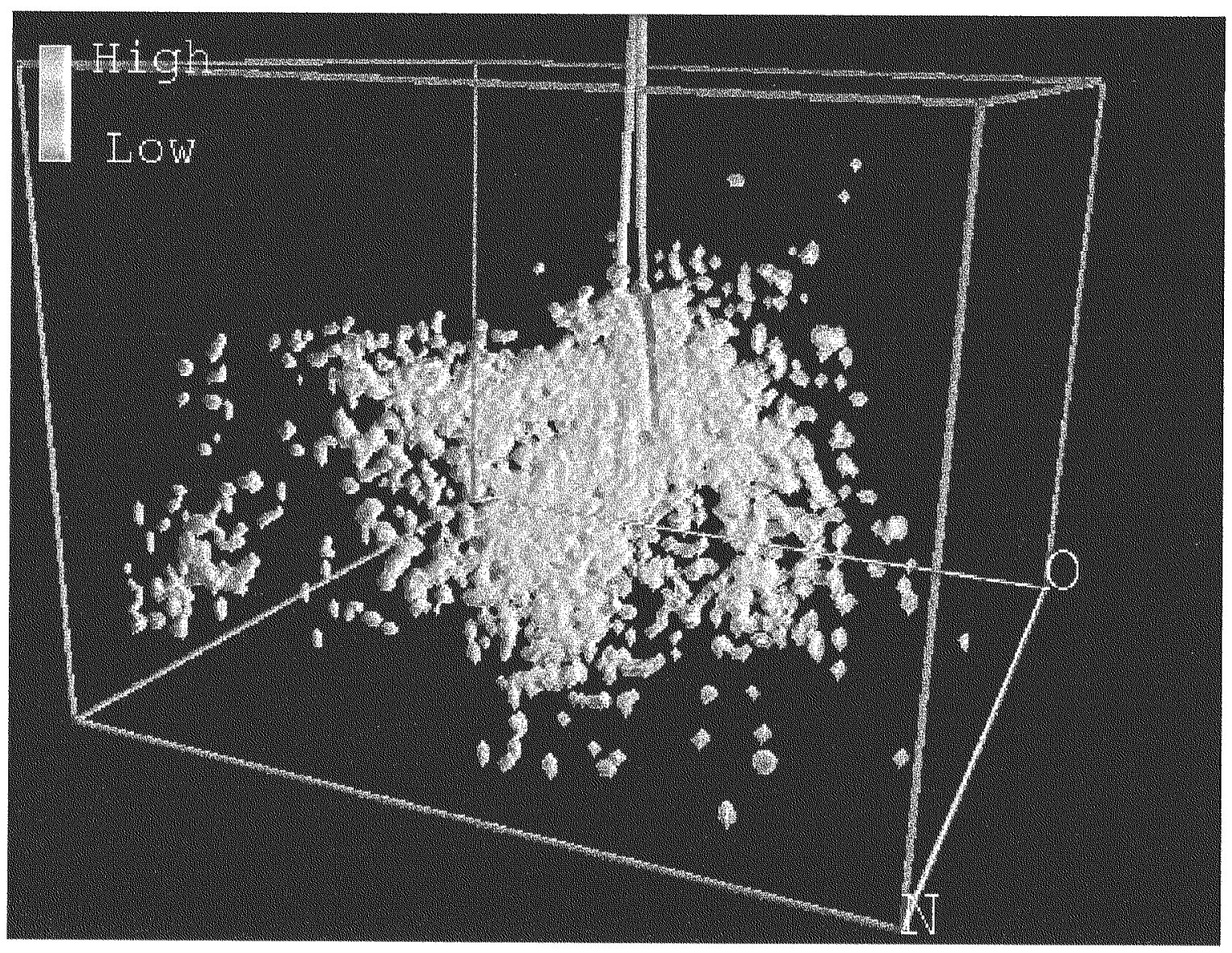

Figure 8: Example showing permeability distribution

((c) Y.Eguchi et al., 1998b) 


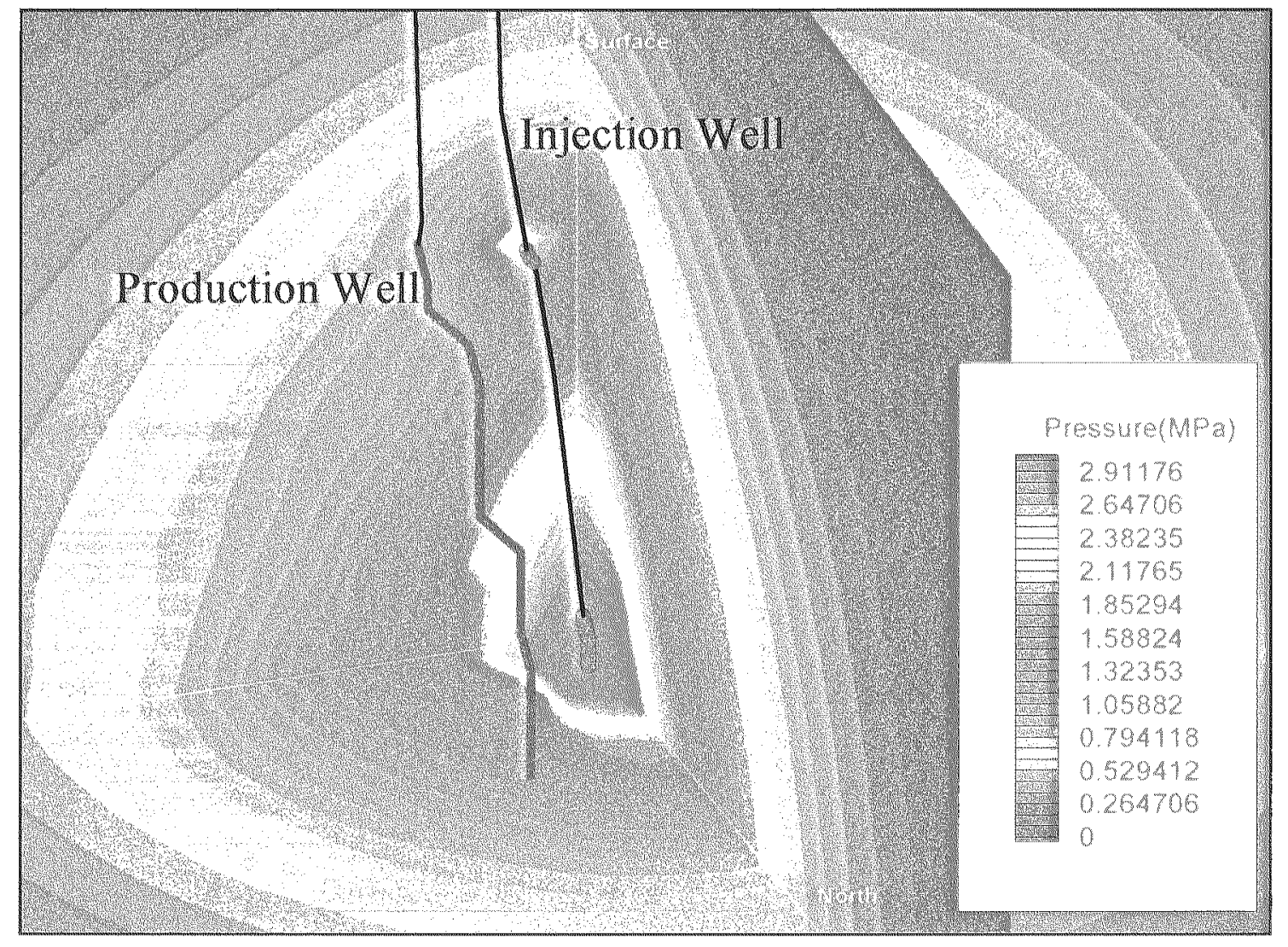

Figure 9: Resulting pressure contour and flow vectors at $3430 \mathrm{~m}$ depth (Eguchi et al., 1998a) 


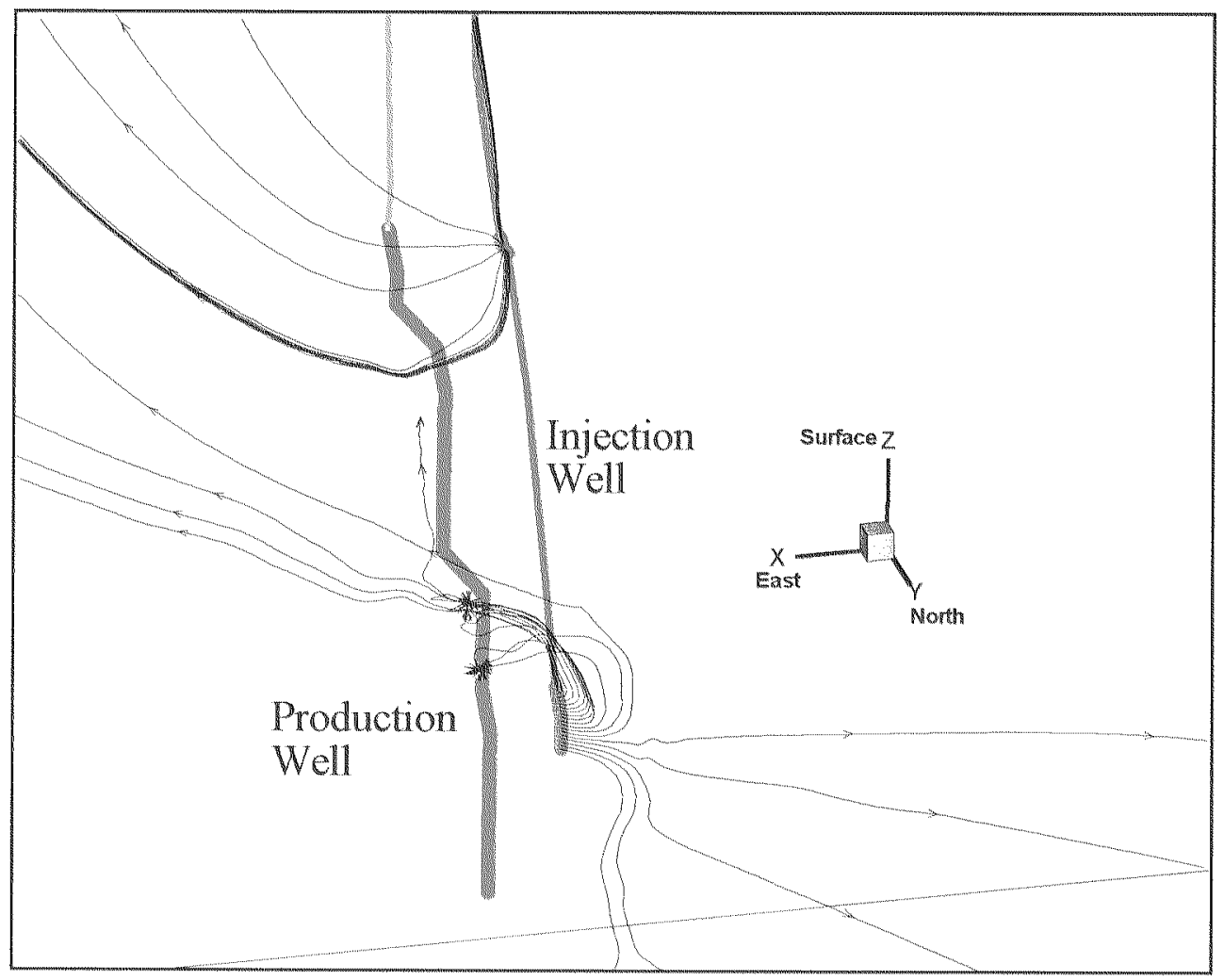

Figure 10: Streamline originating from the two injection open hole intervals (Eguchi et al., 1998a) 


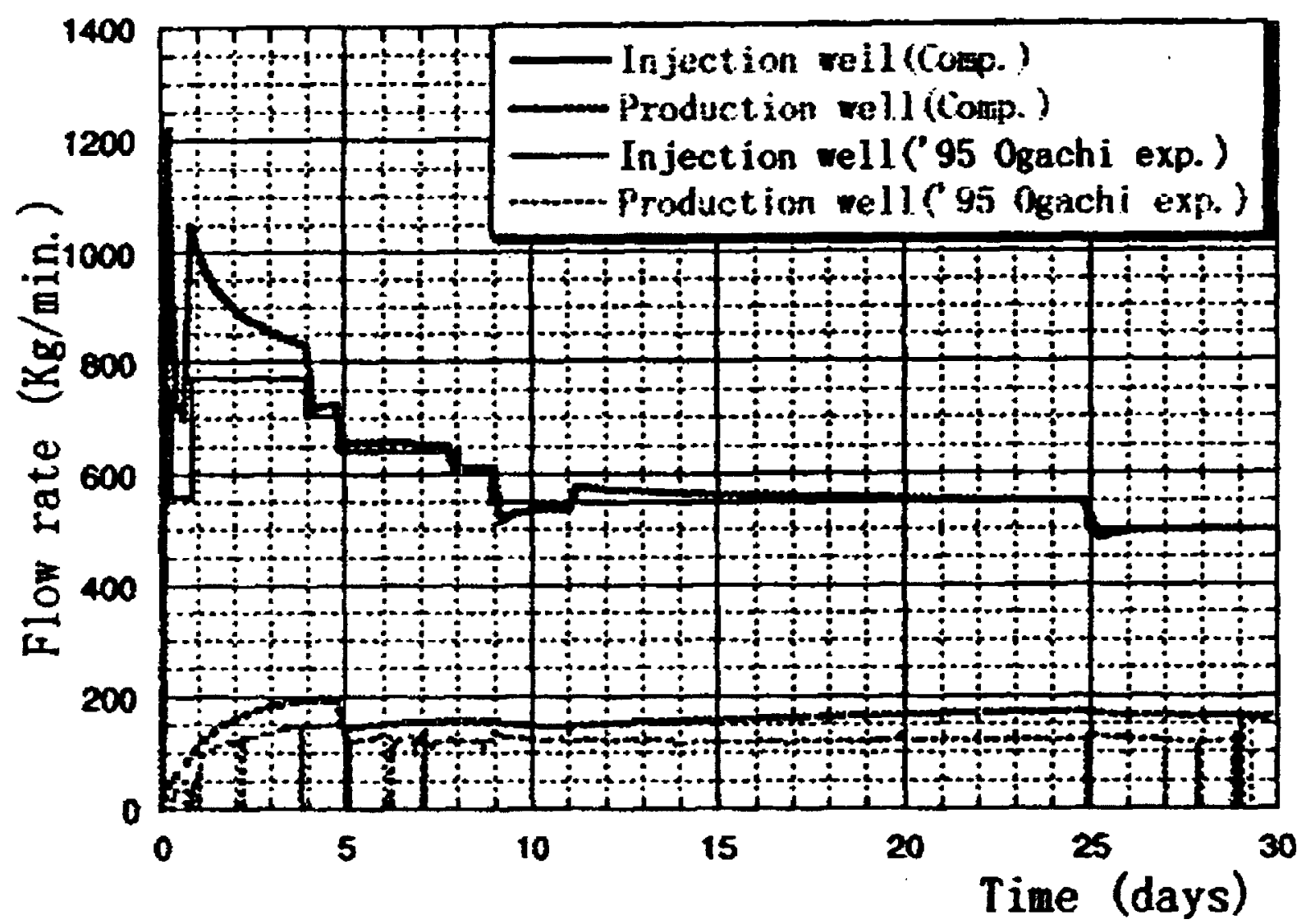

Figure 11: Time histories of the computed and measured flow rates (Eguchi et al., 1998a) 


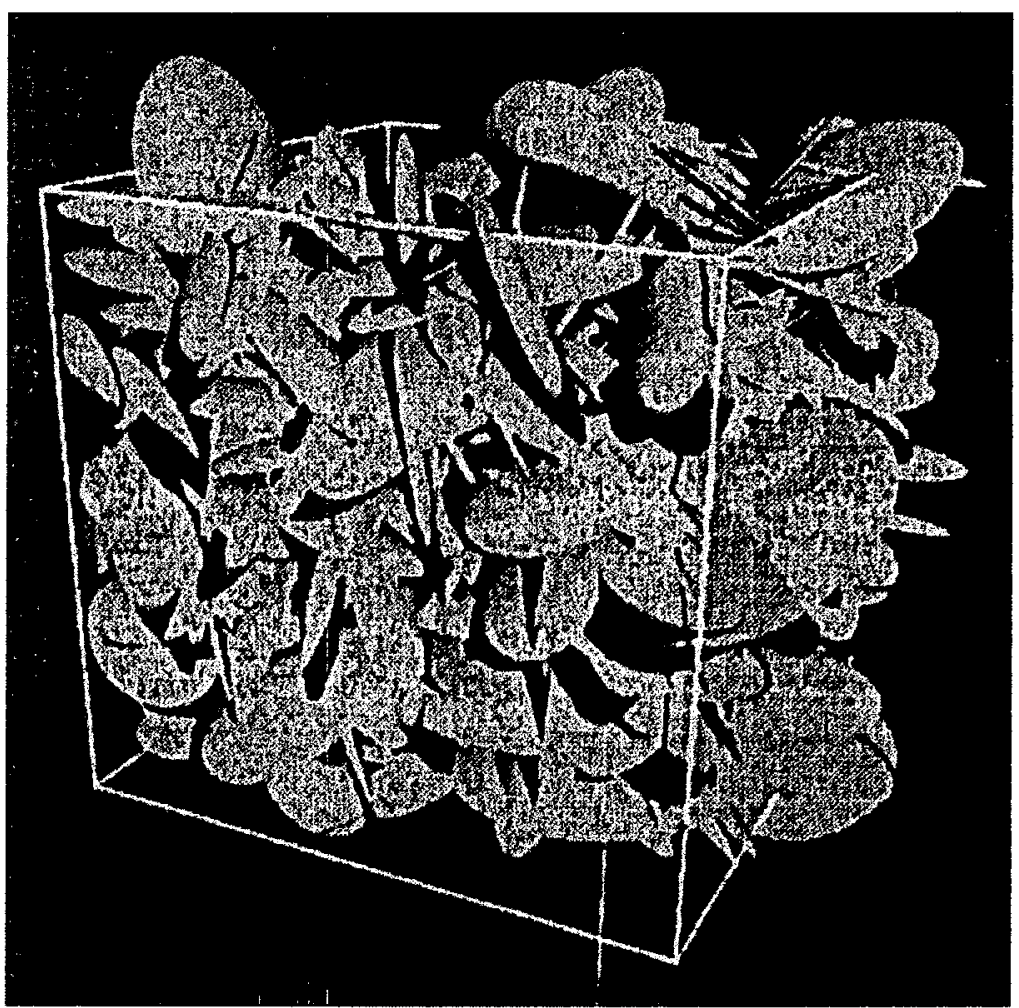

Figure 12: Generated 3D fracture network (Jing, 1998)

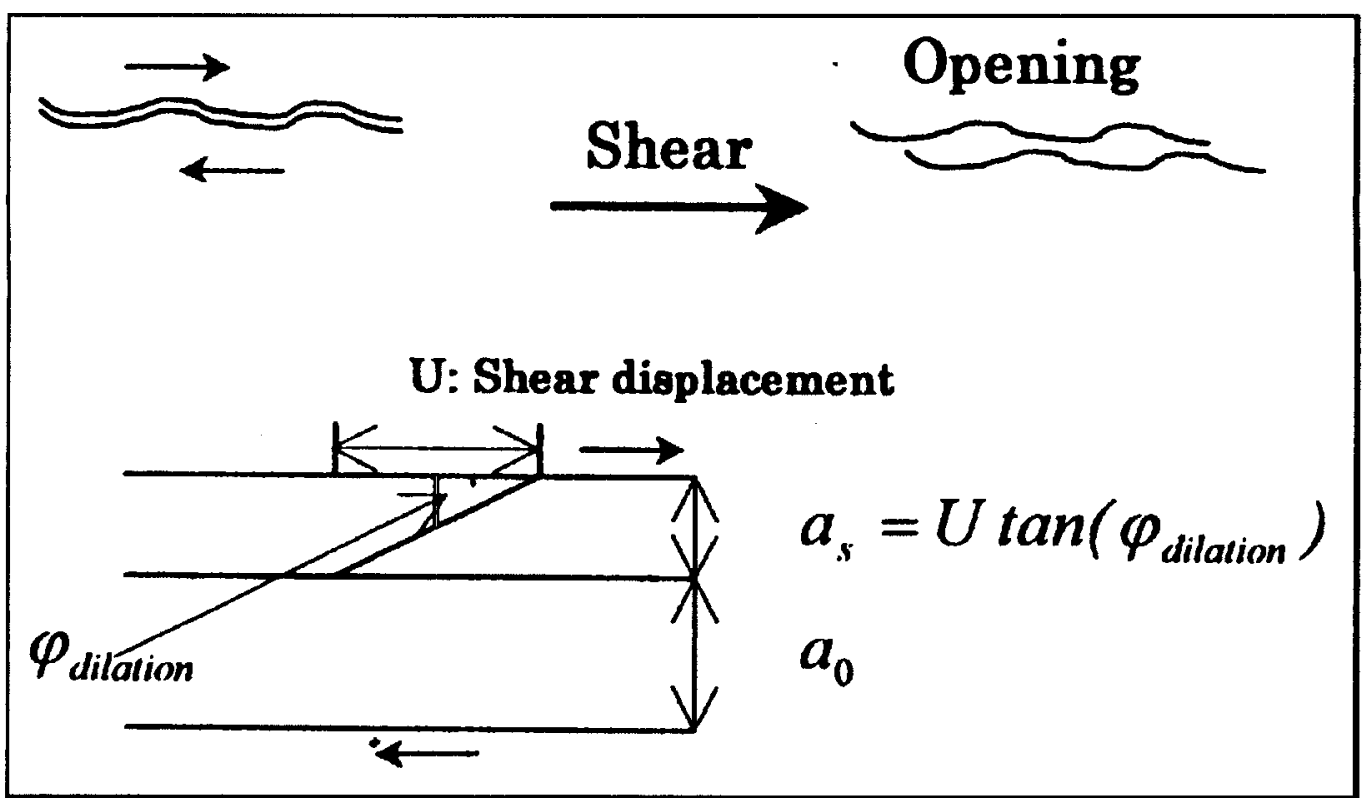

Figure 13: Relationship of fracture opening to shear displacement (Jing, 1998) 

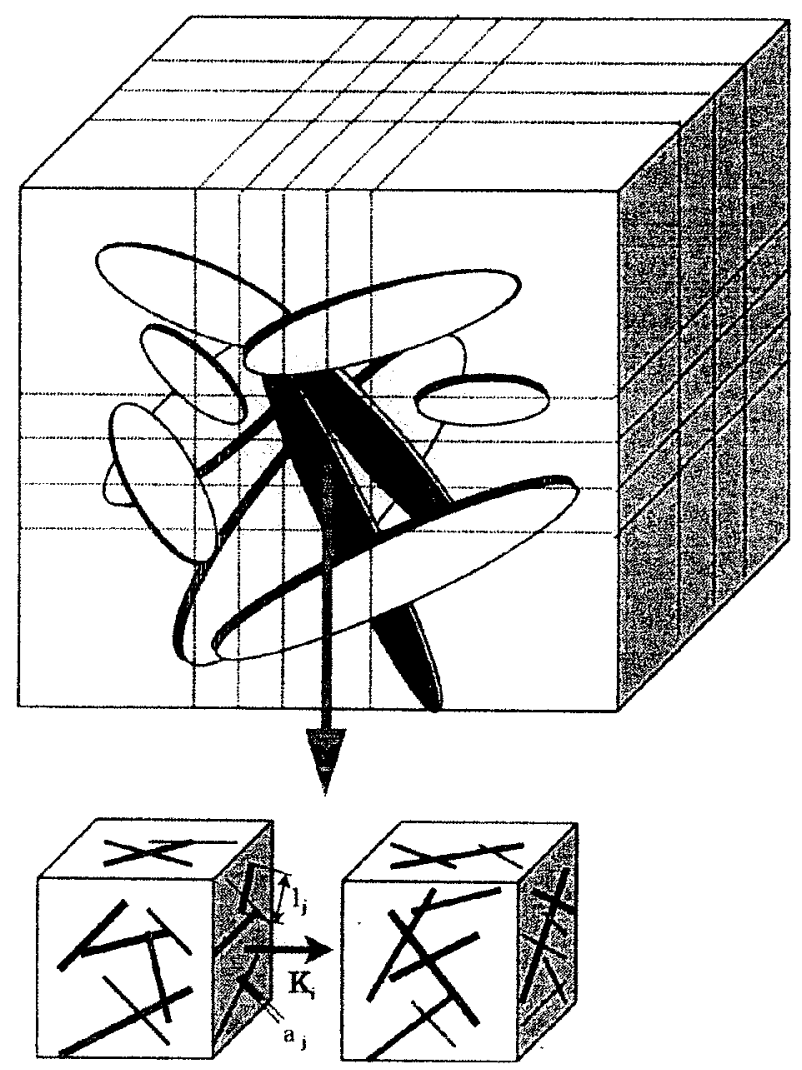

Figure 14: Calculation of equivalent permeability (Jing, 1998) 

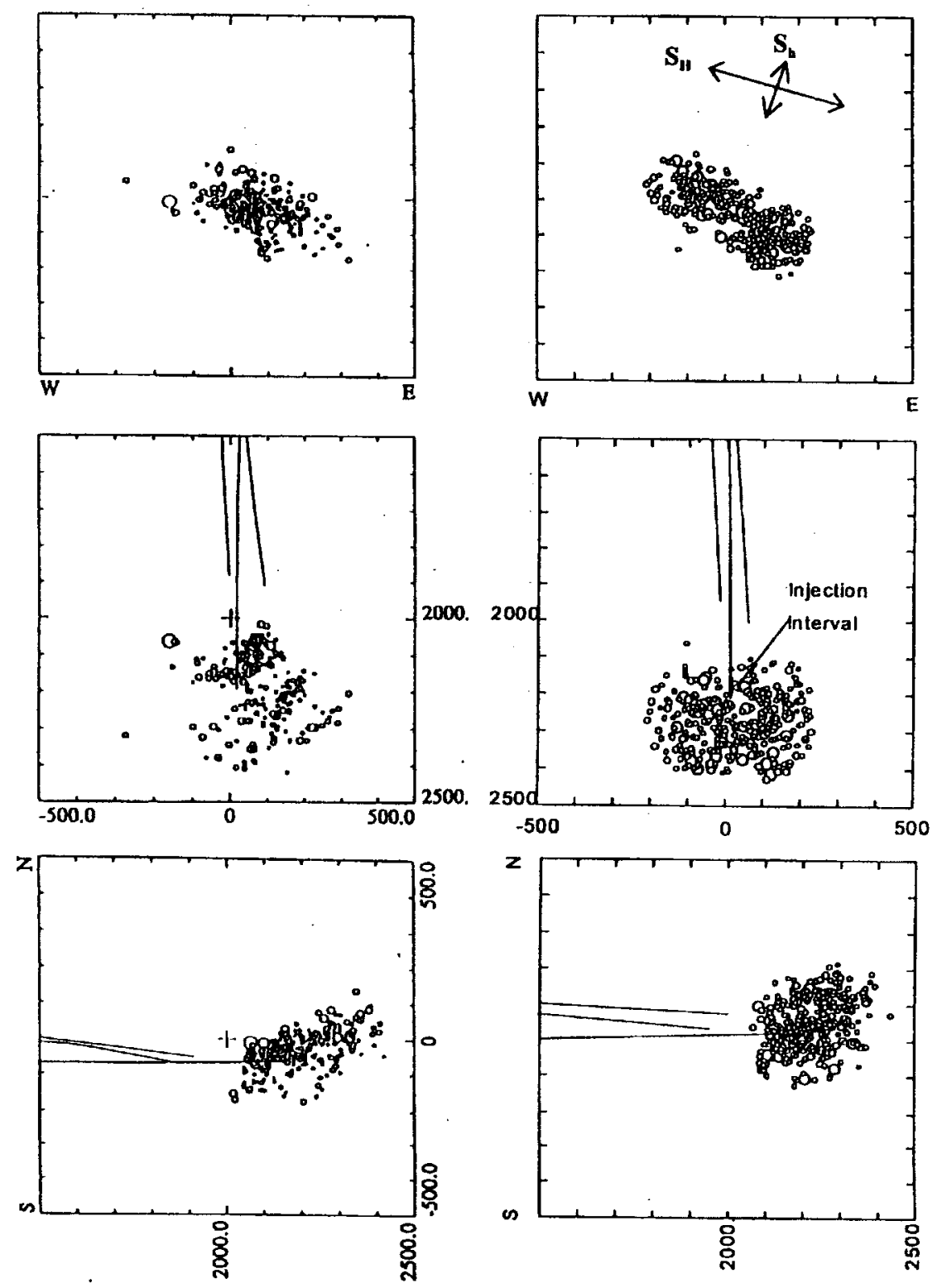

Figure 15: Comparison of measured acoustic emission pattern (left) and predicted stimulation volume (right) at Hijiori reservoir (Jing, 1998) 


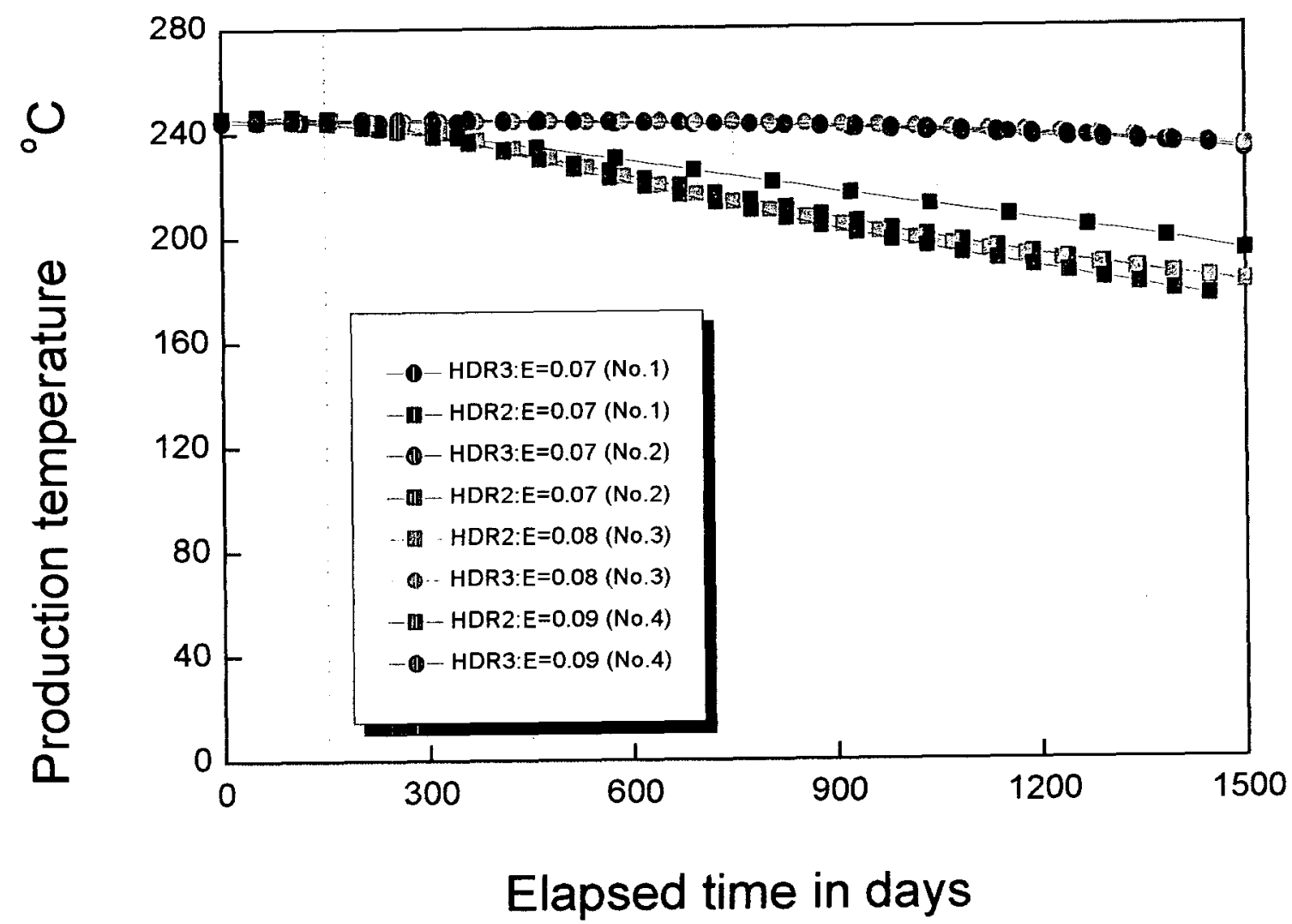

Figure 16: Long-term thermal drawdown (Jing et al., 1998) 


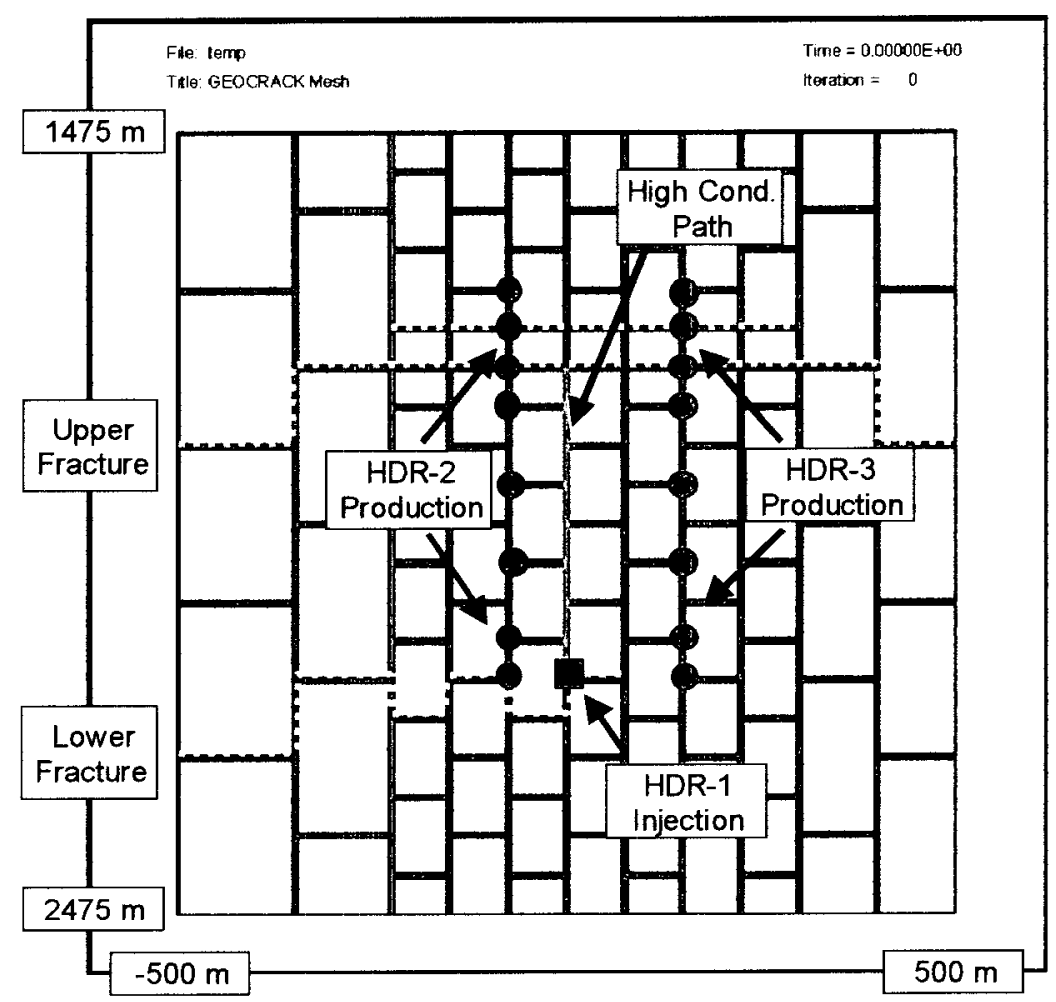

Figure 17: Geocrack2D model of Hijiori reservoir (Swenson et al., 1999) 


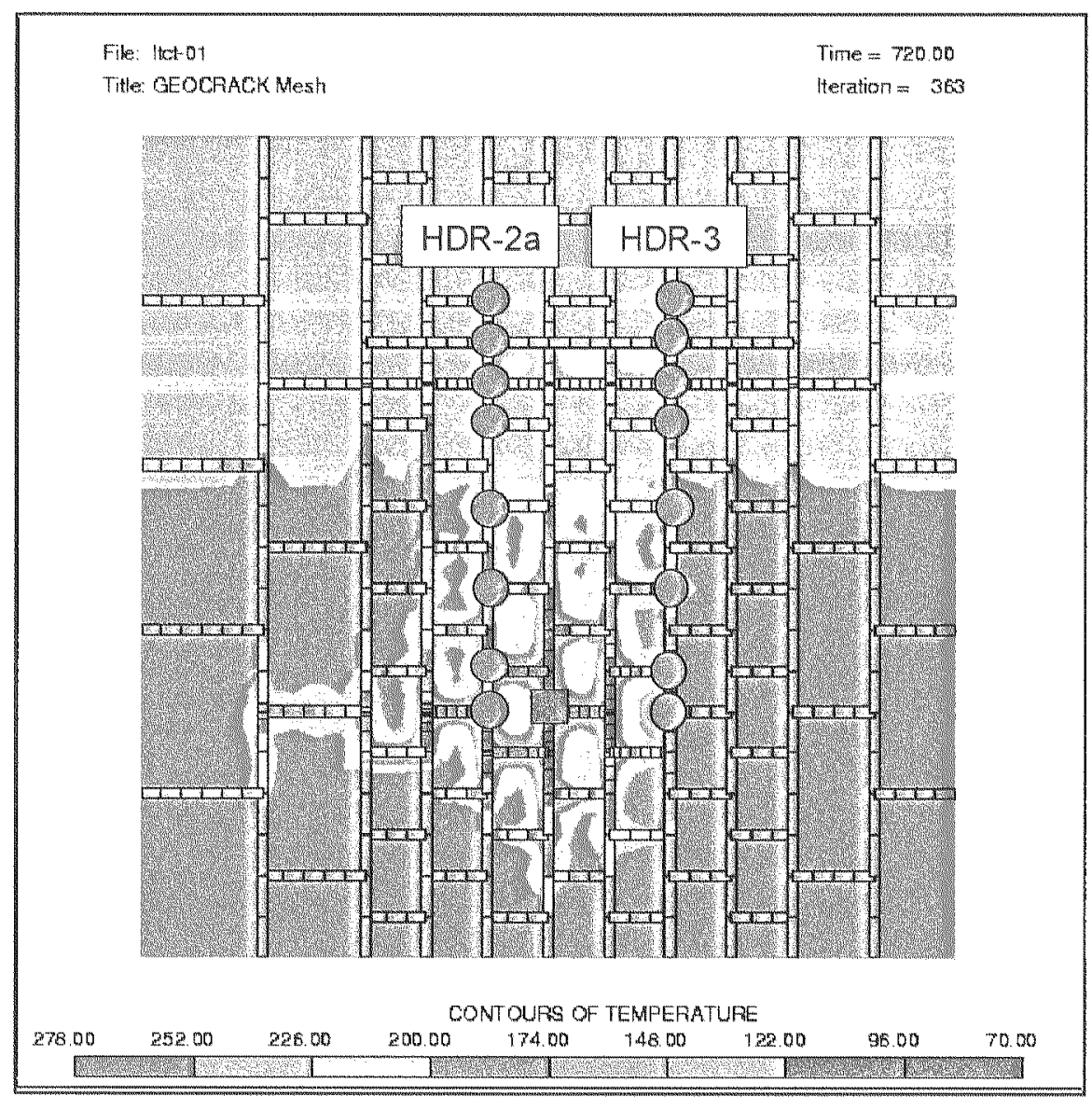

Figure 18: Temperatures in reservoir after 720 days of operation

$$
\left(\min =70^{\circ} \mathrm{C}, \max =278^{\circ} \mathrm{C}\right)(\text { Swenson et al., 1999) }
$$




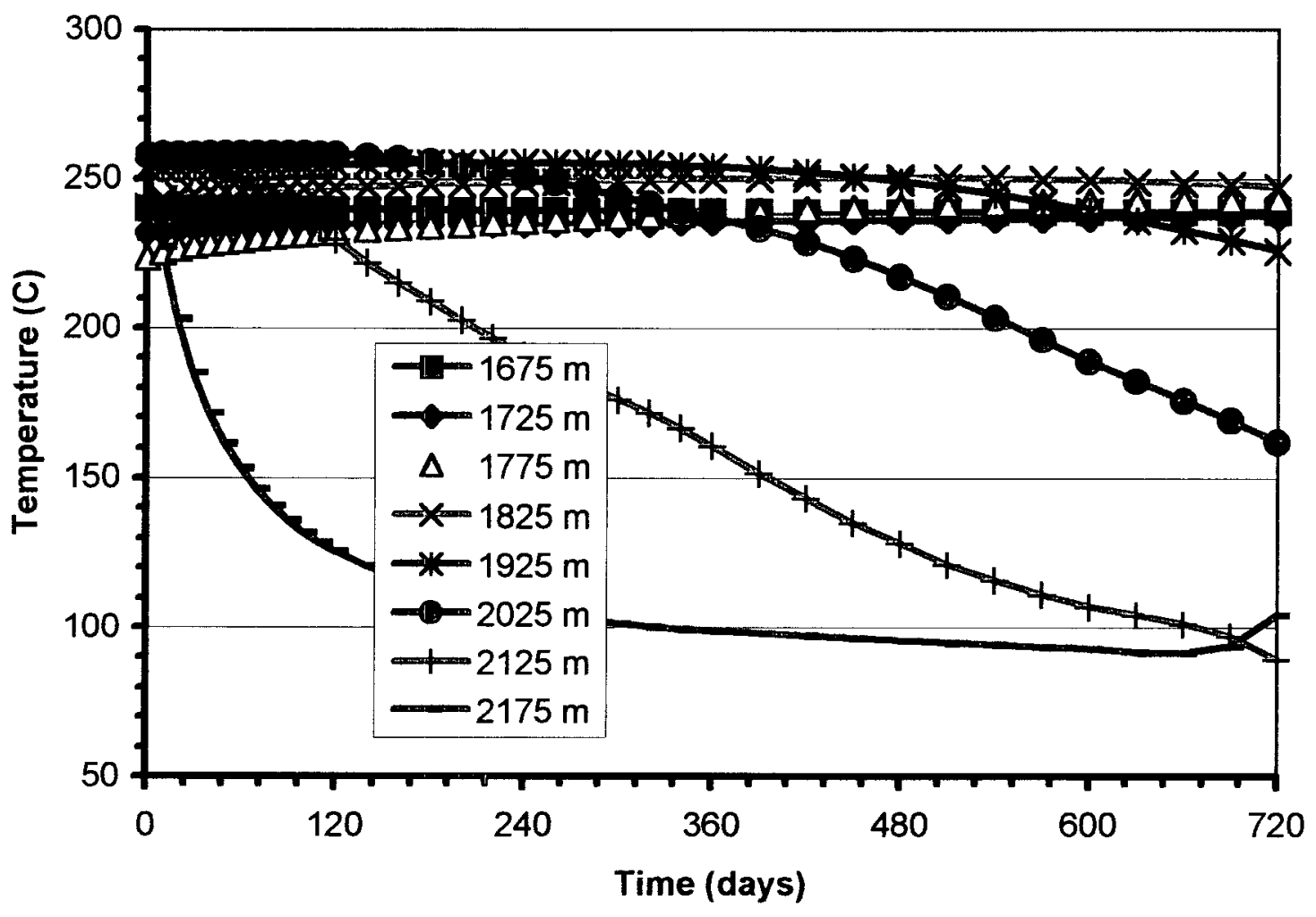

Figure 19: Calculated LTCT production temperatures from HDR-2a (Swenson et al., 1999) 


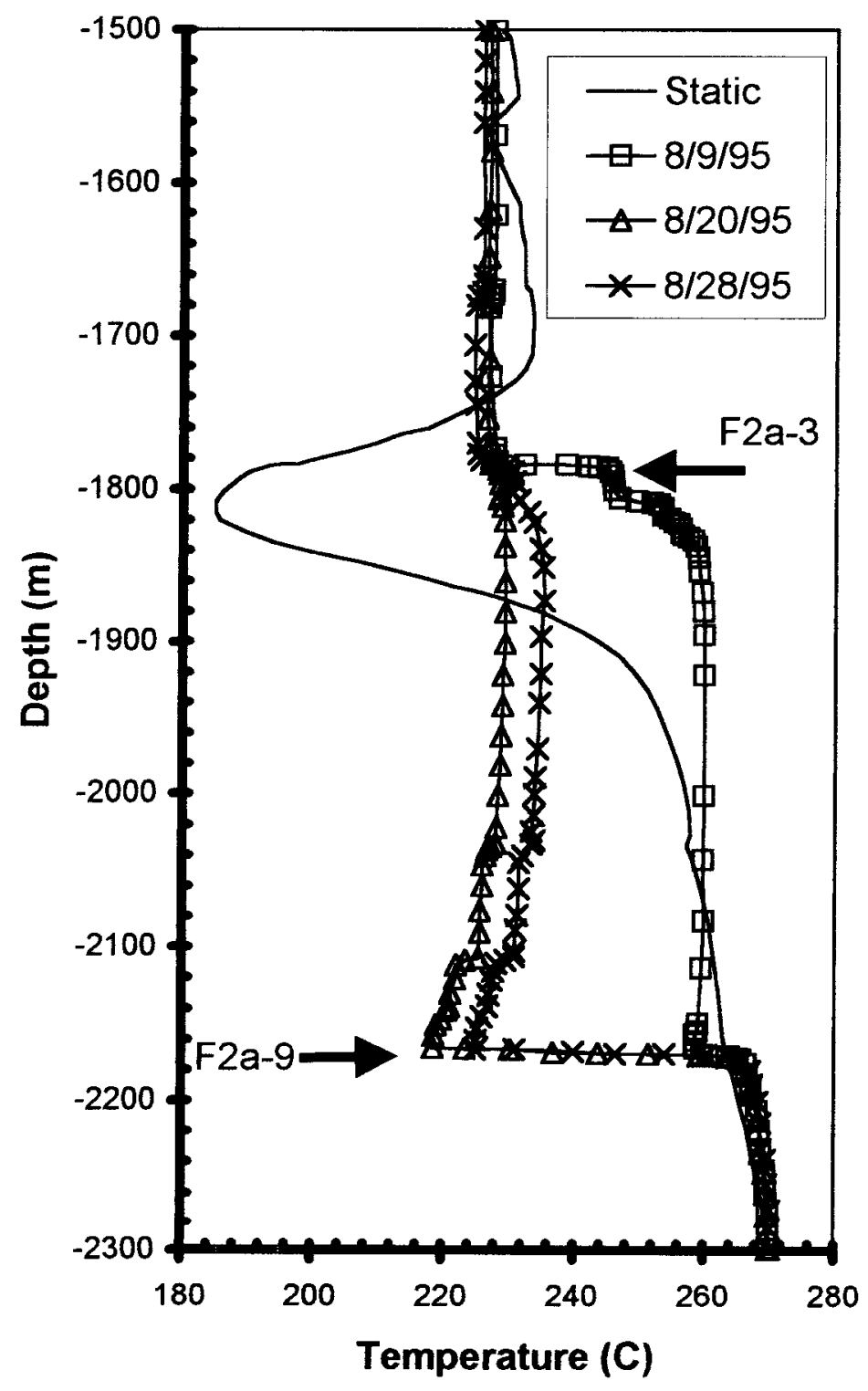

Figure 20: HDR-2a 1995 PTS data showing cooling at lower fracture (Swenson et al., 1999) 


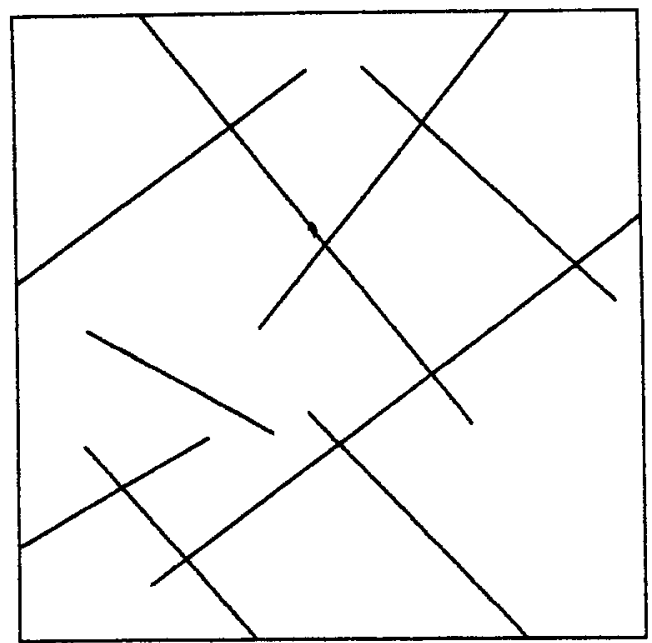

a.) Fractures

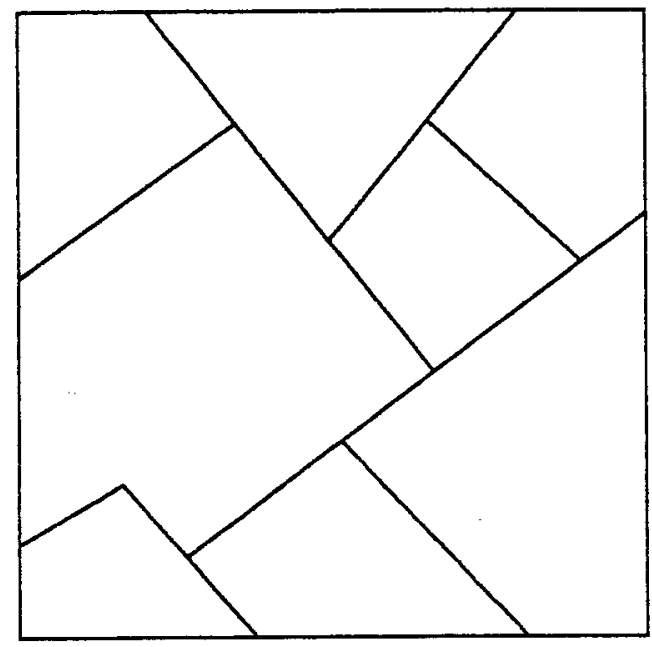

b.) Connected fractures

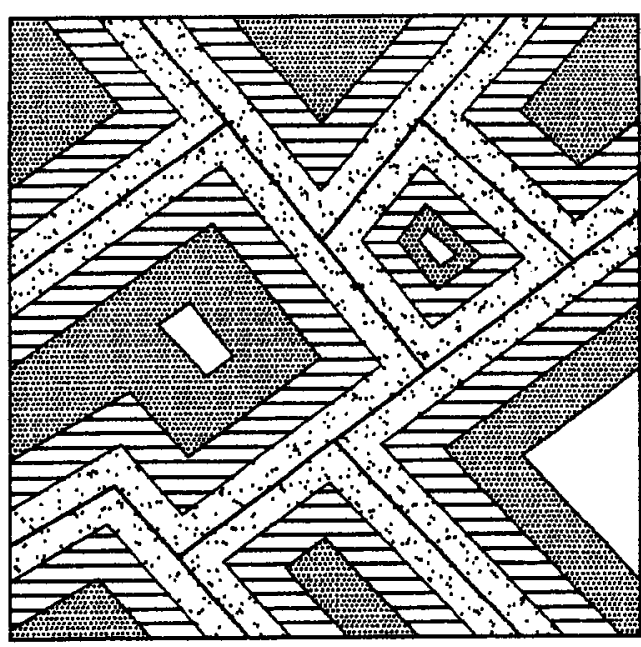

c.) MINC partitioning

Figure 21: Schematic representation of MINC partitioning using TOUGH 2 


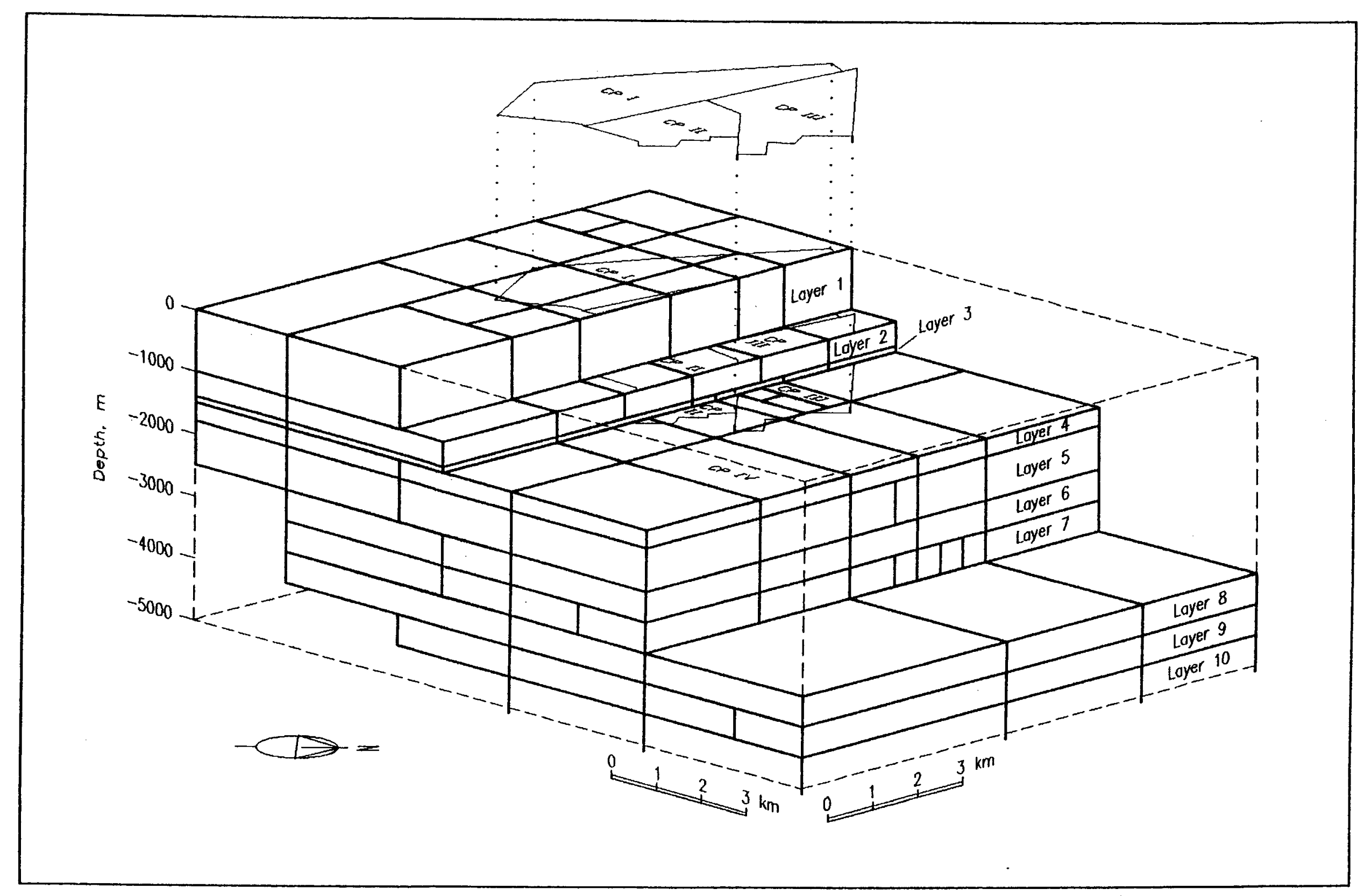

Figure 22: Three-dimensional view of the simulation model for Cerro Prieto. 


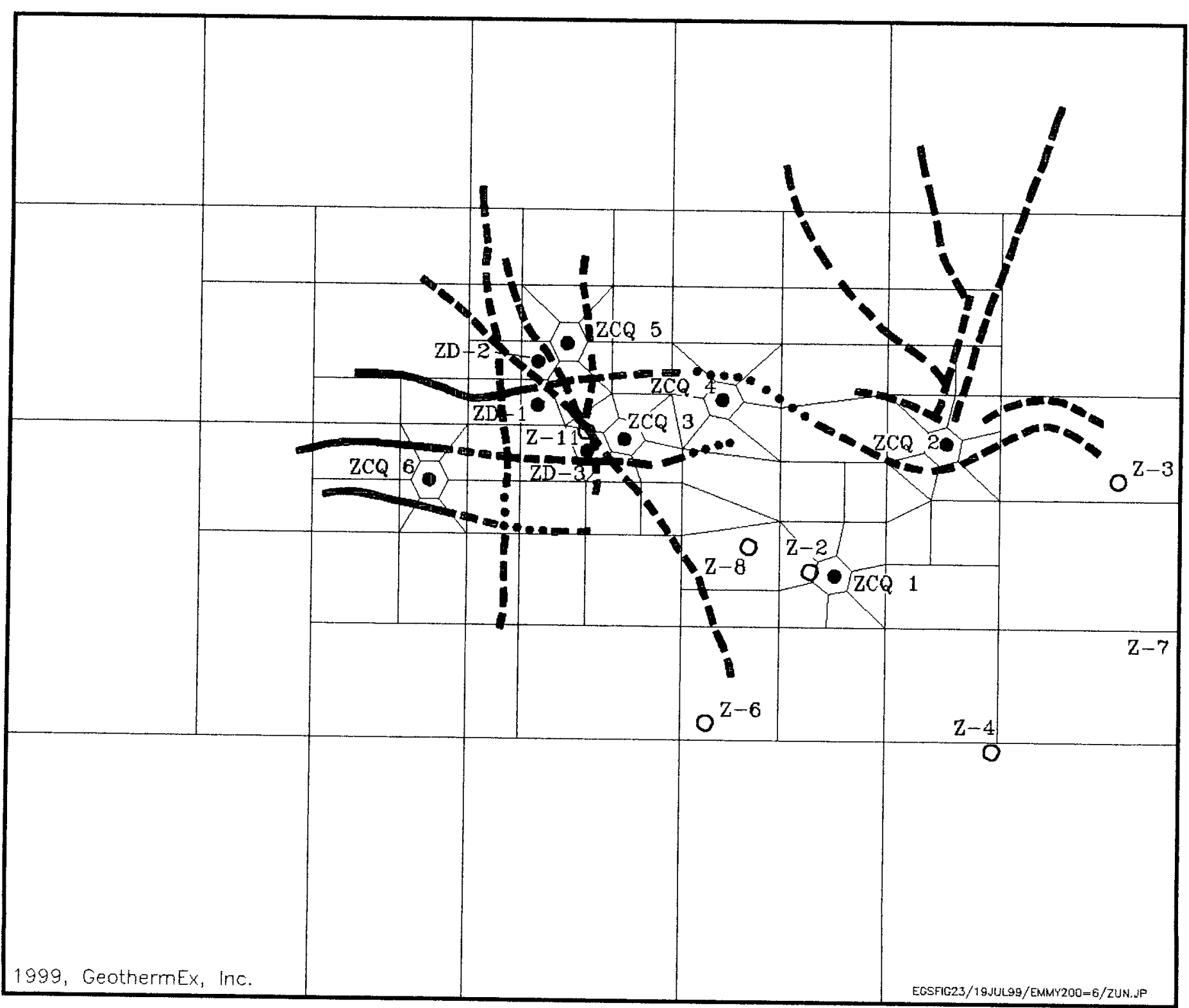

Figure 23: Example of modeling with an irregular grid 

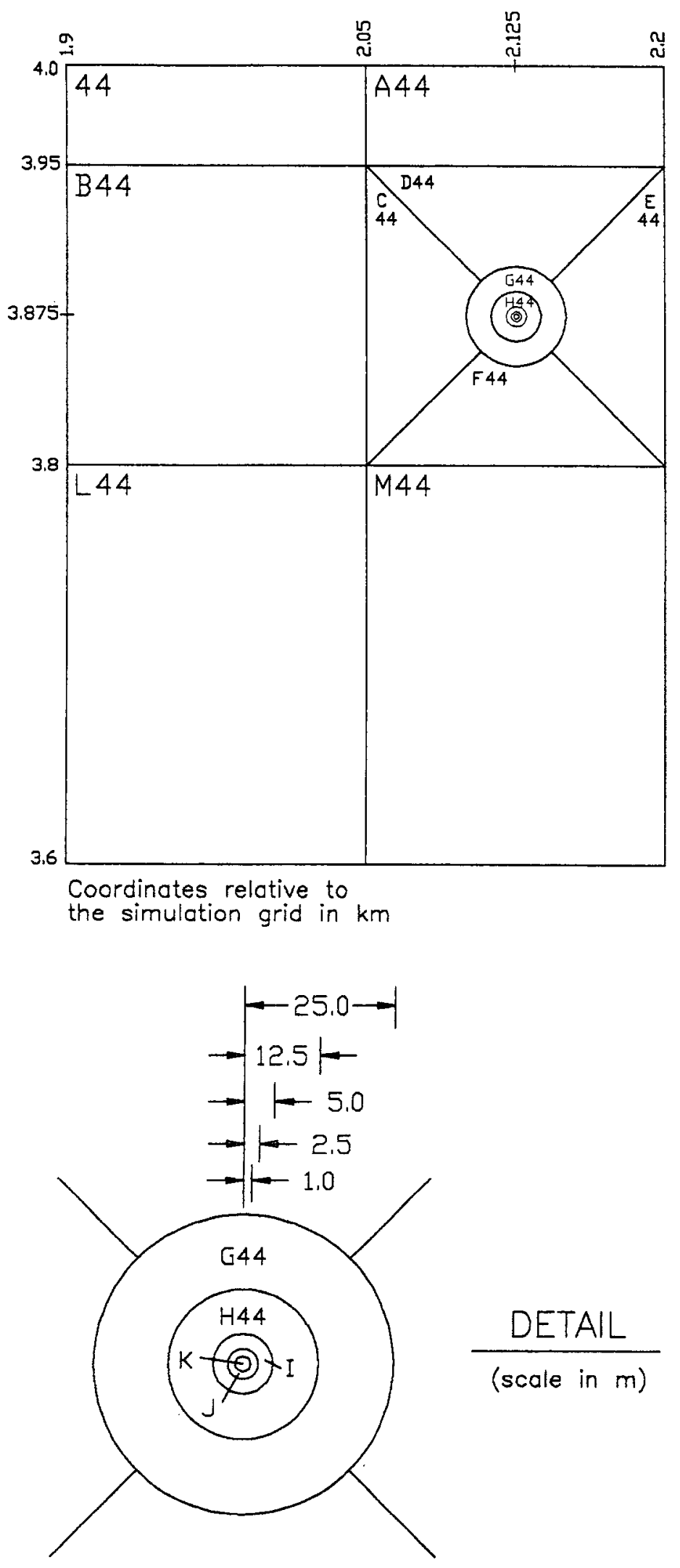

Figure 24:

An example of grid block refinement using TOUGH 


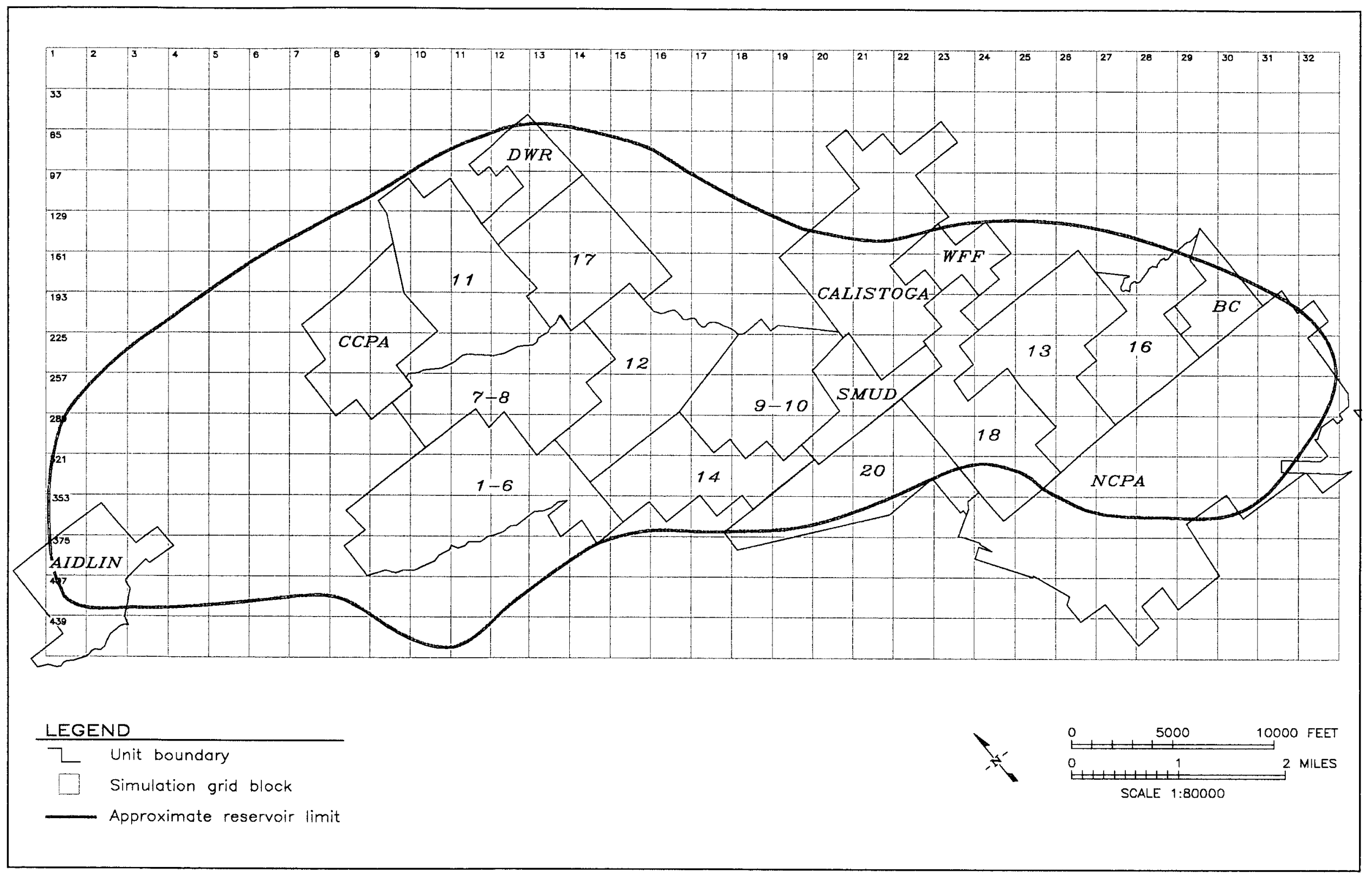

Figure 25: Grid layout for a numerical model of The Geysers geothermal field 


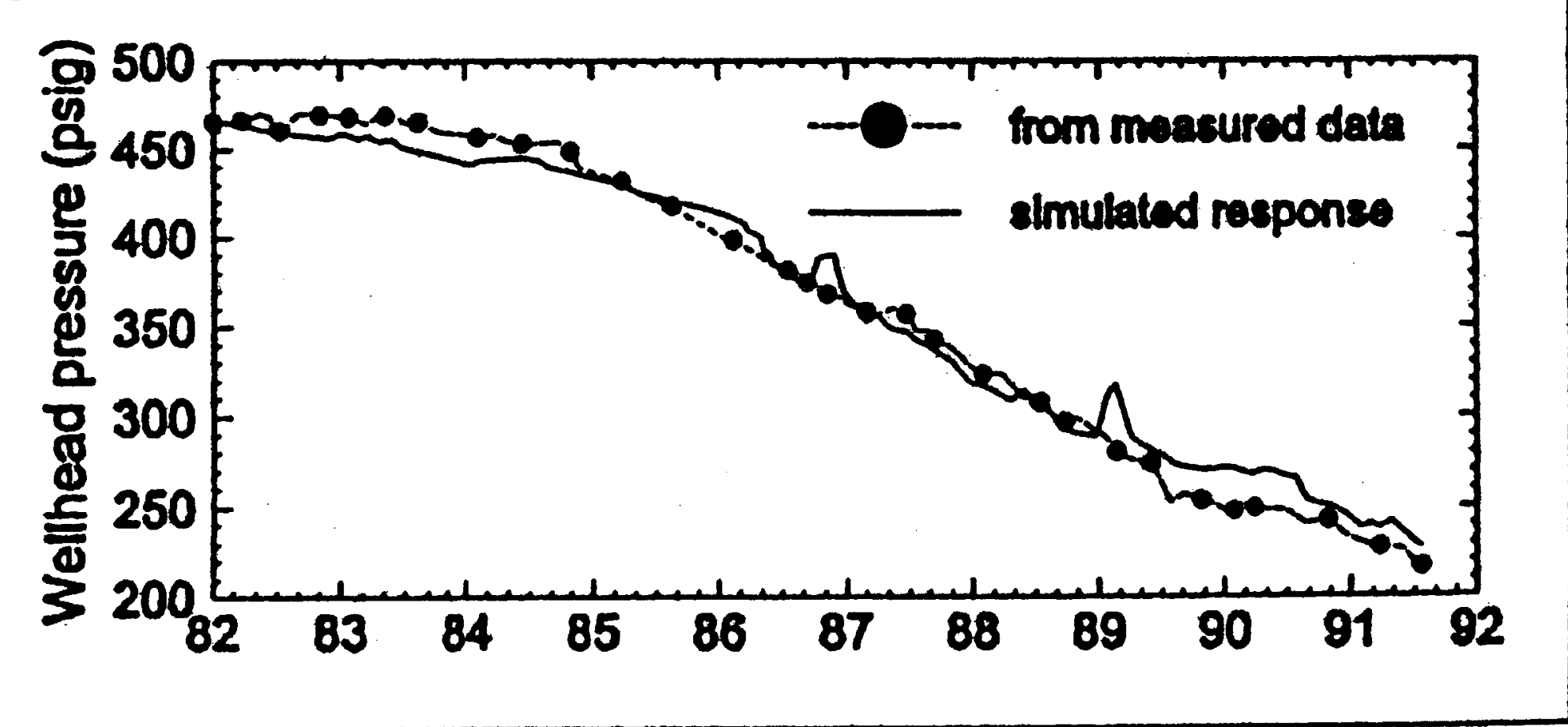

Figure 26: Example of pressure history matching

GeothermEx, Inc. 
Figure 27. Initial-State model of the Beowawe hydrothermal field, developed using TETRAD

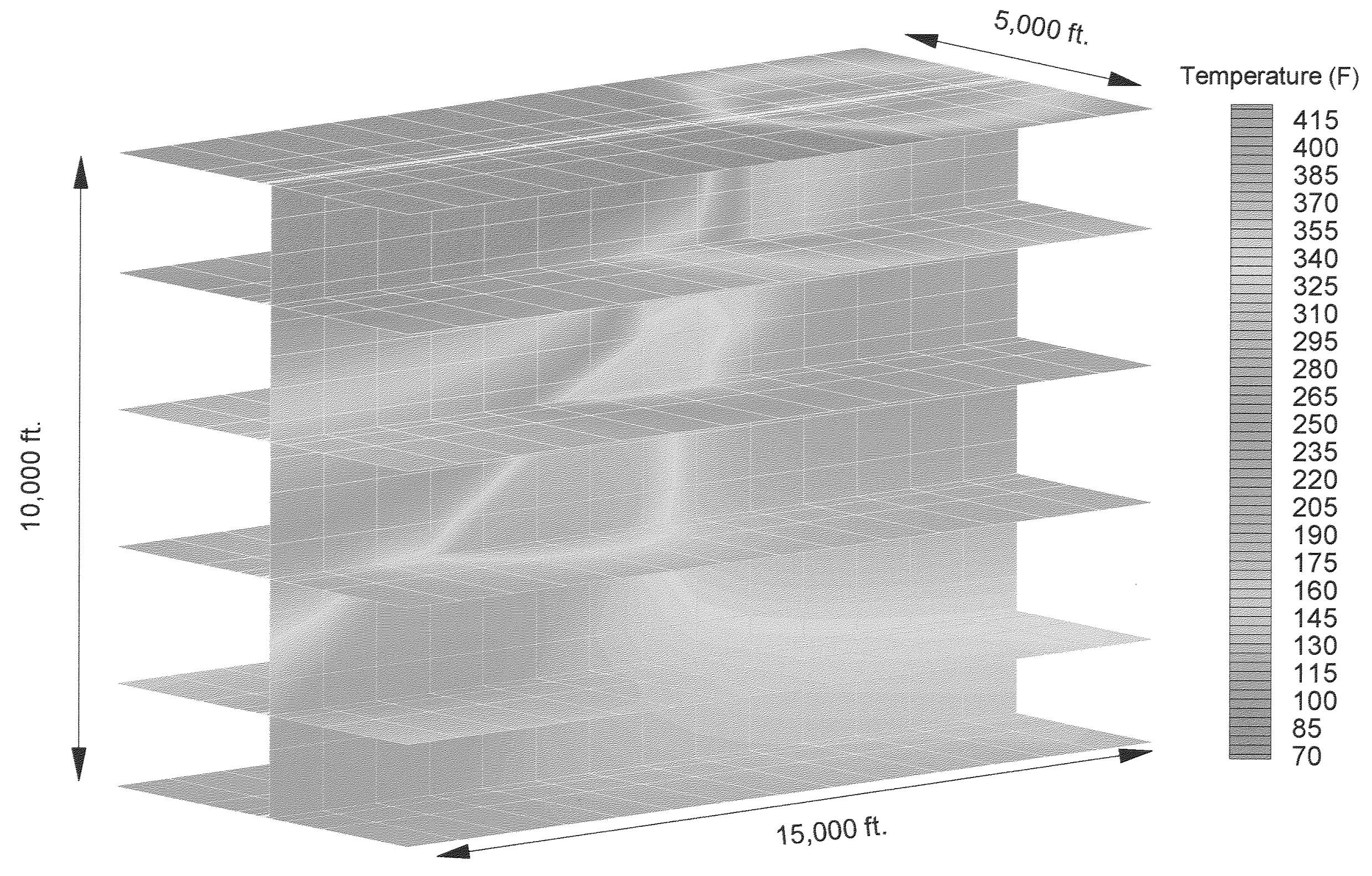




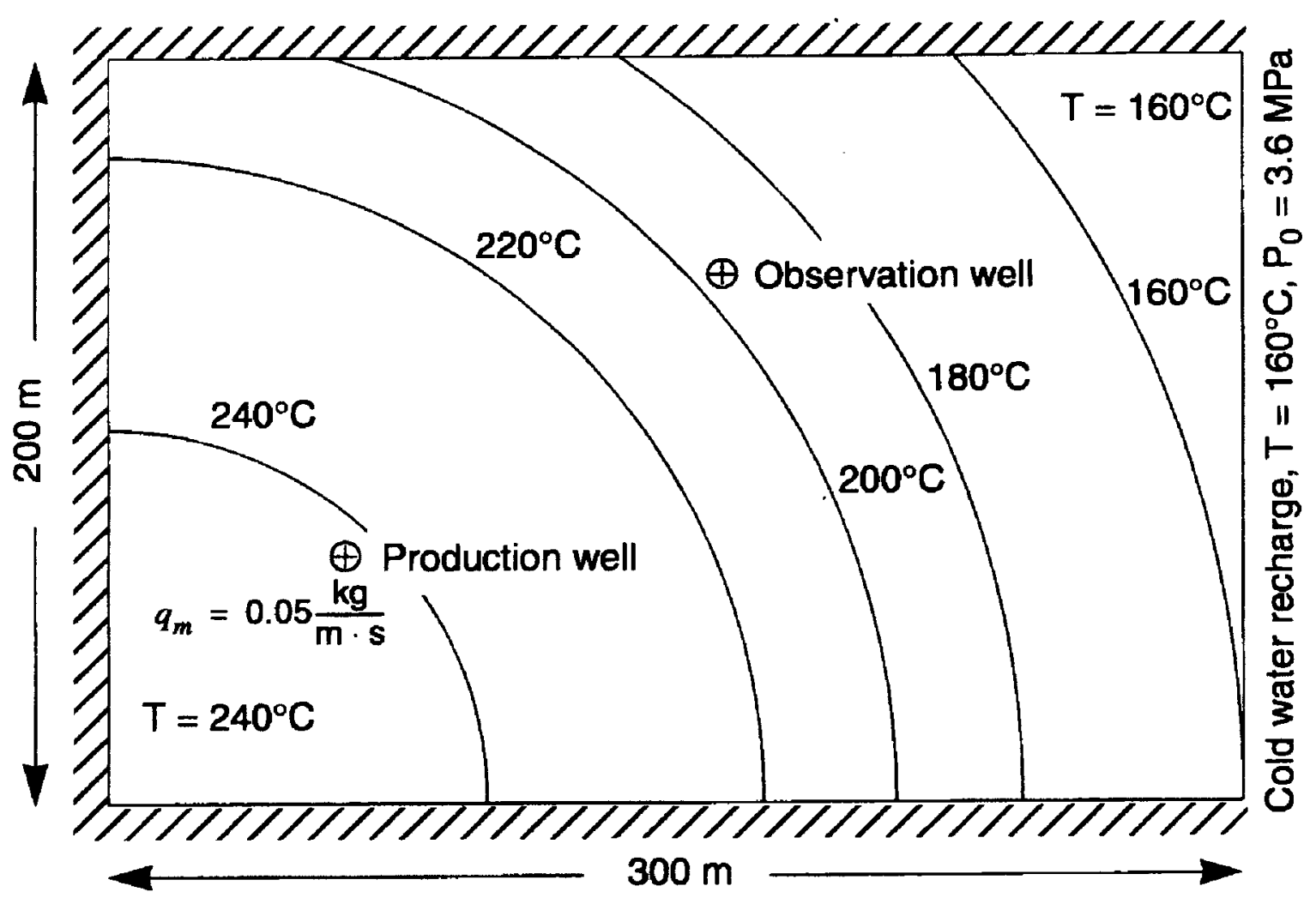

Figure 28: Problem definition for FEHM example (Zyvoloski et al., 1997) 


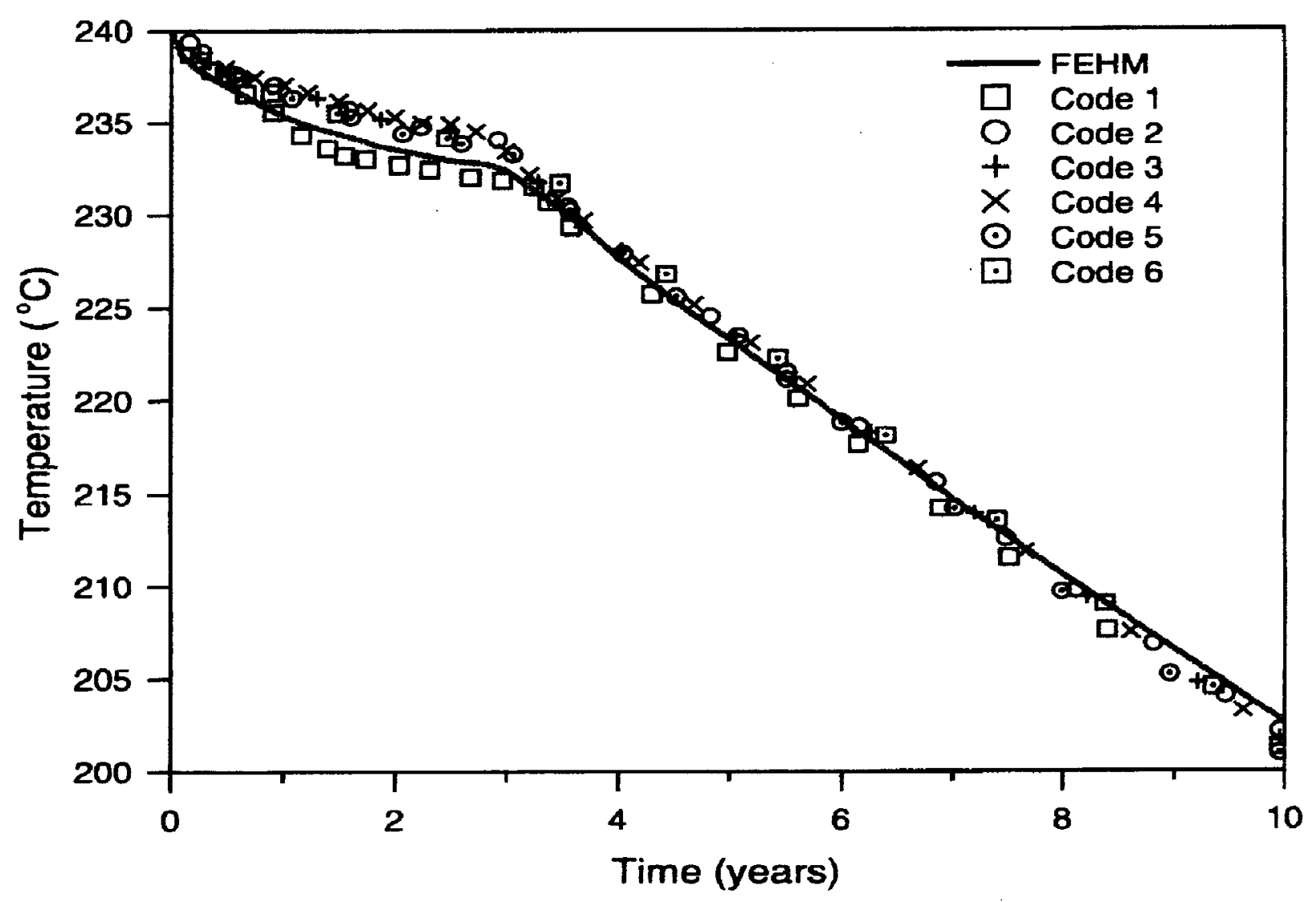

Figure 29: Comparison of FEHM production well temperatures with other solutions (Zyvoloski et al., 1997) 


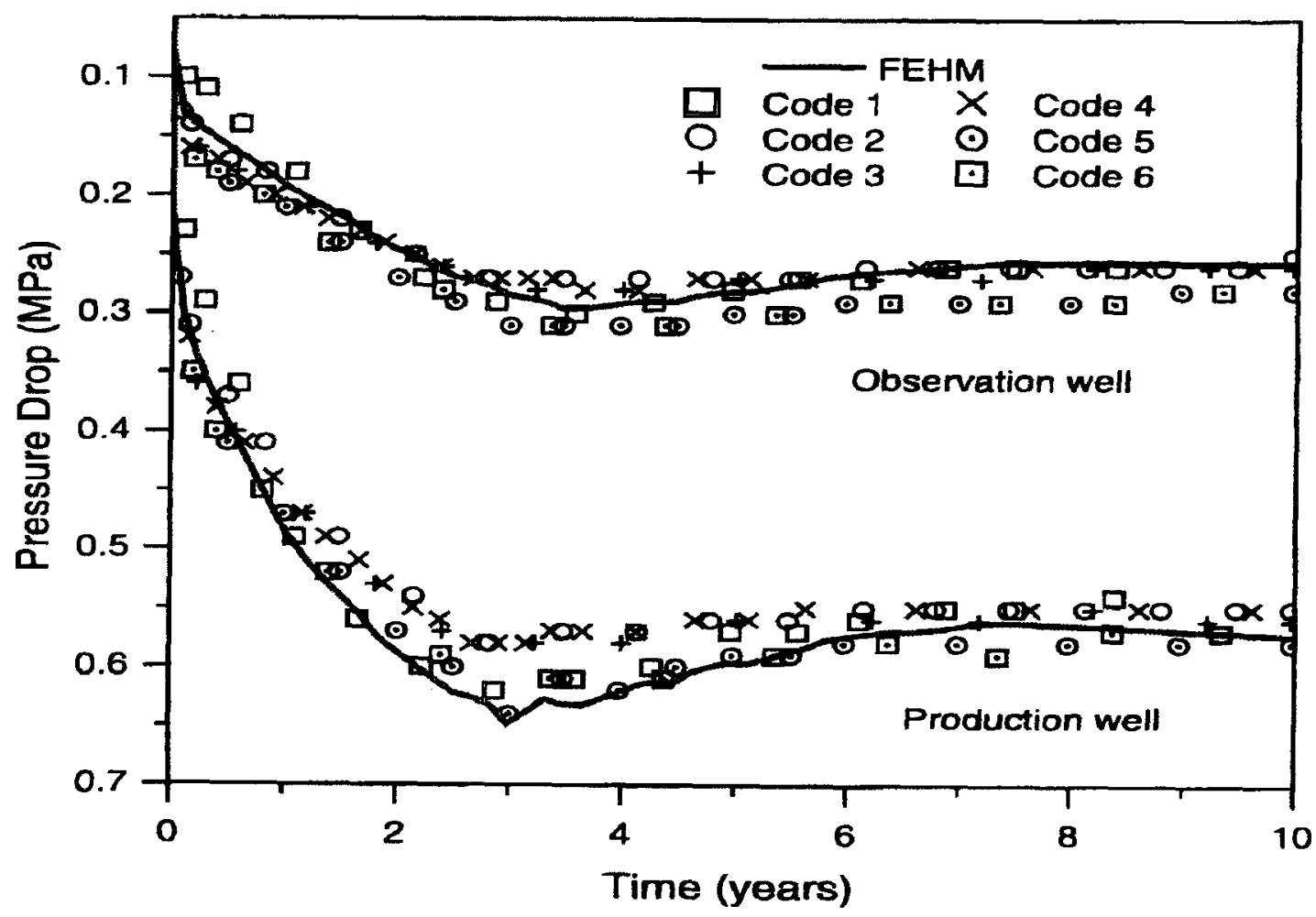

Figure 30: Comparison of FEHM production and observation well pressure drops with other solutions (Zyvoloski et al., 1997) 
E-MAIL: MW@GEOTHERMEX.COM 


\section{CONTRACTUAL INFORMATION}

This report was undertaken by GeothermEx, Inc., and two sub-contractors (Thunderhead Engineering Consultants and Golder Associates), under Task Order DE-AT07-98ID60317 of Contract DE-AM07-97ID13517. Princeton Energy Resources International (formerly Princeton Economic Research, Inc.) is the prime contractor for this contract, and GeothermEx is a subcontractor.

The original Task Order and the modification thereof are included in the following pages. The original scope of work called for a test problem to be devised and run by the various developers of the HDR and hydrothermal codes, and the results compared. This was Task 4, which is incorrectly stated on the original Task Order (the description of Task 2 is repeated erroneously). However, because of: 1) difficulties in getting commitments from several of the code developers to run the test problem;2) the unexpected addition of a fourth hydrothermal simulator (STAR) for review; and 3) the ability to review both public and private nuclear waste isolation codes (instead of only publicly available codes as originally anticipated), we requested a change in scope on 27 April 1999. This was approved on 5 July 1999 as indicated in the revised Task Order. 


\section{U.S. DEPARTMENT OF ENERGY TASK ORDER}

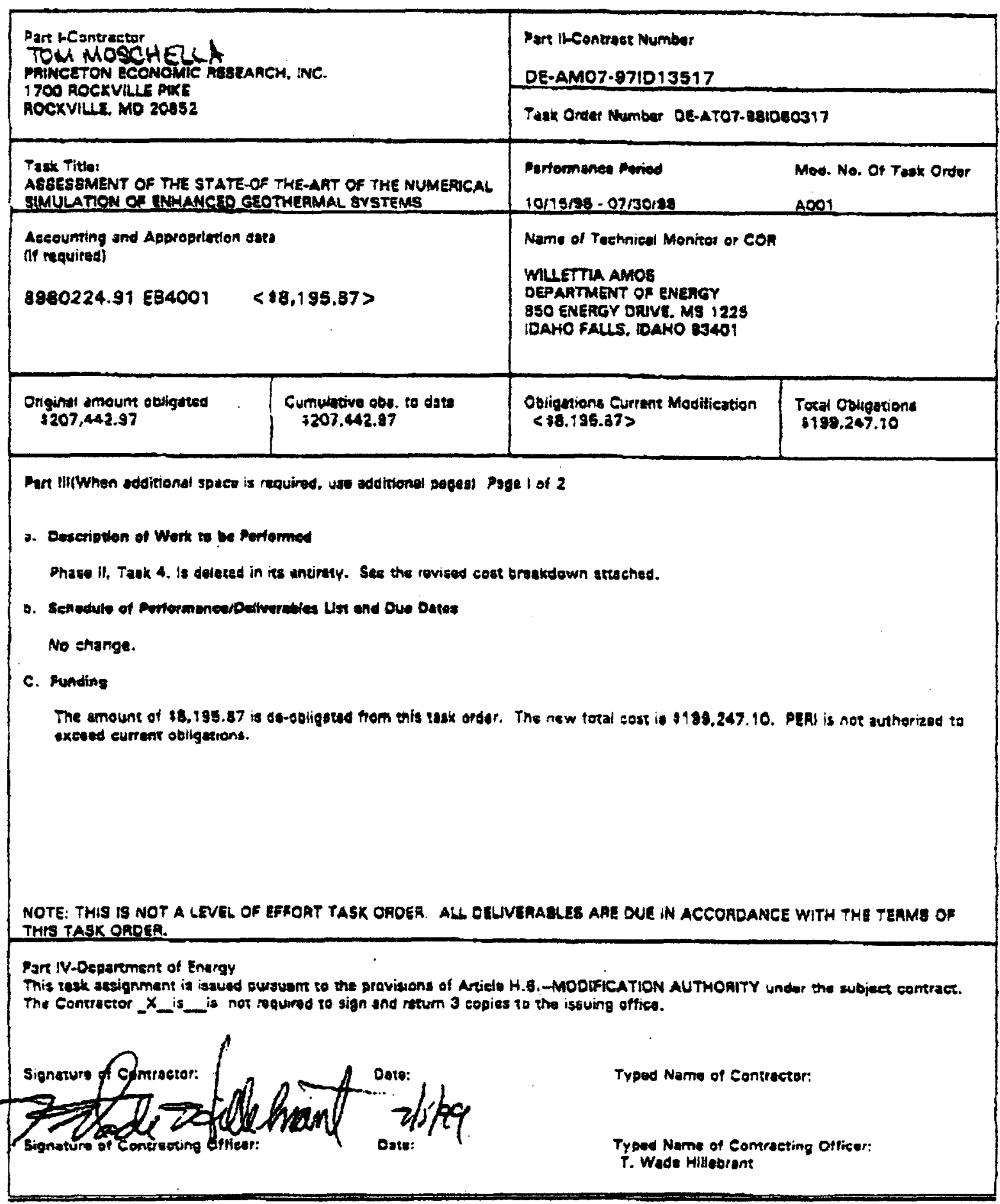




\section{STATEMENT OF OBJECTIYES}

Assessment of the State-of-the-Art of the Numerical Simulation of Enhanced Geothermal Systems

\section{Statement of Work}

In addition to the tasks detailed in the Statement of Work under DOE Contract DE-AMO7971013517, the PERI/GeothermEx team (P/GX) will evaluate the state-of-the-art of Enhanced Geothermal Systems (EGS) modeling and potential directions to improve the assessment of EGS by numerical simulation. The effort will be led by GeothermEx, with support from Dr. Dan Swenson of Kansas State University, and Dr. William Dershowitz and Mr. lan Miller of Golder Associates. PERI will provide review of the draft and inal reports prepared by GeothermEx. This work will be done in wo phases.

Phase 1: Task 1

Task 2

Task 3 Thase II: rask 4

rask 5 ask 6 ask 7
Conduct a literature search to identify ithe existing reservoir simulation technology for hydrothermal and hot, dr! systems, and interview experts who are now (or have been) involved in numerical modeling of hot, dry systems, including those in the U.S., Japan, Europe, and Australia.

Determine the utility and limitations of each of the hydrothermal and hot, dry system simulators developed to date; particularly, in regards to their handling of fracture definition and fluid flow and heat transfer in artificial fractures.

Prepare an interim report documenting the results of Tasks 1 and 2.

Determine the utility and limitations of each of the nydrothermal and hot, dry system simulators developed to date, particularly in regards to their handling of fracture definition and fluid flow and heat iransfer in artificial fractures.

Determine if there are any publicly availacle simulators developed for contaminant transport or nuclear waste isolation, which would have application to simulation of enhanced geothermal systems. Possible areas of application include accurate geometric representation of fracture networks, geometric representation of in-plane fracture hetercgeneity, and analogs for heat transfer in muiti-rate matrix diffusion.

Recommend specific enhancements to existing simulators and/or development of new simulators which would enable better prediction of the performance of and would apply to the spectrum of enhanced geothermal systems.

Prepare a complete report documenting the results of Tasks 1 through 6 . The draft report will be reviewed by DOE. PERI, and industry before the final report is completed and submitted to DOE. 
E-MAIL: MW@GEOTHERMEX.COM

\section{APPENDIX B: Comments on the Draft Report}


TELEPHONE: $(510)$ 527-9876

FAX: $(510) 527-8164$

E-MAIL: MW@GEOTHERMEX.COM

\section{COMMENTS FROM DOE ON THE DRAFT REVIEW OF EGS SIMULATORS}

DOE reviewer(s) provided useful comments on the draft report that prompted significant improvements. The reviewer(s) comments are included below (in italics) together with our responses to them.

1. A reorganization of the document is recommended. There is no need for anything other than the tables and explanatory material. The benchmark for this type of effort is the EPA Compilation of Groundwater Models (EPA/600/R-93/118, May, 1993).

Unfortunately, the EPA "benchmark" report format was not specified in the statement of work, and the effort required to re-format the report exceeds the scope. Our report is comprised of tables and explanatory material, with some figures included to illustrate certain points. To set the stage for the code reviews, the text includes some background material that the authors felt would be useful for the reader. Inclusion of only the tables and explanatory material would greatly reduce the usefulness of the report to the reader.

The title is not correct. This is not a "state of the art assessment". There have been significant advances in estimating fracture geometry changes with stress. The implication of current research with respect to this report is that planar representation of existing and generated fractures is inadequate. Fracture generation codes must have the capability to generate "steered" or curved fracture planes with reasonable accuracy. This idea is critical to the proper evaluation of codes that may be used for designing and characterizing EGS stimulation.

The statement of work called for a review of available numerical simulators that could be potentially applied to EGS problems. Although non-planar fractures may occur in EGS and other reservoirs, none of the available simulators have non-planar fracture generators; hence, a 
TELEPHONE: (510) 527-9876

FAX: (510) 527-8164

E-MAIL: MW@ GEOTHERMEX.COM

discussion of such fractures is not included in the report. As regards the title of the document, it is correct according to both the work reported therein and the statement of work.

It is also disconcerting that the report mentions "proposed work" as if the report is laying the groundwork for future work.

As some background material for the introduction was taken from the proposal, the phrase "the goal of the proposed work is ... ." appeared inadvertently. We have corrected the sentence to read "the goal of this study is ...." There was no intention on the part of the authors to imply that further work should be undertaken. In fact, this study did not propose any specific further work.

As discussed below, the report is heavily weighted towards the authors' work and examples.

This is true. Some simulators are described in more detail than others because the authors have worked with some simulators more than others. We were given this job by DOE because our team is highly experienced in numerical simulation of conventional geothermal systems, HDR systems and nuclear waste isolation problems. Our work and examples are the natural result of our experience. We brought up certain examples to illustrate the features of the simulators; this is discussed further below. With regards to the simulators used to model hydrothermal systems, since very few well-documented case histories of such simulation of geothermal systems exist, the authors had little choice but to rely on some of their own experience.

The criteria spelled out in Section 2 (p 2-11) are presumably the ones that simulator features must be compared against. Some of these requirements are relatively simple to implement in existing simulators (e.g., a relationship between fracture aperture and conductivity) assuming a valid mathematical model is available. This distinction might be important in determining which 
TELEPHONE: $(510)$ 527-9876

FAX: (510) 527-8164

E-MAIL:MW@GEOTHERMEX.COM

codes are most readily adapted for EGS systems. Note that the development of such models does not appear to be within the scope of this effort.

As the reviewer(s) suggest, the development of such models was not within the scope of this effort. The report points out to what extent these features are included in the various simulators reviewed, and a discussion of implementing the various features has been added in Section 7 .

What might be appropriate is to list and prioritize the required simulator features, and then the desirable features. Prioritization might be done on the basis of utility or ease of implementation (with the caveat noted above), or some weighted function of both. This list could then be used to "weed out" various of the codes currently discussed. For example, any code that does not satisfy the minimum criteria might be listed, but should not be reviewed as being too far away for practical interest; instead a single paragraph might be included that notes the code does meet criteria $1-3$ and 5-6, but not ...

Of course, care would have to be taken in developing the criteria. For example, none of the available HDR-codes currently have multi-phase flow. For hydrothermal codes, that might be a showstopper; for an HDR-code, it might be merely ranked high on the desirable features list. A code that does not consider energy balance might be too far away from use so as to be impractical. A different set of criteria for each class of simulator might be desirable.

One of the conclusions of this work was that although no single simulator has every feature, not every feature is needed in a given problem. For example, the ability to handle mineral deposition and dissolution is needed in some simulation problems, but not all. Therefore, prioritizing the list of simulator features would be somewhat misleading, and we may do a disservice to some of the simulators in the process. Effective development and use of simulation codes require a level of experience (and indeed the intuition developed from it) to determine what features are needed for a given simulation effort. For example, it is useful in some cases to include non-condensable 
gases in a numerical model. Does this mean that every simulator should be able to handle gases? We think not. Even if a field has gas and a simulator is chosen that can handle gas as a component, it may not be necessary or desirable to include gas in the simulation effort. Perhaps the power plant has adequate gas handling capacity for any anticipated gas increase, or no increase is expected. The point is that prioritizing simulators on the basis of whether or not they have certain potentially desirable features could easily give a false impression of the relative usefulness of a given simulator.

2. An executive summary would be helpful that summarizes the recommendations. For most users of this document, reading through code descriptions is more effort than is likely will be expended.

We totally concur on this point. Although the Recommendations section (Chapter 8) is three pages long and could be the focus for someone who does not want to read through the code descriptions, we have added an Executive Summary in response to this comment.

3. All codes being reviewed should be subjected to the same criteria. For example, the Table of Contents should have the same entries for each code, at least within a given simulator type. Where additional features exist (e.g., geochemistry), those should be spelled out in a final section for that code, perhaps entitled "Other Features."

Using the same criteria to review each simulator would indeed be desirable. However, all simulators do not have the same features and abilities, which precludes this uniform approach. One example of this is the ability to handle rock deformation (i.e., elastic behavior). By looking at the Table of Contents, the reader can see if a simulator has that feature or not, and can focus their review of the report accordingly. 
TELEPHONE: $(510) 527-9876$

FAX: (510) 527-8164

E-MAIL: MW @GEOTHERMEX.COM

4. It appears that the simulators being reviewed are not done so even-handedly. Examples abound, and will be pointed out below as well. Governing equations are discussed in some depth for some codes (e.g., Geocrack, FEHM) and not at all for others (e.g., TETRAD, STAR). In other cases, governing equations are mentioned (SWIFT), but the references are to standard mass and energy balance equations, not specific to the equations in the code discussed.

This comment is quite valid; the review is not even-handed. We solicited information from the developers of each code, and could only present what was provided, augmented in some cases by our own knowledge. Sometimes the developers provided the specific equations that are used in the code, but in other cases the available information referred to standard equations for mass and energy balances, etc. To get the governing equations of all the codes would require us to purchase each simulator and get the manuals, which is clearly beyond the scope of this report. The authors' familiarity with the different codes varies significantly. To clarify this, we have added an explanation in the introduction why different material is presented for each code. We have also included information about two of the authors being developers of two of the codes reviewed (Daniel Swenson for Geocrack2D and Golder Associates for FracMan/MAFIC) and about GeothermEx's extensive experience with TOUGH2 and TETRAD.

The same problem exists with the discussions of applicability. In some cases extensive example problem discussion exists; in others none. A good example is the treatment of STAR. Despite having been validated against the Stanford Geothermal problem set (1980), no example solution is presented. Other codes (FEHM) use one of those very problems to show typical application. TETRAD is highlighted in 2 examples.

Again, we appreciate this comment, and offer a similar response. Examples were provided by some code developers and not by others. For Geocrack2D, TOUGH2 and TETRAD, we used examples from our own experience because they were available. The inclusion or exclusion of examples or equations is not meant to imply any favoritism for one simulator or the other. It is 
TELEPHONE: $(510)$ 527-9876

FAX: (510) 527-8164

E-MAIL:MW@GEOTHERMEX.COM

simply a result of what was available to us. We point out that STAR was not used for the Stanford Geothermal problem set, but rather an earlier simulator developed by S-Cubed. We do not know of a case history of a simulation using STAR, nor was one provided by the authors of the code. The FEHM code was indeed validated against the Stanford geothermal problem set; therefore, these details are provided for this code.

5. Listing governing equations for the codes should either be done in detail for each code, summarized as a general set of conservation equations, or removed from the document entirely. It is not appropriate to discuss equations for some models and not others. It gives the entire document the appearance of bias towards one or more of the models (e.g., some 3 pages devoted to discussion of Geocrack governing equations). It might also help to avoid criticism of the report if somewhere in it mention is made of the fact that one of the reports' contributors is also the author of Geocrack.

See comments replying to the apparent lack of even-handed reviewing above. We have added some text in the introduction that describes the method of obtaining information about the simulators and the authors' involvement in their development and use. We believe that removing all equations from the report to remove any perceived "bias" towards any particular simulator would drastically reduce the usefulness of the document. Reducing everything down to the lowest common denominator would provide substantially less information to the reader, and would therefore make the report less valuable.

The same observation applies to discussions of features that will appear in future releases of a given code (e.g., Geocrack3D, TOUGH2 v. 2.0). One cannot know whether these statements are completely true, given uncertainties in code $V \& V$, or when such new versions might be available to the general public. The impression given is that all other codes are stagnant, whereas these select few continue to be dynamic. That is likely not true, but may reflect one of two conditions. First, that input on new features was only received from authors of these codes (solicited from 
TELEPHONE: $(510)$ 527-9876

FAX: (510) 527-8164

E-MAIL:MW@GEOTHERMEX.COM

all?), or that the report authors have better information on these select few. The result is the same; the report appears biased towards 1-3 of the codes discussed.

This is a very valid point and the final version of the report focuses on the version of the code that is available today, with only passing remarks in the text about features under development. To clarify, however, we point out that we mentioned features that were under development because such information was available to us, not because of any bias.

It seems more useful to discuss code capabilities in more general terms (rather than detailed equations). One might assume that a model whose governing equations purport to conserving mass (for example) do so, and let it go at that. Additional or novel governing equations (e.g., geomechanical models) can be referred to without giving the equations ("...the integrodifferential form of the conservation equation is used (Pruess and Narasimhan, 19??), or "... a modified Biot equation is used to ..."). Either that, or go over all governing equations for all models (or at least the ones that satisfy minimum criteria).

See above starting with the response to reviewer(s) comment \#4; they apply here as well.

6. Several problems exist with regards to the Recommendations Section. First, the authors' specific recommendations address only the need to improve the "HDR type" simulators, not the hydrothermal ones. I would be tempted to conclude from this that the HDR simulators either have more of the required features, or that they will be more easily modified to accommodate requirements. If this is so, it seems that would be an important observation. If not so, perhaps you should be more even in recommending funding/improvements.

We have made several clarifications in the Recommendations section. Hydrothermal simulators handle most of the features (some in an approximate way) needed for EGS simulation. However, the HDR-type simulators are not yet useful for practical simulation of EGS projects 
adjacent to existing hydrothermal developments, which is the direction the EGS program is headed in the near term. In such projects, the focus will be on the effect of an adjacent EGS project on the behavior of the entire field. Considering that there is likely to be a long production history, many existing wells, and two-phase flow in the reservoir, a robust simulator that can handle these types of conditions is needed. It is our opinion that such simulators, despite their lack of, for example, discrete fracture networks and dynamic fracture characteristics, can be used to accurately predict the impact of EGS development, while HDR simulators cannot. The obvious conclusions are: 1) lack of the "perfect" simulator is not an impediment to EGS development adjacent to an existing hydrothermal development; and 2) HDR-type simulators are the ones that need improving.

The 4th of the authors' recommendations has nothing whatsoever to do with the stated intent of the report (or the authors scope), and should be removed entirely from the report. It may be a true statement, but has no place in this document.

We have removed it as suggested. The purpose of making this statement (about the need for research to identify and define the hydraulically active fractures in a reservoir) was to emphasize that the simulation is only as good as the information that goes into it. This is of course a more fundamental problem than others addressed in this report. Although fracture definition is not part of the scope of the report, it is important to consider when evaluating: 1) where R\&D money should go; and 2) the accuracy and utility of a fracture-based numerical model.

\section{Several terms are used in the document that warrant better definition. For example, some} codes treat irregular grids. What specifically is meant by irregular? Is a curvilinear grid considered regular? I assume what you mean is a grid that can explicitly treat non-orthogonal fractures. If that is the case, state it. 
In common usage, an "irregular grid" implies a non-orthogonal grid. We have added explanatory text in the final report to clarify this.

Likewise your discussion of geochemistry. What geochemistry is thought to be required? All of the hydrothermal models treat non-condensible gases, all or nearly all treat the effects of $\mathrm{NaCl}$ on phase behavior. If the need exists for reactive transport, state that more explicitly, or add another footnote: one that indicates equilibrium geochemistry, one that hands non-equilibrium.

We agree that geochemistry should be defined. The geochemical processes of interest in EGS modeling are primarily those related to mineral deposition and dissolution and the subsequent effect on fracture permeability. We have modified the text and tables to clarify this.

\section{Comments of Table 1, 2.}

Use of some of the footnotes is confusing. On Table I FRACTure is noted to have laminar and turbulent flow equations. Does this mean it also has flow rate as function of aperture (the question being posed in the table)? That is not stated; if it is true, state that and add the footnote is necessary. Likewise footnote 5: possible w/ user-defined material properties. That sounds like the answer is yes, but in the text it is stated that channeling probably could be done. My reaction is that channeling affects the transport of chemical species (which perhaps could be done with a suitable dispersivity) and a reduction in surface area for heat transfer to occur. If the authors' opinion is that this could be done within FRACTure, it might be worth a bit more of a write-up (to defend that statement, particularly with regard to decreased surface area), and then a YES in the Table.

We agree that the footnotes are confusing and have clarified them in the final report. 
Use of Footnote 6 is confusing. Possible means that it can be done. That implies the answer is YES. If it can't be done as yet (pending a new version, or something), the answer is NO.

Given the fact that nearly $1 / 2$ of the "positive" entries for Geocrack are either "possible" (see comment above), or "under development" rather than NO, the document is once again given the appearance of bias towards Geocrack. Given one or more of the authors' affiliation that is easy to understand, but difficult to refrain from commenting on. If the desire to document that code's capabilities outweighs the need for objectivity, perhaps this review report should be postponed until Geocrack $3 D$ is complete and distributed. If under development is an appropriate explanation, all code authors should be given the same opportunity to respond in a similar fashion.

We agree with all of the above comments and have modified the tables accordingly.

9. The following comments come from specific observations in the report.

p ii. All simulators in a given class should have identical subheadings and topics discussed.

See our response to the reviewer(s) comment \#1 above.

$p 2.8$, first paragraph of 2.8 indicates the limited willingness of researchers to incorporate chemical coupling in codes is in part due to uncertainty and inadequacy of mathematical models. That comment applies to many of the desired features discussed in Section 2. If appropriate (well-defined) math models were available, many features could be readily implemented in existing codes. Without useful models, however, implementation of new features merely provides still another dial that can be turned in order to obtain a match point.

We certainly agree with this point, and have added some text in Section 7 that discusses this. 
p 2.11 see comments above on reorganization of report. It would be useful to list these features (required and desired) in some prioritized list, and then only discuss codes that satisfy some fraction of those.

As mentioned above, the choice of simulator depends on the problem at hand. Therefore, we believe it would be misleading to prioritize simulator features.

p 2.11 another feature mentioned in the text but not listed is being coupled to a wellbore model. That should be included in the list of features, and discussed with regard to each of the models.

All of the hydrothermal simulators reviewed can be coupled to a wellbore model, while the HDR simulators cannot. However, since wellbore simulation is peripheral to (rather than a feature of) numerical simulators, we have removed the reference to it.

p 3.2 Equation 3.2.1 is not a statement of Darcy's law; it is a statement of conservation of mass.

This comment is valid; although Darcy's Law is used in the equation, it is not a statement of Darcy's Law per se. The text has been modified accordingly.

p 4.6 The text states that, as the most widely used simulator in the world, TOUGH is the most validated. This is a non-sequitor; because the code is more widely used does not imply that all such users have conducted validation exercises.

Validation exercises by various users (including GeothermEx) have consisted of successful matching of both the initial-state and the production/injection history, and forecasts of reservoir behavior that have been compared against the actual performance subsequent to forecasting. Judging from the number of case histories presented year after year at the TOUGH Workshop, 


\section{GeothermEx, Inc. ${ }^{2221}$ CENTTRAL AVENENE, SUTTE 201

TELEPHONE: $(510)$ 527-9876

FAX: (510) 527-8164

E-MAIL: MW@GEOTHERMEX.COM

one can hardly question the validation of TOUGH2. However, we have taken out the phrase ". . is most validated."

p 4.7 The text states that: "As in TOUGH 2, interaction ... by Warren and Root (1963)". This is true of TETRAD, but not of TOUGH unless the user specifies the appropriate grid lengths, etc in the MINC formulation. That is to say, a W\&R approach is not the standard approach in TOUGH.

We agree, and have changed the text accordingly.

p 4.8 TETRAD also has tracer options that allow radioactive and thermal decay, adsorption, etc.

The text has been modified to include this information.

p 4.11 The discussion of application of STAR is poor. One could even refer to the 1980 Stanford code comparison report and take the $S$-Cubed results from that. Failure to report any again shows a lack of even-handedness.

See our response to reviewer(s) comment \#4 above.

p 4.12 Section 4.5.3 refers to Fluid Flow, and gives the governing equation for heat conduction.

This is correct; the text has been modified accordingly.

p 4.13 Equation 4.5.3 is given as conservation of energy, but (assuming the nomenclature given is correct) I don't see any flux of energy. Shouldn't there be some heat capacities and temperatures in the flux terms? I still recommend that the governing equations be removed. 
The variable " $\mathrm{D}$ " in this equation is the transmissibility, including enthalpy as appropriate in the energy equation.

p 4.14 In the Coupling of the Models section: is this discussion of dual porosity/permeability thought to be specific to FEHM? It is not, but in either case, this discussion of Coupling of Models is inconsistent with other such discussions (c.f. p 3.17 on Geocrack).

We agree and have moved the text describing the various formulations of FEHM to Section 4.5.1.

p 5.2 The discussion of Hydraulic Behavior of FLAC is confusing. Is it true that flow is considered incompressible, but density changes are accounted for using the Ideal (not Perfect) Gas Law? If so, is this considered sufficiently realistic to make this code a viable candidate for use?

FLAC models conductive heat transport only, and does not consider convective heat transport. Despite this, it is a viable candidate for many geothermal modeling applications because it does a better job of integrating flow and geomechanics than many other codes. Density changes in the fluid are based on changes in temperature due to conductive heat transport in the rock matrix. To clarify this, we have reworded the first paragraph under 5.2.2 : "FLAC solves isothermal flow through porous media according to Darcy's Law. Convective heat transport is not considered, but fluid density can be coupled to the local temperature field. Flow equations are solved by a variant of the point-relaxation technique."

p 5.9 The discussion of MAFIC describes the use of a pre-conditioned conjugate gradient solver. So what? There are cases in which the solver used impacts the code itself (due to 
stability concerns, or requirements for damping the solution), but other than that, why make any reference to the solver. This also comes up in various of the other code discussions.

We feel that the solver used affects both the efficiency of the algorithm and the ability of the algorithm to deal with non-linear and coupled process effects, and is therefore germane to our discussions. However, we have removed the second paragraph under 5.3.2 in response to this comment.

p 5.12 2nd paragraph, what is a porous return medium? Also, 3rd paragraph; if FTRANS does not consider conservation of energy (and two phase flow) is it really a candidate code? Given a prioritized list of requirements, maybe not.

The 2nd paragraph contains a typo, and has been corrected to read "fractured porous medium." We agree that the lack of heat transport modeling capabilities is a limitation for many geothermal applications. However, because FTRANS combines fractured and porous media flow with solute transport and elastic deformation (through STEFAN), FTRANS remains a candidate code for some EGS applications. We have tried to make the limitations and applicability of FTRANS to geothermal problems clear in Section 5.4.6.

p 5.18 Section 5.6.3, solving the equations of conservation of mass and energy in an alternating sequence: does this mean the equations are decoupled? The meaning here is unclear.

To clarify this, we have modified this paragraph as follows: "MAGNUM2D solves heat flow using the same finite element solver implemented for mass flow. The coupled equations of heat flow and mass flow are solved by iteration." 
TELEPHONE: $(510)$ 527-9876

FAX: (510) 527-8164

E-MAIL: MW@ QEOTHERMEX.COM

p 5.23 Last line refers to the inability to model a moving phreatic surface. Is this important to our needs? This is the first instance it has been referred to. If it is important, perhaps it should be discussed in more detail earlier in the document.

To clarify this, we have modified the final sentence on the page to read: "NAPSAC is a singlephase (water-only) flow code."

p 5.28 Several observations. First, reference is made to standard texts that describe conservation of mass. Why not just delete? Also, assumptions used in SWIFT are given, but not given for others; make consistent. p 5.29 mention is made that SWIFT98 can be solved on PCs. If we assume this is important (though not discussed elsewhere), can any of the others? Of course, the answer is yes.

We have simplified the discussion of SWIFT by removing the first two paragraphs of page 5-28 and eliminating the bulleted list of assumptions on page 5-28. Regarding text on page 5-29, we have replaced the text "that can be solved on PCs" with "for more efficient solution."

p 6.I5 TRACR3D is not used at the INEEL.

We have modified the text accordingly.

p 7.3-7.4, Needs e-f. The treatment here is inconsistent with the balance of the discussions. The question is whether or not the simulators discussed have this feature; it is not a discussion of the feature.

We agree, and have modified the text accordingly, including some discussion on the availability of mathematical models to accommodate the features, and incorporating them into some of the codes reviewed. 